\title{
Geometric flows in Hořava-Lifshitz gravity
}

\author{
Ioannis Bakas, ${ }^{a}$ François Bourliot, ${ }^{b}$ Dieter Lüst ${ }^{c, d}$ and Marios Petropoulos ${ }^{b}$ \\ ${ }^{a}$ Department of Physics, University of Patras, \\ 26500 Patras, Greece \\ ${ }^{b}$ Centre de Physique Théorique, Ecole Polytechnique, CNRS UMR 7644, \\ 91128 Palaiseau Cedex, France \\ ${ }^{c}$ Max-Planck-Institut für Physik, \\ Föhringer Ring 6, 80805 München, Germany \\ ${ }^{d}$ Arnold-Sommerfeld-Center für Theoretische Physik, \\ Department für Physik, Ludwig-Maximilians-Universität München \\ Theresienstraße 37, 80333 München, Germany \\ E-mail: bakas@ajax.physics.upatras.gr, \\ bourliot@cpht.polytechnique.fr, dieter.luest@lmu.de, \\ marios@cpht.polytechnique.fr
}

ABSTRACT: We consider instanton solutions of Euclidean Hořava-Lifshitz gravity in four dimensions satisfying the detailed balance condition. They are described by geometric flows in three dimensions driven by certain combinations of the Cotton and Ricci tensors as well as the cosmological-constant term. The deformation curvature terms can have competing behavior leading to a variety of fixed points. The instantons interpolate between any two fixed points, which are vacua of topologically massive gravity with $\Lambda>0$, and their action is finite. Special emphasis is placed on configurations with $\mathrm{SU}(2)$ isometry associated with homogeneous but generally non-isotropic Bianchi IX model geometries. In this case, the combined Ricci-Cotton flow reduces to an autonomous system of ordinary differential equations whose properties are studied in detail for different couplings. The occurrence and stability of isotropic and anisotropic fixed points are investigated analytically and some exact solutions are obtained. The corresponding instantons are classified and they are all globally $\mathbb{R} \times S^{3}$ and complete spaces. Generalizations to higher-dimensional gravities are also briefly discussed.

Keywords: Solitons Monopoles and Instantons, Differential and Algebraic Geometry, Classical Theories of Gravity

ARXIV EPRINT: 1002.0062 


\section{Contents}

$\begin{array}{llr}1 & \text { Introduction } & 2\end{array}$

2 Non-relativistic gravity, detailed balance and flows 5

2.1 Non-relativistic gravity: a reminder 5

2.2 Euclidean action and flow equations 9

$\begin{array}{ll}2.3 & \text { Entropy functional and action bound } \\ \end{array}$

3 Bianchi IX model geometry 14

$\begin{array}{ll}3.1 \text { Some basic facts } & 14\end{array}$

$\begin{array}{lll}3.2 & \text { Ricci flow } & 15\end{array}$

$\begin{array}{lll}3.3 & \text { Cotton flow } & 17\end{array}$

4 Normalized Ricci-Cotton flow $\quad 19$

$\begin{array}{ll}\text { 4.1 The general system of Bianchi IX equations } & 19\end{array}$

$\begin{array}{lll}4.2 & \text { Classification of the fixed points } & 21\end{array}$

$\begin{array}{ll}4.3 & \text { Phase portraits of the flow } \\ 4 & 25\end{array}$

$\begin{array}{lll}4.4 & \text { Axisymmetric solutions } & 28\end{array}$

5 Ricci-Cotton flow with general couplings $\quad 31$

5.1 The axisymmetric Bianchi IX model 31

$\begin{array}{ll}5.2 & \text { Classification of the fixed points } \\ 5.32\end{array}$

$\begin{array}{lll}5.3 & \text { Phase portraits of the flow } & 35\end{array}$

$\begin{array}{lll}5.4 & \text { The special case } \Lambda_{W}=0 & 35\end{array}$

$6 \quad$ Space-time interpretation of the flow lines $\quad 39$

6.1 Global structure and completeness of the metrics 40

6.2 The action and moduli of SU(2) instanton metrics 44

7 Generalization to higher dimensions $\quad 49$

$\begin{array}{lll}7.1 & \text { Hořava-Lifshitz gravity in } 4+1 \text { dimensions } & 49\end{array}$

$\begin{array}{lll}7.2 & \text { Bach flow and its variants } & 50\end{array}$

8 Conclusions and discussion $\quad 51$ 


\section{Introduction}

Based on ideas that were originally developed in condensed matter physics [1] and later applied to the description of aspects of particle interactions [2-5], a modification of general relativity was recently proposed in $[6,7]$ and further studied under the name of HoravaLifshitz gravity. In this theory, which includes higher-order curvature terms on spatial slices, the diffeomorphism invariance of general relativity is broken explicitly setting a privileged time direction. This affects the ultra-violet behavior of the quantum theory, which, hence, looks power-counting renormalizable. Some efforts have been made to prove consistency of the quantum theory $[8,9]$, but the number of propagating degrees of freedom seems to invalidate the matching with Einstein's gravity in the infrared regime, and hence seems to disprove this theory as a viable alternative to general relativity [10-15]. The investigation of these issues is still going on. Despite the difficulties and reservations, the Hořava-Lifshitz gravity still provides an interesting classical and quantum field theory framework, where one can address some interesting questions and explore several connections to ordinary gravity or string theory. These also include the appearance and relevance of geometric flows, which is the main subject of the present work.

Geometric flows, and, in particular, Ricci flows are interesting in their own right. In mathematics they turned out to play a crucial role in implementing Hamilton's program for proving Poincaré's and Thurston's conjectures [16-19] (but see also [20] and [21] and references therein). In physics they originally appeared in off-critical string theory via the renormalization-group equations of two-dimensional non-linear sigma models, where the evolution of the metric under the Ricci flow equations provides the running of the bulk coupling to lowest order in perturbation theory (see $[22,23]$ for the original result). In this context, the renormalization-group time is provided by the logarithmic length scale of the world-sheet, but in some cases it can also assume the role of genuine time, describing realtime evolution in string theory in regimes where the friction due to the motion of the dilaton effectively reduces the second-order evolution equations to the first-order renormalizationgroup flow equations [24, 25].

Ricci flow models also appear in the framework of four-dimensional gravitational instantons of general relativity. Solving Einstein's equations is, in general, an impossible task. It is substantially simplified under the assumption of self-duality as a sufficient condition to find vacuum solutions in the Euclidean sector of the theory. Homogeneity of spatial sections is often a further simplification to find explicit solutions. Although what we call space is somewhat arbitrary in Euclidean gravity, the latter statement can be made precise by assuming a foliation in three-dimensional leaves that are invariant under an isometry group of motions. For these particular vacuum solutions, it turns out that the Euclidean time evolution of the homogeneous leaves inside the gravitational instanton can be recast as Ricci flow equations for the corresponding geometry on the homogeneous model spaces [25-29].

The modification of gravity proposed by Hořava in $[6,7]$ shares some features with the previous setting that allow to define the analogue of gravitational-instanton configurations. In particular, a foliation of the four-dimensional space is assumed from the very beginning 
with a privileged time direction at the level of the action. Furthermore, a condition called detailed balance, which is borrowed from non-equilibrium thermodynamics, requires that the dynamics follows from an appropriately chosen three-dimensional superpotential action. In the Euclidean version of the theory, this resembles the self-duality condition with similar consequences: for a class of configurations that minimize the action, the time evolution becomes first-order and describes a geometric flow on the leaves of the foliation. Unlike general relativity, where Ricci flow is equivalent to self-duality only for configurations with homogeneous leaves, the description of instanton-like solutions by geometric flows is generic in Hořava-Lifshitz gravity. The nature of the corresponding flow depends on the choice of the three-dimensional action used for detailed balance and it is driven, in general, by a certain combination of Ricci and Cotton tensors as well as the cosmological constant term. It is not our concern, and it will not be pursued here at all, to find whether such combinations of curvature tensors can also arise from the renormalization- group equations of some quantum field theory.

Our aim in this paper is to investigate aspects of the Euclidean dynamics of HoravaLifshitz gravity with detailed balance. Our motivations are diverse. First, classical instantonlike solutions are important for the determination of transition amplitudes in quantum gravity. They are also useful in the Hartle-Hawking formulation of quantum cosmology [30], even though classical cosmology per se requires the analysis of real-time equations. The geometric flows that emerge in this framework involve tensors with higher-order spatial derivatives terms, and, as such, they are new in the literature; they reduce to previously studied examples only for some special values of their parameters. Thus, it is instructive to formulate the flow equations in all generality, determine the nature of the fixed points and their stability properties, obtain explicit solutions, as well as study general questions such as the monotonicity of the evolution, the possible formation of singularities, the occurrence of bounces and so on. These questions arise naturally in the general theory of geometric flows and they are bound to be relevant for the space-time interpretation of the analogue of gravitational instantons in Hořava-Lifshitz theory.

The answer to these questions will be accomplished partially using some ansatz for the underlying three-dimensional spaces, leading to mini-superspace truncation of the flow equations. Otherwise, it does not be seem possible to draw general conclusions for the general system of equations, at least at the current level of our understanding of this problem. Still, the results that will be described are indicative of what should be expected in general. More systematic investigation of the infinite-dimensional dynamical system at hand requires substantial mathematical work that is not contained in this paper. Following the paradigm of gravitational instantons with isometry groups in Euclidean Einstein gravity, we will consider homogeneous geometries on the three-dimensional spatial slices of HoravaLifshitz gravity and focus, in particular, to the case of Bianchi IX geometry as a class of homogeneous but generally non-isotropic deformation of $S^{3}$; this model is often referred to as mixmaster universe in the Lorentzian (real-time) approach to cosmology [31-35], and was recently discussed in the framework of Hořava-Lifshitz gravity [36, 37]. Other Bianchi classes as well as more general inhomogeneous deformations of the three-sphere (under appropriate ansatz) can also be studied along similar lines, but they will not be discussed. 
We set up the general problem using the Bianchi IX model geometry and study in detail some specific examples of the flow for different couplings in Hořava-Lifshitz gravity. Even in this case the resulting equations in mini-superspace are not easily tractable for generic values of the couplings. First, we will consider the Ricci flow and some of its variants that describe solutions of the modified theory of gravity with anisotropy scaling parameter $z=2$ (see next section for this and other definitions) and compare them to instantons with $\mathrm{SU}(2)$ isometry in ordinary gravity. We will also consider the Cotton flow, separately, and use it to construct solutions of Hořava-Lifshitz theory with anisotropy scaling parameter $z=3$ by ignoring all Ricci curvature terms that become subdominant when the volume of $S^{3}$ is very small. We will also consider the combined Ricci-Cotton flow and explore the equations in detail first in the limit that the speed of light vanishes or equivalently Newton's constant becomes infinite (it is often called Carroll limit after [38]). The normalized Ricci-Cotton flow and the unnormalized variant of it with vanishing cosmological constant provide the relevant equations in this limit. Finally, we will consider the general Ricci-Cotton flow with arbitrary couplings and obtain several qualitative results for its solutions.

In all cases it is assumed that the parameter of the superspace metric of the theory is restricted to values $\lambda<1 / 3$ (in which case the cosmological constant will also be taken non-negative) so that the flow equations extremize the classical action, up to important boundary terms. Proper account of the boundary terms leads to the definition of instanton solutions as finite-action trajectories that interpolate between fixed points. Our analysis shows that the Ricci and the Cotton tensor terms can compete with each other, and, depending on the relative sign between the two, the flow equations can exhibit symmetric as well as anisotropic fixed points. The nature of these fixed points and their stability properties also have implications for the space-time interpretation of the corresponding gravitational instanton solutions. Axisymmetric solutions are associated with spaces with $\mathrm{SU}(2) \times \mathrm{U}(1)$ isometries, and, hence they are easier to describe in closed form.

Instanton solutions of Hořava-Lifshitz gravity, as they are defined, are rather special configurations that rely on the existence of multiple-degenerate vacua and correspond to special flow lines, which guarantee finiteness of their action. As it will turn out, they are also free of singularities and their space-time metrics are regular and complete. Note, however, that other flow lines, possibly with infinite action, also describe solutions of the secondorder equations of motion, but they may have singularities. Although we are primarily interested in the class of instanton solutions, one should be open-minded for other more general possibilities too. For this reason, as well as for mathematical completeness, we will investigate the phase portraits of the flow equations in all generality. The selection of special trajectories that correspond to instantons will be made much later together with their space-time interpretation.

In section 2, we first briefly review the formulation of Hořava-Lifshitz gravity with detailed balance condition putting emphasis on the structure of the potential term and its associated superpotential. This analysis is then carried to the Euclidean regime where "zero-energy" (i.e., self-dual-like) configurations exist satisfying the flow equations. Restrictions on the parameter $\lambda$ are also obtained together with an entropy functional that changes monotonically along the flow lines. The results are then used to define instan- 
ton solutions as in ordinary point particle systems. The Bianchi IX model geometry is introduced in section 3 where the truncation of the Ricci and Cotton flows are studied separately in detail. Section 4 is entirely devoted to the analysis of the normalized RicciCotton flow and the explicit construction of its axisymmetric solutions. Section 5 discusses the case of unnormalized Ricci-Cotton flow obtained for general couplings. It contains the case study of positive and zero cosmological constant for axisymmetric configurations with $\lambda<1 / 3$. Section 6 is devoted to the space-time interpretation of the flow line, as gravitational instantons, making comparisons with the analogous instanton solutions arising in general relativity. Complete classification of all gravitational-instanton metrics with $\mathrm{SU}(2)$ isometry is also obtained. In section 7 we outline generalizations of the framework to higher-dimensional Hořava-Lifshitz gravities, which, for instance, in $4+1$ dimensions give rise to a new system of flow equations on four-manifolds driven by the Bach tensor. Finally, section 8 contains our conclusions and poses several questions for future work.

\section{Non-relativistic gravity, detailed balance and flows}

\subsection{Non-relativistic gravity: a reminder}

The theory of non-relativistic gravity developed in $[6,7]$ is valid for general space-time dimension $D+1$. It has three main features:

- Space-time is assumed to be topologically $\mathcal{M}_{D+1}=\mathbb{R} \times \mathcal{M}_{D}$, leading to a natural codimension-one foliation. Diffeormorphism invariance is broken down to the subgroup of foliation-preserving transformations. This breaking is controlled by a parameter $\lambda$.

- Scaling properties of space and time are different and captured by an integer $z$. Power counting renormalizability of the theory requires $z=D$.

- The interactions are determined by a detailed balance condition following from a Euclidean $D$-dimensional diffeomorphism-invariant action, which gives rise to marginal and relevant terms in $D+1$ dimensions.

The last item above is not generic in Hořava-Lifshitz gravity and it can be relaxed by allowing more arbitrary coefficients for the various marginal and relevant terms. However, it is a necessary ingredient in our study to connect it naturally with the theory of geometric flows. Thus, detailed balance will be assumed in the following.

Let us adopt the ADM (Arnowitt-Deser-Misner) decomposition of the metric (see, for instance, [39]), which is suitable for the $D+1$ foliation of space-time,

$$
\mathrm{d} s^{2}=-N^{2} \mathrm{~d} t^{2}+g_{i j}\left(\mathrm{~d} x^{i}+N^{i} \mathrm{~d} t\right)\left(\mathrm{d} x^{j}+N^{j} \mathrm{~d} t\right)
$$

where $N^{i}$ and $N$ are the shift and lapse functions respectively. Here, $i, j, \ldots$ run in $D$ dimensions and all tensors that will appear in the following are $D$-dimensional. 
Using this decomposition, the Einstein-Hilbert action in $D+1$ dimensions (up to total derivative terms that may contribute in topologically non-trivial spaces) reads as follows,

$$
S_{\mathrm{EH}}=\frac{1}{16 \pi G_{\mathrm{N}}} \int \mathrm{d}^{D+1} x \sqrt{g} N\left(K_{i j} K^{i j}-K^{2}+R-2 \Lambda\right),
$$

where $\Lambda$ is the genuine cosmological constant in $D+1$ dimensions. In this expression, $K_{i j}$ is the second fundamental form that measures the extrinsic curvature of the leaves at constant $t$,

$$
K_{i j}=\frac{1}{2 N}\left(\partial_{t} g_{i j}-\nabla_{i} N_{j}-\nabla_{j} N_{i}\right)
$$

and its trace $K=g^{i j} K_{i j}$ is the mean curvature. The first two terms in equation (2.2) provide the kinetic energy, since they include time derivatives of the field $g_{i j}$. Their specific combination can be recast in the form

$$
K_{i j} G_{\mathrm{DW}}^{i j k \ell} K_{k \ell}=K_{i j} K^{i j}-K^{2}
$$

using the DeWitt metric in superspace

$$
G_{\mathrm{DW}}^{i j k \ell}=\frac{1}{2}\left(g^{i k} g^{j \ell}+g^{i \ell} g^{j k}\right)-g^{i j} g^{k \ell}
$$

The potential term in Einstein gravity is provided by the three-dimensional Ricci scalar curvature $R$ and the four-dimensional cosmological constant term $\Lambda$ (when it is present), as shown in (2.2).

In non-relativistic gravity, space and time scale as $[t]=-z,[x]=-1$ and it is further assumed $^{1}$ that $\left[N_{i}\right]=z-1,[N]=0$ and $\left[g_{i j}\right]=0$ so that $\left[K^{2}\right]=2 z$; it should be contrasted to general relativity where space and time scale the same with $z=1$. This asymmetry is further implemented in the action, both in the kinetic and the potential terms by requiring foliation preserving covariance. The kinetic term is generalized as

$$
S_{\mathrm{K}}=\frac{2}{\kappa^{2}} \int \mathrm{d} t \mathrm{~d}^{D} x \sqrt{g} N K_{i j} G^{i j k \ell} K_{k \ell}=\frac{2}{\kappa^{2}} \int \mathrm{d} t \mathrm{~d}^{D} x \sqrt{g} N\left(K_{i j} K^{i j}-\lambda K^{2}\right),
$$

where $\left[\kappa^{2}\right]=z-D$ and $\lambda$ is a dimensionless coupling measuring the breaking of the full diffeomorphism group. Here, $G^{i j k \ell}$ is the generalized metric in superspace

$$
G^{i j k \ell}=\frac{1}{2}\left(g^{i k} g^{j \ell}+g^{i \ell} g^{j k}\right)-\lambda g^{i j} g^{k \ell} .
$$

that coincides with the DeWitt metric when $\lambda=1$. It is worth stressing that this metric can be positive-definite or indefinite depending on $\lambda$. Indeed, $g_{k \ell}$ is an "eigenvector",

$$
G^{i j k \ell} g_{k \ell}=(1-\lambda D) g^{i j}
$$

with eigenvalue $1-\lambda D$. The sign of the latter changes at $\lambda=1 / D$ where the inverse no longer exists. Thus, for $\lambda<1 / D$ the metric is positive-definite and it becomes indefinite

\footnotetext{
${ }^{1}$ Proper restoration of the speed of light, which scales as $[c]=z-1$, explains the various dimensions, as described in detail in the original works $[6,7]$.
} 
for all $\lambda>1 / D$ that include, in particular, $\lambda=1$. This behavior and the fact that $\lambda$ is ultimately an unprotected parameter of the theory, which, in principle can take any real value, should be kept in mind when considering quantum corrections.

The potential term of the theory has the general form

$$
S_{V}=-\int \mathrm{d} t \mathrm{~d}^{D} x \sqrt{g} N V[g]
$$

and can also contribute in various ways to the breaking of diffeomorphism invariance. Note that $[V]=z+D$ and there is a large freedom to choose $V$ so that it includes operators of dimension less than or equal to $z+D$ (called relevant and marginal operators, respectively). In order to reduce this freedom and take advantage of the renormalization properties of a $D$-dimensional system, it was proposed in $[6,7]$ to introduce a detailed balance condition that allows to express the potential in terms of a "superpotential" as follows:

$$
V=\frac{\kappa^{2}}{2} E^{i j} \mathcal{G}_{i j k \ell} E^{k \ell}
$$

where

$$
E^{i j}=-\frac{1}{2 \sqrt{g}} \frac{\delta W[g]}{\delta g_{i j}}
$$

and $W$ a $D$-dimensional action so that $\left[E^{i j}\right]=D$. The tensor $\mathcal{G}_{i j k \ell}$ is defined as

$$
\mathcal{G}_{i j k \ell}=\frac{1}{2}\left(g_{i k} g_{j \ell}+g_{i \ell} g_{j k}\right)-\frac{\lambda}{D \lambda-1} g_{i j} g_{k \ell}
$$

and coincides with the inverse of the metric in superspace with generic $\lambda$, i.e.,

$$
G^{i j k \ell} \mathcal{G}_{k \ell m n}=\frac{1}{2}\left(\delta_{m}^{i} \delta_{n}^{j}+\delta_{n}^{i} \delta_{m}^{j}\right)
$$

The resulting theory is not invariant under general coordinate transformations of spacetime. Indeed, since $\mathcal{M}_{D+1}$ is topologically $\mathbb{R} \times \mathcal{M}_{D}$, it is only appropriate to consider invariance of the action under the restricted class of foliation-preserving diffeomorphisms,

$$
\tilde{t}=\tilde{t}(t), \quad \tilde{x}^{i}=\tilde{x}^{i}(t, x) .
$$

Then, the lapse function $N$ associated with the freedom of time reparametrization is restricted to be a function of $t$ alone, whereas the shift functions $N_{i}$ associated with diffeomorphisms of $\mathcal{M}_{D}$ can depend on all space-time coordinates. This is often called the projectable case of Hořava-Lifshitz gravity and it will be assumed in the following. The non-projectable version of the theory leads to dynamical inconsistencies [40].

The choice of $W$ depends on the dimension $D$. Here, we recall the choice for $D=3$ with $z=3$ that ensures power-counting renormalizability of the four-dimensional theory; generalization to higher dimensions will be discussed later in section 7 . Then, the marginal operators in question are obtained from the three-dimensional gravitational Chern-Simons action, which is familiar from topologically massive gravity [41, 42],

$$
W_{\mathrm{CS}}=\frac{1}{w_{\mathrm{CS}}} \int \omega_{3}(\omega),
$$


with density given in terms of the connection one-form $\omega$ by

$$
\omega_{3}(\omega)=\frac{1}{2} \operatorname{Tr}\left(\omega \wedge \mathrm{d} \omega+\frac{2}{3} \omega \wedge \omega \wedge \omega\right) .
$$

The corresponding variation gives

$$
E_{\mathrm{CS}}^{k \ell}=-\frac{1}{w_{\mathrm{CS}}} \frac{\varepsilon^{i j k}}{\sqrt{g}} \nabla_{i}\left(R_{j}^{\ell}-\frac{1}{4} R \delta_{j}^{\ell}\right) \equiv-\frac{1}{w_{\mathrm{CS}}} C^{k \ell},
$$

where $C^{k \ell}$ is the Cotton tensor and $\epsilon^{123}=1$. The Cotton tensor is traceless, conserved and it vanishes identically for conformally flat metrics.

Relevant operators in four dimensions are generated by the Einstein-Hilbert threedimensional action

$$
W_{\mathrm{EH}}=\frac{2}{\kappa_{W}^{2}} \int \mathrm{d}^{D} x \sqrt{g}\left(R-2 \Lambda_{W}\right) .
$$

Note that neither $\kappa_{W}^{2}$ is the four-dimensional Newton's constant nor $\Lambda_{W}$ is the fourdimensional cosmological constant, but they will be identified shortly. The variation of this action leads to

$$
E_{\mathrm{EH}}^{k \ell}=\frac{1}{\kappa_{W}^{2}}\left(R^{k \ell}-\frac{R}{2} g^{k \ell}+\Lambda_{W} g^{k \ell}\right) .
$$

Combining the Chern-Simons and Einstein-Hilbert contributions to $E^{k \ell}$, with their respective couplings, the full potential of Hořava-Lifshitz gravity reads

$$
V=\frac{\kappa^{2}}{2 w_{\mathrm{CS}}^{2}} C^{i j} C_{i j}-\frac{\kappa^{2}}{w_{\mathrm{CS}} \kappa_{W}^{2}} C^{i j} R_{i j}+\frac{\kappa^{2}}{2 \kappa_{W}^{4}}\left(R^{i j} R_{i j}-\frac{4 \lambda-1}{4(3 \lambda-1)} R^{2}\right)+\frac{\kappa^{2} \Lambda_{W}}{2(3 \lambda-1) \kappa_{W}^{4}}\left(R-3 \Lambda_{W}\right) .
$$

The ultra-violet behavior of the resulting theory is dictated by the quadratic Cotton curvature term, which is marginal with dimension $2 z=6$, and corresponds to $z=3$. It improves a lot the ultra-violet behavior of ordinary Einstein gravity at the expense of breaking general covariance of the theory at short distances. When the Cotton term is absent, the resulting theory has a potential with quadratic Ricci curvature terms that become dominant in the ultra-violet regime and so $z=2$. In either case, in the infrared limit one expects to flow by the most relevant operators (of dimension 2 and zero), which correspond to the last terms in equation (2.20), and recover general relativity provided that $\lambda$ also flows to 1 . However, no rigorous proof of any of these statements is yet available in the literature. Also, the counting of physical degrees of freedom of the theory, which is crucial for viewing it as viable modification of general relativity, is obscured by the outcome of local invariances and their potential restoration.

We also recall for completeness, using the relativistic coordinate $x^{0}=c t$, that the effective speed of light for general $\lambda$ is given by

$$
c=\frac{\kappa^{2}}{2 \kappa_{W}^{2}} \sqrt{\frac{\Lambda_{W}}{1-3 \lambda}}
$$

with $[c]=z-1$. This shows that $\Lambda_{W}$ must be negative when $\lambda>1 / 3$ to ensure reality of $c$; likewise, $\Lambda_{W}$ must be positive when $\lambda<1 / 3$. Also, the four-dimensional effective 
cosmological constant is given by

$$
\Lambda=\frac{3}{2} \Lambda_{W}
$$

and, therefore, the range $\lambda>1 / 3$ does not allow for de Sitter-like backgrounds in HořavaLifshitz gravity. These identifications are necessary in order to compare the infrared limit of the deformed theory to ordinary gravity so that the effective Newton constant reads as

$$
G_{N}=\frac{\kappa^{2}}{32 \pi c} .
$$

Furthermore, $\lambda$ should approach (flow to) 1 in the infrared limit in order to recover the full reparametrization invariance of general relativity, but this particular problem will not concern us at all here.

The search for classical solutions requires the use of the potential (2.20) and it is impossible to solve in full generality. Symmetry ansatz such as spatial homogeneity makes the problem more tractable, but still not exactly solvable. This includes, for example, the case of Bianchi IX geometry leading to the mixmaster universe model in four space-time dimensions with Lorentzian signature. We will not pursue this line of investigation here (see [36] for a detailed analysis and comparison with the mixmaster universe in general relativity [31-34]), but elaborate on the Euclidean version of Hořava-Lifshitz gravity and then analyze its instanton solutions for Bianchi IX spatial geometries.

\subsection{Euclidean action and flow equations}

Besides the various physical motivations pertaining to the analysis of the Euclidean version of Hořava-Lifshitz theory, there is also a technical advantage for constructing solutions that satisfy first-order equations in time. This possibility is also encountered in general relativity when self-duality on the Riemann (and more generally on the Weyl) tensor is imposed leading to gravitational-instanton solutions in the Euclidean regime. ${ }^{2}$ Although there is no direct analogue of self-duality in gravitational theories with anisotropic scaling, the detailed balance condition offers the appropriate replacement for defining instanton-like configurations. This is in fact possible in all dimensions unlike gravitational instantons of ordinary gravity that are only defined in four space-time dimensions.

The Euclidean action is obtained by setting $t \rightarrow-i t, N^{j} \rightarrow i N^{j}$, whereas $i S$ is traded for $-S$. Using equations (2.6) and (2.9) one obtains

$$
S=\int \mathrm{d} t \mathrm{~d}^{D} x \sqrt{g} N\left(\frac{2}{\kappa^{2}}\left(K_{i j} K^{i j}-\lambda K^{2}\right)+V\right) .
$$

The expression for the potential (2.10) allows to rewrite the Euclidean action (2.24) in the form $S=S^{\prime}+S^{\prime \prime}$, where

$$
S^{\prime}=\frac{2}{\kappa^{2}} \int \mathrm{d} t \mathrm{~d}^{D} x \sqrt{g} N\left(K_{i j} \pm \frac{\kappa^{2}}{2} \mathcal{G}_{i j m n} E^{m n}\right) G^{i j k \ell}\left(K_{k \ell} \pm \frac{\kappa^{2}}{2} \mathcal{G}_{k \ell r s} E^{r s}\right)
$$

\footnotetext{
${ }^{2}$ Self-duality is best described in terms of the curvature two-form, as $\mathcal{R}_{a b}= \pm \tilde{\mathcal{R}}_{a b}$ in an orthonormal frame [43]. These equations are second-order in time, but they can be integrated once to yield firstorder equations [44] that will be paralleled to the instanton solutions of Hor̆ava-Lifshitz gravity; for further details see also [29], where this analogy is made even sharper for gravitational instantons with homogeneous spatial sections.
} 
and $S^{\prime \prime}$ is a total-derivative contribution to the action [6]. This boundary term will be considered later in detail (see equation (2.33)). The different signs correspond to the choice of time direction.

The action (2.25) is bounded below by zero provided that the superspace metric $G^{i j k \ell}$ is positive-definite. Then, configurations that obey the first-order differential equations

$$
K_{i j}=\mp \frac{\kappa^{2}}{2} \mathcal{G}_{i j k \ell} E^{k \ell}
$$

are extrema of the action and as such they provide solutions of the Euclidean theory $S^{\prime}$; the leaves of the corresponding space-time foliations have prescribed extrinsic curvature. This possibility arises only when $\lambda<1 / D$, in which case we must also demand that $\Lambda_{W}$ is nonnegative so that the speed of light in the Lorentzian version of the theory is real. Otherwise, for $\lambda>1 / D$, the action is non-bounded below by zero and the first-order equations are not guaranteed to provide classical solutions. Thus, from now on, we restrict ourselves to $\lambda<1 / D$ and $\Lambda_{W} \geq 0$, where the first-order equations (2.26) provide extrema of $S^{\prime}$. They are also extrema of the action $S$ provided that the boundary term $S^{\prime \prime}$ is properly accounted. This problem will be treated carefully in section 2.3 and lead to the precise definition of instantons.

The solutions that we will investigate can be expressed in the form of geometric gradient flow equations for the metric $g_{i j}$ modulo reparametrizations generated by the shift functions,

$$
\partial_{t} g_{i j}=\mp \kappa^{2} N \mathcal{G}_{i j k \ell} E^{k \ell}+\nabla_{i} N_{j}+\nabla_{j} N_{i}
$$

Since we are only considering the projectable case of Hořava-Lifshitz gravity, $N$ is only a function of $t$ and can be absorbed by redefining time, as $N(t) \mathrm{d} t \rightarrow \mathrm{d} t$. It is also natural to define vector fields with components $\xi_{i}=N_{i} / N$ that generally depend on space and time coordinates. Then, the geometric-flow equations assume the more standard form that will be used in the following,

$$
\frac{1}{N(t)} \partial_{t} g_{i j}= \pm \frac{\kappa^{2}}{2 \sqrt{g}} \mathcal{G}_{i j k \ell} \frac{\delta W[g]}{\delta g_{k \ell}}+\nabla_{i} \xi_{j}+\nabla_{j} \xi_{i}
$$

Specializing to $D=3$, we write down explicitly the flow equations obtained by combining the variation of the Chern-Simons and Einstein-Hilbert actions,

$$
\frac{1}{N} \partial_{t} g_{i j}=-\frac{\kappa^{2}}{\kappa_{W}^{2}}\left(R_{i j}-\frac{2 \lambda-1}{2(3 \lambda-1)} R g_{i j}+\frac{\Lambda_{W}}{1-3 \lambda} g_{i j}\right)+\frac{\kappa^{2}}{w_{\mathrm{CS}}} C_{i j}+\nabla_{i} \xi_{j}+\nabla_{j} \xi_{i}
$$

choosing for definiteness one of the two sign options; the other follows by time reversal. These equations describe the parametric evolution of a family of three-dimensional geometries

$$
\mathrm{d} s_{\mathrm{t}}^{2}=g_{i j}(x ; t) \mathrm{d} x^{i} \mathrm{~d} x^{j}
$$


driven by the Ricci and Cotton tensors and the cosmological constant term, and, as such, they will be called Ricci-Cotton flow equations. ${ }^{3}$

The fixed points are determined (modulo reparametrization terms) by the solutions of three-dimensional topologically massive gravity:

$$
\frac{\kappa_{W}^{2}}{w_{\mathrm{C} S}} C_{i j}=R_{i j}-2 \Lambda_{W} g_{i j}, \quad \text { with } R=6 \Lambda_{W} .
$$

They include Einstein metrics with vanishing Cotton tensor, like the round sphere metric on $S^{3}$ (for $\Lambda_{W}>0$ ), which is homogeneous and isotropic. There are other fixed points, however, with constant scalar curvature but with non-vanishing Cotton tensor. We will see later, as example, that they correspond to particular homogeneous but non-isotropic metrics on $S^{3}$. The coexistence of fixed points from different conformal classes of the metric make this flow particularly complex.

The driving terms of the Ricci-Cotton flow involve, in general, third-order derivatives in space coordinates (originating from the Cotton tensor), and, therefore, it is not possible to apply standard results from the mathematics literature to prove even the short-time existence of solutions. Nevertheless, the mini-superspace models that will be studied later show that these flow equations are well-behaved and the trajectories converge to fixed points after sufficiently long time.

Some special cases are worth noting, since they have already appeared in the literature for different reasons:

- $w_{\mathrm{C} S} \rightarrow \infty$ : the Cotton tensor contribution drops out and one obtains a variant of the Ricci flow on three-manifolds, which is second-order and well studied in the literature. Its trajectories describe solutions of $z=2$ Hořava-Lifshitz gravity in $3+1$ dimensions, whereas the fixed points are Einstein metrics $R_{i j}=2 \Lambda_{W} g_{i j}$.

- $\kappa_{W}^{2} \rightarrow \infty$ : the Ricci and cosmological constant terms drop out and one obtains the pure Cotton flow that was recently introduced in the literature [46]. Its trajectories describe solutions of $z=3$ Hořava-Lifshitz gravity in $3+1$ dimensions, in the limit under consideration. The fixed points are conformally flat metrics, $C_{i j}=0$.

Even these simpler cases are impossible to solve in all generality. Mini-superspace models have been used to study the long time behavior of the Ricci and Cotton flows for homogeneous geometries [46, 47].

Apart from the Ricci and Cotton flows that will studied separately in the next section, there are also some other special cases of the combined Ricci-Cotton flow that are relatively easier to study. First, by considering that limiting case $\lambda \rightarrow-\infty$, which lies in the allowed range $\lambda<1 / 3$, one obtains the normalized Ricci-Cotton flow, which is driven by a traceless tensor, and, thus, preserves the volume of space; the cosmological constant decouples in

\footnotetext{
${ }^{3}$ Perhaps a more appropriate name is Ricci-Yamabe-Cotton flow, since $R g_{i j}$ is the driving term of the so called Yamabe flow, $\partial_{t} g_{i j}=-R g_{i j}$. The latter was introduced in the literature [45] to solve Yamabe's conjecture stating that any metric is conformally equivalent to a metric of constant curvature. Its effect is complementary to the Cotton term of the flow, which changes the conformal class of the metric. Thus, the combined flow equations we have obtained contain several competing deformations of the metric.
} 
this case. It becomes relevant in the Carroll limit of Hořava-Lifshitz gravity, where the effective speed of light vanishes. ${ }^{4}$ The effective speed of light vanishes also when $\Lambda_{W}=0$, irrespective of $\lambda$, and the corresponding flow equations will be studied separately. The general case, with arbitrary coefficients, is much more complex. The pattern of fixed points and specific trajectories will only be discussed for axially symmetric deformations of $S^{3}$, which correspond to solutions with $\mathrm{SU}(2) \times \mathrm{U}(1)$ isometry.

\subsection{Entropy functional and action bound}

From now on, and in all examples that will be studied later, we consider flows without the effect of space reparametrizations, setting $N_{i}=0$. Also, we will take advantage of time reparametrizations to set $N(t)=1$ for convenience. We will also assume that the spatial slices are compact spaces without boundary. Here, we provide an entropy functional for the geometric flows arising in Hořava-Lifshitz gravity in arbitrary dimensions. This functional is also be related to the lower bound of the Euclidean action $S$ (rather than $S^{\prime}$ ) when boundary terms $S^{\prime \prime}$ are properly taken into account.

When the metric in superspace is positive-definite (choosing $\lambda<1 / D$ in $D$ spatial dimensions), the superpotential functional $W$ changes monotonically along the flow. This follows easily by considering

$$
\frac{\mathrm{d} W}{\mathrm{~d} t}=-2 \int \mathrm{d}^{D} x \sqrt{g} E^{i j} \partial_{t} g_{i j}= \pm 2 \kappa^{2} \int \mathrm{d}^{D} x \sqrt{g} E^{i j} \mathcal{G}_{i j k \ell} E^{k \ell},
$$

which is the integral of a quadratic quantity, and, therefore, increases or decreases monotonically depending on the overall sign. Thus, $W$ is an entropy functional for the flows under consideration.

With this in mind, let us revisit the original Euclidean action $S$ of the theory and its lower bound taking into proper account the boundary terms. Equations (2.24) and (2.10) yield

$$
\begin{aligned}
S= & \frac{2}{\kappa^{2}} \int \mathrm{d} t \mathrm{~d}^{D} x \sqrt{g} K_{i j} G^{i j k \ell} K_{k \ell}+\frac{\kappa^{2}}{2} \int \mathrm{d} t \mathrm{~d}^{D} x \sqrt{g} E^{i j} \mathcal{G}_{i j k \ell} E^{k \ell} \\
= & \frac{2}{\kappa^{2}} \int \mathrm{d} t \mathrm{~d}^{D} x \sqrt{g}\left(K_{i j} \pm \frac{\kappa^{2}}{2} \mathcal{G}_{i j m n} E^{m n}\right) G^{i j k \ell}\left(K_{k \ell} \pm \frac{\kappa^{2}}{2} \mathcal{G}_{k \ell r s} E^{r s}\right) \\
& \mp 2 \int \mathrm{d} t \mathrm{~d}^{D} x \sqrt{g} K_{i j} E^{i j} .
\end{aligned}
$$

The first term in (2.33) is $S^{\prime}$, given in equation (2.25), and the last term is the advertised boundary contribution $S^{\prime \prime}$. For positive-definite superspace metric, the Euclidean action $S$ is bounded from below by the boundary term $S^{\prime \prime}$, because $S^{\prime} \geq 0$. Thus,

$$
S \geq \mp 2 \int \mathrm{d} t \mathrm{~d}^{D} x \sqrt{g} K_{i j} E^{i j}=\mp \int \mathrm{d} t \mathrm{~d}^{D} x \sqrt{g} E^{i j} \partial_{t} g_{i j}= \pm \frac{1}{2} \int \mathrm{d} t \frac{\mathrm{d} W}{\mathrm{~d} t},
$$

\footnotetext{
${ }^{4}$ When $\lambda \rightarrow-\infty$, the metric in superspace becomes singular as its inverse has zero eigenvalues. Yet the flow equations are well-defined and so is the potential term $S_{V}$ of the gravity action. It is opposite to the case $\lambda=1 / 3$ for which the metric in superspace has zero eigenvalues and its inverse becomes singular; the latter case corresponds to the limit of infinite speed of light, where the theory develops anisotropic Weyl invariance.
} 
having set $N(t)=1$. The time integral of equation (2.32) shows that the lower bound of $S$ is always positive, as expected.

The flow equations (2.26) provide time-dependent extrema of the action $S^{\prime}$. They are actually its ground states, since they make $S^{\prime}$ vanish. Since $S^{\prime \prime}$ is a boundary term, these ground states are also extrema of $S$ under appropriate boundary conditions that make the variational problem well-posed. This can be easily verified for the class of flows with finite action (i.e. finite $S^{\prime \prime}$ ). Note for this purpose that the fixed points of the flow are static solutions of both $S$ and $S^{\prime}$, since they are, by construction, critical points of the $D$-dimensional action functional $W$ sitting at the minima of the Hořava-Lifshitz potential. If different minima exist, they will be all degenerate with zero potential energy. Hence, time-dependent solutions that interpolate between any two fixed points are guaranteed to satisfy the equations of motion following from the Hořava-Lifshitz action $S$. These solutions have finite action, given by the value of the boundary term, and is natural to call them instantons as they interpolate between two different static minima, which are connected by trajectories of the geometric flow. Their action is simply given by

$$
S_{\text {instanton }}=\frac{1}{2}|\Delta W|,
$$

where $\Delta W$ is the difference of the corresponding values of $W$ at the two critical points. Note that $\Delta W \neq 0$, in general, since $W$ changes monotonically along the flow lines and the instanton action is finite. Then, this yields the standard description of instanton solutions of a point particle moving in Euclidean time, but the number of degrees of freedom is infinite now, as the evolution takes place in superspace.

Finally, let us consider the evolution of the volume of spatial slices under the flow. In general, it takes the form

$$
\frac{\mathrm{d}}{\mathrm{d} t} \operatorname{vol}\left(\mathcal{M}_{D}\right)=\frac{1}{2} \int \mathrm{d}^{D} x \sqrt{g} g^{i j} \partial_{t} g_{i j}=\mp \frac{\kappa^{2}}{2(1-3 \lambda)} \int \mathrm{d}^{D} x \sqrt{g} g_{i j} E^{i j}
$$

and, therefore, the trace-free part of the driving curvature terms do not contribute to the evolution. Otherwise, the volume changes without definite sign. Thus, in principle, the volume can bounce along the flow. At the fixed points, where $g_{i j} E^{i j}=0$, the volume reaches a local maximum or minimum depending on circumstances.

As an example, let us consider the Ricci-Cotton flow described by equation (2.29). Then, since the Cotton tensor is traceless, the volume of space changes as

$$
\frac{\mathrm{d}}{\mathrm{d} t} \operatorname{vol}\left(\mathcal{M}_{3}\right)=\frac{\kappa^{2}}{4(1-3 \lambda) \kappa_{W}^{2}} \int \mathrm{d}^{D} x \sqrt{g}\left(R-6 \Lambda_{W}\right)
$$

and it can have either sign. Of course, it is possible to normalize this (or any other flow that arises in this context) by rescaling the metric with a function of time followed by a suitably chosen time reparametrization $t \rightarrow \tilde{t}(t)$ so that the volume is preserved in time $\tilde{t}$. This does not resolve the problem, however, since $\tilde{t}(t)$ is not a monotonic function of $t$ in general. Thus, the volume does not provide an entropy functional.

Other entropy functionals might also exist for these flows, generalizing Perelman's functional for the Ricci flow [17-19], but we have not been able to find them. 


\section{Bianchi IX model geometry}

All homogeneous space geometries in three dimensions provide consistent ansatz for the mini-superspace truncation of the Ricci and Cotton flows and their combination thereof. Such spaces follow the Bianchi classification, but for practical reasons we will only consider the case of Bianchi IX model geometries that describe homogeneous but generally nonisotropic metrics on $S^{3}$. The corresponding gravitational instantons of the four-dimensional Euclidean theory are special in that they admit an $\mathrm{SU}(2)$ isometry group and they provide the simplest examples in our study. In this section, we set up the notation and present some useful formulas that will enable us to formulate the problem as an autonomous system of ordinary differential equations. The Ricci and Cotton flows are studied separately here for Bianchi IX geometries. Comparison with the gravitational instantons of ordinary gravity will also be made at the appropriate places.

\subsection{Some basic facts}

We consider four-dimensional Riemannian manifolds that are foliated by homogeneous three-dimensional spaces of the form

$$
\mathrm{d} s^{2}=\mathrm{d} t^{2}+\sum_{i} \gamma_{i}(t)\left(\sigma^{i}\right)^{2}
$$

setting $N=1$ and $N_{i}=0$. The coefficients $\gamma_{i}$ are taken to depend only on $t$ and $\sigma^{i}$ are the left-invariant Maurer-Cartan one-forms of SU(2)

$$
\begin{aligned}
\sigma^{1} & =\sin \vartheta \sin \psi \mathrm{d} \varphi+\cos \psi \mathrm{d} \vartheta \\
\sigma^{2} & =\sin \vartheta \cos \psi \mathrm{d} \varphi-\sin \psi \mathrm{d} \vartheta \\
\sigma^{3} & =\cos \vartheta \mathrm{d} \varphi+\mathrm{d} \psi
\end{aligned}
$$

with Euler angles ranging as $0 \leq \vartheta \leq \pi, 0 \leq \varphi \leq 2 \pi, 0 \leq \psi \leq 4 \pi$, which satisfy

$$
\mathrm{d} \sigma^{i}+\frac{1}{2} \epsilon_{j k}^{i} \sigma^{j} \wedge \sigma^{k}=0 .
$$

The three-dimensional leaves are, in general, homogeneous but non-isotropic threespheres. The isometry group is enhanced to $\mathrm{SU}(2) \times \mathrm{U}(1)$ when any two $\gamma_{i}$ 's coincide by imposing axial symmetry. Full isotropy requires all $\gamma_{i}$ 's to be equal, in which case the symmetry of the model is promoted to $\mathrm{SU}(2) \times \mathrm{SU}(2)$. The volume of the three-sphere is

$$
V=16 \pi^{2} \sqrt{\gamma_{1} \gamma_{2} \gamma_{3}}
$$

and when all coefficients $\gamma_{i}$ are equal to $L^{2} / 4$ the volume is expressed in terms of the radius $L$ as $V=2 \pi^{2} L^{3}$.

The Ricci and Cotton tensors are diagonal for all homogeneous geometries and this ensures consistency of the reduced models. They take the following form for the Bianchi IX class,

$$
\begin{aligned}
R_{11} & =\frac{1}{2 \gamma_{2} \gamma_{3}}\left[\gamma_{1}^{2}-\left(\gamma_{2}-\gamma_{3}\right)^{2}\right] \\
C_{11} & =-\frac{\gamma_{1}}{2\left(\gamma_{1} \gamma_{2} \gamma_{3}\right)^{3 / 2}}\left[\gamma_{1}^{2}\left(2 \gamma_{1}-\gamma_{2}-\gamma_{3}\right)-\left(\gamma_{2}+\gamma_{3}\right)\left(\gamma_{2}-\gamma_{3}\right)^{2}\right]
\end{aligned}
$$


and similarly for the other two components that follow by cyclic permutation of the indices. Also, the Ricci scalar curvature is given by

$$
R=\frac{1}{2 \gamma_{1} \gamma_{2} \gamma_{3}}\left[2 \gamma_{1} \gamma_{2}+2 \gamma_{2} \gamma_{3}+2 \gamma_{3} \gamma_{1}-\gamma_{1}^{2}-\gamma_{2}^{2}-\gamma_{3}^{2}\right]
$$

whereas the trace of the Cotton tensor vanishes, as it can be readily checked.

With these explanations in mind, we arrive at the following system of ordinary differential equations for the metric coefficients $\gamma_{i}(t)$

$$
\frac{\mathrm{d} \gamma_{i}}{\mathrm{~d} t}=-\frac{\kappa^{2}}{\kappa_{W}^{2}}\left(R_{i i}-\frac{2 \lambda-1}{2(3 \lambda-1)} R \gamma_{i}+\frac{\Lambda_{W}}{1-3 \lambda} \gamma_{i}\right)+\frac{\kappa^{2}}{w_{\mathrm{CS}}} C_{i i}
$$

as the Bianchi IX mini-superspace model of the combined Ricci-Cotton flow with general couplings.

\subsection{Ricci flow}

When $w_{\text {CS }} \rightarrow \infty$ the Cotton term decouples and one arrives at a variant of the Ricci flow as the relevant equation for Hořava-Lifshitz gravity with anisotropy scaling parameter $z=2$. In this case, the system becomes

$$
\frac{\mathrm{d} \gamma_{i}}{\mathrm{~d} t}=-\frac{\kappa^{2}}{\kappa_{W}^{2}}\left(R_{i i}-\frac{2 \lambda-1}{2(3 \lambda-1)} R \gamma_{i}+\frac{\Lambda_{W}}{1-3 \lambda} \gamma_{i}\right)
$$

and its properties resemble the ordinary Ricci flow on $S^{3}$

$$
\frac{\mathrm{d} \gamma_{i}}{\mathrm{~d} t}=-R_{i i}(\gamma)
$$

Formally, one follows from the other by rescaling the metric with a function of time and changing time variable by suitable reparametrization; in such case, the components of the Ricci tensor remain invariant and they assume the same form for the rescaled components of the metric.

The Ricci flow equations (3.10) for homogeneous geometries are well studied in the literature following the original work [47]. For Bianchi IX geometries they take the form

$$
\begin{aligned}
& \frac{2}{\gamma_{1}} \frac{\mathrm{d} \gamma_{1}}{\mathrm{~d} t}=\frac{1}{\gamma_{1} \gamma_{2} \gamma_{3}}\left[\left(\gamma_{2}-\gamma_{3}\right)^{2}-\gamma_{1}^{2}\right], \\
& \frac{2}{\gamma_{2}} \frac{\mathrm{d} \gamma_{2}}{\mathrm{~d} t}=\frac{1}{\gamma_{1} \gamma_{2} \gamma_{3}}\left[\left(\gamma_{3}-\gamma_{1}\right)^{2}-\gamma_{2}^{2}\right], \\
& \frac{2}{\gamma_{3}} \frac{\mathrm{d} \gamma_{3}}{\mathrm{~d} t}=\frac{1}{\gamma_{1} \gamma_{2} \gamma_{3}}\left[\left(\gamma_{1}-\gamma_{2}\right)^{2}-\gamma_{3}^{2}\right]
\end{aligned}
$$

and coincide with the celebrated Darboux-Halphen system that was introduced by Darboux in the nineteenth century [48] and subsequently solved by Halphen [49, 50]. ${ }^{5}$ In principle, solutions of these equations can be translated into solutions of the original system (3.9).

\footnotetext{
${ }^{5}$ For the comparison one must consider the variables $\omega_{1}=\gamma_{2} \gamma_{3}, \omega_{2}=\gamma_{1} \gamma_{3}, \omega_{3}=\gamma_{1} \gamma_{2}$ and change the time coordinate to $\mathrm{d} T=\mathrm{d} t / \gamma_{1} \gamma_{2} \gamma_{3}$. Then, the equations take the equivalent form

$$
\frac{\mathrm{d} \omega_{1}}{\mathrm{~d} T}=\omega_{2} \omega_{3}-\omega_{1}\left(\omega_{2}+\omega_{3}\right)
$$

with cyclic permutations for $\omega_{2}$ and $\omega_{3}$. The identification of the Ricci flow equations for Bianchi IX geometry with the Darboux-Halphen system has escaped attention in the mathematics literature.
} 
It can be shown quite generally that for given initial data $\gamma_{i}^{(0)}$ the metric will evolve towards the configuration $\gamma_{1}=\gamma_{2}=\gamma_{3}=0$ by making $S^{3}$ rounder and rounder until the whole space collapses to a point. A particularly simple solution that exhibits this behavior is provided by

$$
\gamma_{1}(t)=\gamma_{2}(t)=\gamma_{3}(t)=\frac{1}{2}\left(t_{0}-t\right)
$$

and describes an isotropic metric on $S^{3}$ whose radius evolves from infinitely large size to zero as $t$ varies from $-\infty$ to $t_{0}$.

Actually, following the literature [47], the convergence of the flow lines is best described in terms of the normalized Ricci flow equation on $S^{3}$

$$
\frac{\mathrm{d} \gamma_{i}}{\mathrm{~d} t}=-R_{i i}+\frac{1}{3} R \gamma_{i},
$$

which follows directly from equation (3.9) in the limit $\lambda \rightarrow-\infty$, and which can be obtained from the ordinary Ricci flow by (yet another) suitable rescaling of the metric and time reparametrization. Then, the volume is preserved along the flow and the round metric (fully isotropic model with finite radius) arises as fixed point that is exponentially reached after infinitely long time, regardless of initial conditions. The normalized Ricci flow will also be in focus later in section 4 for different reasons.

The Darboux-Halphen system is not algebraically integrable when $\gamma_{1} \neq \gamma_{2} \neq \gamma_{3}$. All its solutions, however, can be expressed in terms of modular forms (see, for instance, [51]). The system becomes algebraically integrable when two $\gamma_{i}$ 's are equal. Then, the corresponding three-dimensional space is a three-sphere with axially symmetric metric. Setting $\gamma_{1}=\gamma_{2} \geq$ $\gamma_{3}$, the system (3.11) simplifies and exhibits a first integral

$$
\frac{1}{\gamma_{3}^{2}}-\frac{1}{\gamma_{1} \gamma_{3}}=\frac{1}{4 m^{2}}
$$

with arbitrary parameter $m$. The solution is subsequently described as

$$
\frac{\gamma_{1}}{m}+\operatorname{arcsinh} \frac{\gamma_{1}}{m}=\frac{-t+t_{0}}{m}=\log \frac{2 m+\gamma_{3}}{2 m-\gamma_{3}}-2 m\left(\frac{1}{2 m+\gamma_{3}}-\frac{1}{2 m-\gamma_{3}}\right),
$$

using another integration constant $t_{0}$. The fully isotropic solution with $\mathrm{SU}(2) \times \mathrm{SU}(2)$ isometry is obtained by taking the limit $m \rightarrow \infty$.

Remarkably, the same system of equations arises in the description of a class of selfdual instantons with $\mathrm{SU}(2)$ isometry in ordinary gravity [44], as well as in the description of the moduli space of $\mathrm{SU}(2)$ BPS monopoles with magnetic charge 2 [52-54]. In these two cases, which share many features with each other, the ansatz for the Bianchi IX geometries takes the form

$$
\mathrm{d} s^{2}=\mathrm{d} t^{2}+a^{2}(t)\left(\sigma^{1}\right)^{2}+b^{2}(t)\left(\sigma^{2}\right)^{2}+c^{2}(t)\left(\sigma^{3}\right)^{2}
$$

with $a(t)=\gamma_{1}(t), b(t)=\gamma_{2}(t)$ and $c(t)=\gamma_{3}(t)$ satisfying the same system of equations (3.11) above. Then, the corresponding axially symmetric gravitational instanton with $\gamma_{1}=\gamma_{2}$ is the self-dual Taub-NUT metric with NUT parameter $m$. It can be brought into 
standard form using a radial coordinate $r \geq m$ with $m \operatorname{arcosh}(r / m)+\sqrt{(r-m)(r+m)}=$ $-t+t_{0}$ so that the solution (3.15) becomes

$$
\gamma_{1}=\gamma_{2}=\sqrt{(r-m)(r+m)}, \quad \gamma_{3}=2 m \sqrt{\frac{r-m}{r+m}} .
$$

The fully anisotropic instantons of Einstein gravity with $\mathrm{SU}(2)$ isometry correspond to the so called Atiyah-Hitchin metric [52-54], which is also the metric on the moduli space of charge 2 BPS SU(2) monopoles in general position. These metrics will be discussed further in section 6 while comparing instanton solutions of general relativity with those of Hořava-Lifshitz theory.

\subsection{Cotton flow}

Next, we consider the pure Cotton flow equations ${ }^{6}$ that arise in the limit $\kappa_{W} \rightarrow \infty$. They describe solutions of $z=3$ Hořava-Lifshitz theory when the volume of space is very small, i.e., in the deep ultra-violet regime where the Cotton term dominates and all subleading relevant operators can be safely dropped from the potential. Then, the equations for Bianchi IX geometries take the form

$$
\begin{aligned}
& \frac{2 w_{\mathrm{CS}}}{\kappa^{2} \gamma_{1}} \frac{\mathrm{d} \gamma_{1}}{\mathrm{~d} t}=-\frac{1}{\left(\gamma_{1} \gamma_{2} \gamma_{3}\right)^{3 / 2}}\left[\gamma_{1}^{2}\left(2 \gamma_{1}-\gamma_{2}-\gamma_{3}\right)-\left(\gamma_{2}+\gamma_{3}\right)\left(\gamma_{2}-\gamma_{3}\right)^{2}\right], \\
& \frac{2 w_{\mathrm{CS}}}{\kappa^{2} \gamma_{2}} \frac{\mathrm{d} \gamma_{2}}{\mathrm{~d} t}=-\frac{1}{\left(\gamma_{1} \gamma_{2} \gamma_{3}\right)^{3 / 2}}\left[\gamma_{2}^{2}\left(2 \gamma_{2}-\gamma_{3}-\gamma_{1}\right)-\left(\gamma_{3}+\gamma_{1}\right)\left(\gamma_{3}-\gamma_{1}\right)^{2}\right], \\
& \frac{2 w_{\mathrm{CS}}}{\kappa^{2} \gamma_{3}} \frac{\mathrm{d} \gamma_{3}}{\mathrm{~d} t}=-\frac{1}{\left(\gamma_{1} \gamma_{2} \gamma_{3}\right)^{3 / 2}}\left[\gamma_{3}^{2}\left(2 \gamma_{3}-\gamma_{1}-\gamma_{2}\right)-\left(\gamma_{1}+\gamma_{2}\right)\left(\gamma_{1}-\gamma_{2}\right)^{2}\right],
\end{aligned}
$$

and clearly they are much more complicated than the Darboux-Halphen system. It is not known whether they are algebraically integrable when $\gamma_{1} \neq \gamma_{2} \neq \gamma_{3}$, but it can be easily shown that, under any initial data $\gamma_{i}^{(0)}$, they flow exponentially fast towards the fixed point which is the round metric on $S^{3}$ and it is conformally flat [46]. Since the Cotton tensor is odd under parity (because of the fully antisymmetric epsilon symbol appearing in its definition), there is always an ambiguity in the overall sign of the flow equations. Here, we have chosen the sign that takes any metric towards the fixed point rather than away from it when $w_{\mathrm{CS}}>0$.

The behavior of the Cotton flow is similar to the normalized Ricci flow, as they both preserve the volume of space $V=2 \pi^{2} L^{3}$, but the convergence rate is different. To compare the two it is sufficient to linearize the corresponding equations around the fixed point

$$
\gamma_{1}=\gamma_{2}=\gamma_{3}=\frac{L^{2}}{4}
$$

by considering small perturbations of the metric coefficients

$$
\gamma_{1}(t)=\frac{L^{2}}{4}(1+\delta x(t)), \quad \gamma_{2}(t)=\frac{L^{2}}{4}(1+\delta y(t))
$$

\footnotetext{
${ }^{6}$ The Cotton flow was originally introduced in the literature [46] as an alternative to the Ricci flow for studying the existence of constant curvature metrics on three-manifolds. So far it has only been applied to homogeneous geometries and its general utility for proving the Poincaré conjecture remains questionable.
} 
whereas $\gamma_{3}(t)$ changes accordingly,

$$
\gamma_{3}(t)=\frac{L^{2}}{4}(1-\delta x(t)-\delta y(t)),
$$

so that the volume of space is preserved. Then, the autonomous system of Cotton flow equations (3.18) becomes to linear order

$$
\frac{\mathrm{d}}{\mathrm{d} t}\left(\begin{array}{l}
\delta x \\
\delta y
\end{array}\right)=-\frac{12 \kappa^{2}}{w_{\mathrm{CS}} L^{3}}\left(\begin{array}{ll}
1 & 0 \\
0 & 1
\end{array}\right)\left(\begin{array}{l}
\delta x \\
\delta y
\end{array}\right)
$$

and the two eigenvalues are equal and negative for $w_{\mathrm{CS}}>0$,

$$
\zeta_{1}=\zeta_{2}=-\frac{12 \kappa^{2}}{w_{\mathrm{CS}} L^{3}}
$$

ensuring stability in all directions around the fixed point.

The perturbations diminish exponentially fast, as

$$
\delta x(t)=A \mathrm{e}^{-t / \tau_{\mathrm{C}}}, \quad \delta y(t)=B \mathrm{e}^{-t / \tau_{\mathrm{C}}},
$$

using arbitrary integration constants $A, B$ and the characteristic time scale of dissipation

$$
\tau_{\mathrm{C}}=\frac{w_{\mathrm{CS}} L^{3}}{12 \kappa^{2}} .
$$

Thus, when $w_{\mathrm{CS}}$ is very small compared to $\kappa_{\mathrm{W}}^{2}$ so that the Cotton tensor dominates the flow over the Ricci curvature and the cosmological constant terms, or equivalently when $L$ is very small so that the volume of space is tiny, $\tau_{\mathrm{C}}$ is very small and the metric perturbations dissipate very fast at late times. ${ }^{7}$

There is an additional fixed point, which is unique, up to permutation of the axes, that arises when $\gamma_{1}=\gamma_{2}=\infty$ and $\gamma_{3}=0$ (correlated limit with $V$ held fixed). It corresponds to a squashed $S^{3}$ that is completely flattened and has zero Cotton tensor, as it can be explicitly checked. Although this configuration is degenerate in one principal direction, it has no curvature singularities and it is legitimate to consider.

Axisymmetric solutions of the Cotton flow can be constructed in closed form, as for the Ricci flow. Assuming

$$
\gamma_{1}=\gamma_{2} \equiv x \frac{L^{2}}{4}, \quad \gamma_{3}=\frac{L^{2}}{4 x^{2}}
$$

so that the volume of space is held fixed to $V=2 \pi^{2} L^{3}$, the Cotton flow equations (3.18) reduce to a single equation

$$
\frac{\mathrm{d} x}{\mathrm{~d} t}=\frac{4 \kappa^{2}}{w_{\mathrm{CS}} L^{3}} \frac{1-x^{3}}{x^{5}}
$$

\footnotetext{
${ }^{7}$ We note here for completeness, and it will be used in the next section, that the normalized Ricci flow $\mathrm{d} \gamma_{i} / \mathrm{d} t=-R_{i i}+R \gamma_{i} / 3$ can be similarly analyzed by considering small perturbations around the fully isotropic fixed point. The two eigenvalues also turn out to be equal and negative, but the corresponding characteristic time scale for dissipation depends quadratically on $L$ as $\tau_{\mathrm{R}}=L^{2} / 4$ with respect to the appropriate time coordinate. Comparison with the dissipation rate of the Cotton flow will become relevant in section 4 .
} 
which is solved as follows,

$$
\frac{-t+t_{\star}}{\tau_{\mathrm{C}}}=x^{3}-1+\log \left|x^{3}-1\right|
$$

with integration constant $t_{\star}$.

This solution has two branches. For $x \geq 1, x(t)$ changes from $+\infty$ to 1 as $t$ varies from $-\infty$ to $+\infty$; the three-sphere deforms starting from the singular configuration $\gamma_{1}=\gamma_{2}=\infty$, $\gamma_{3}=0$ and gradually becomes rounder until it reaches the isotropic fixed point after infinitely long time. For $x \leq 1, x(t)$ changes from 0 to 1 as $t$ varies from $t_{0}=t_{\star}+\tau_{\mathrm{C}}$ to $+\infty$; in this case, the three-sphere evolves from the singular configuration $\gamma_{1}=\gamma_{2}=0$, $\gamma_{3}=\infty$ towards the isotropic fixed point.

Finally, we point out that there is no known solution for the fully anisotropic model geometry that is analogous to the general solution of the Darboux-Halphen system. It remains an open question whether the system is algebraically integrable and find its solution.

\section{Normalized Ricci-Cotton flow}

We will now investigate the combined Ricci-Cotton flow when the effective speed of light vanishes by letting $\lambda \rightarrow-\infty$. In this case, the flow equations take the following general form,

$$
\partial_{t} g_{i j}=-\frac{\kappa^{2}}{\kappa_{W}^{2}}\left(R_{i j}-\frac{1}{3} R g_{i j}\right)+\frac{\kappa^{2}}{w_{\mathrm{CS}}} C_{i j}
$$

and they become independent of $\Lambda_{W}$. The driving curvature term is traceless and the deformations preserve the volume of space. Thus, the resulting normalized Ricci-Cotton flow is a superposition of the Cotton and the normalized Ricci flow with competing effects, in general, that depend on the sign of $w_{\mathrm{CS}}$.

\subsection{The general system of Bianchi IX equations}

Using the Bianchi IX ansatz for the three-dimensional geometry, the normalized RicciCotton flow equations form an autonomous system of equations for the coefficients $\gamma_{1}, \gamma_{2}$, $\gamma_{3}$. Since the volume $V=2 \pi^{2} L^{3}$ is conserved, it is convenient to use two independent variables $x(t)$ and $y(t)$,

$$
\gamma_{1}=\frac{x L^{2}}{4}, \quad \gamma_{2}=\frac{y L^{2}}{4}, \quad \gamma_{3}=\frac{L^{2}}{4 x y}
$$

and also set

$$
\tau=\frac{4 \kappa^{2}}{\kappa_{W}^{2} L^{2}} t, \quad \mu=\frac{w_{\mathrm{CS}} L}{\kappa_{W}^{2}} .
$$

Then, the general system of Bianchi IX equations takes the form

$$
\begin{aligned}
& \frac{\mathrm{d} x}{\mathrm{~d} \tau}=\frac{1}{\mu x^{2} y^{3}}\left\{\left(1+\frac{\mu}{3} x y+x y^{2}\right)\left(1-x y^{2}\right)^{2}+\left(\frac{\mu}{3}+x\right)\left(1+x y^{2}-2 x^{2} y\right) x^{3} y^{2}\right\} \\
& \frac{\mathrm{d} y}{\mathrm{~d} \tau}=\frac{1}{\mu x^{3} y^{2}}\left\{\left(1+\frac{\mu}{3} x y+x^{2} y\right)\left(1-x^{2} y\right)^{2}+\left(\frac{\mu}{3}+y\right)\left(1+x^{2} y-2 x y^{2}\right) x^{2} y^{3}\right\} .
\end{aligned}
$$




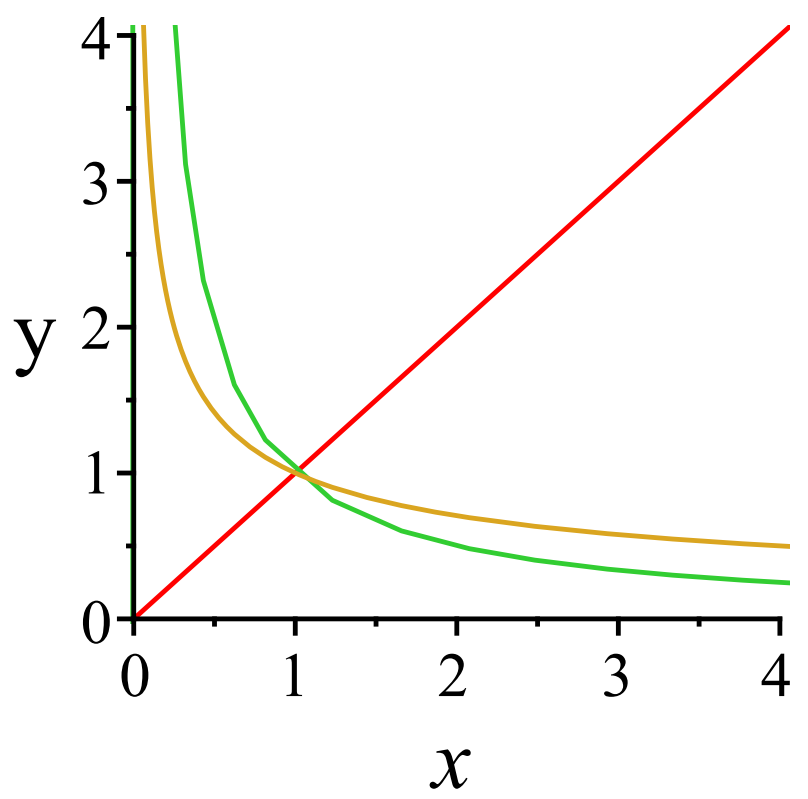

Figure 1. The lines of enhanced $\mathrm{SU}(2) \times \mathrm{U}(1)$ symmetry in the $(x, y)$ plane.

It is not known whether this system of equations is integrable, in general. However, we will be able to characterize its fixed points and study their stability on general grounds in order to infer the qualitative behavior of the flow lines. Note that the individual components of our system, the normalized Ricci flow and the Cotton flow, can compete with each other. When $\mu>0\left(w_{\mathrm{CS}}>0\right)$, they both work in the same direction, but when $\mu<0$ $\left(w_{\mathrm{CS}}<0\right)$ they work against each other and can affect the form and stability properties of the fixed points.

It will also be seen later that these equations can be solved exactly in the axially symmetric case $x=y$. Actually, there are three curves of axial symmetry in the problem, but, in practice, it is sufficient to consider only one of these axially symmetric cases, since the other two follow by permutation of the principal axes of $S^{3}$. Thus, apart from $x=y$, we also have $x^{2} y=1$ and $x y^{2}=1$, depending on the pair of $\gamma_{i}$ 's that become equal and reduce the flow equations to a single one. They correspond to metrics on $S^{3}$ with enhanced symmetry $\mathrm{SU}(2) \times \mathrm{U}(1)$ and they all intersect at the fully isotropic point $x=y=1$. These curves are by themselves flow lines, which, however, cannot be crossed by other flow lines; if any two $\gamma_{i}$ 's become equal at a given (finite) time they will remain equal for ever. Therefore, these three curves provide the barriers for six regions in the $(x, y)$ plane where the generic flow lines are confined depending on initial conditions. The maximal time range of any given flow line also depends on the region in which the flow is confined. Finally, the flow lines along the three curves of axial symmetry can reach the fully isotropic point but cannot continue running beyond it. The three curves of axial symmetry are depicted in figure 1 , which is restricted to the first quadrant of the $(x, y)$ plane so that the metric has physical signature, and they intersect at $(1,1)$. 
These general qualitative remarks are sufficient to illustrate the evolution of any given initial data on the $(x, y)$ plane, provided that all fixed points are found and the arrows of flow lines around them are correctly identified by stability analysis. The same remarks apply equally well to the normalized Ricci and Cotton flows which can arise separately as special cases.

\subsection{Classification of the fixed points}

We are going now to classify all fixed points of the system (4.4) and (4.5) and find the critical values of $\mu$ that separate their behavior into different phases of stability.

The isotropic fixed point. The metric on the round sphere is a fixed point of the normalized Ricci-Cotton flow for all values of $\mu$ (positive and negative) and corresponds to the point

$$
x_{\star}=y_{\star}=1
$$

on the $(x, y)$ plane. Its stability, however, depends on the values of the parameter $\mu$.

By considering small perturbations around this fixed point, as

$$
x(t)=x_{\star}+\delta x(t), \quad y(t)=y_{\star}+\delta y(t),
$$

we find that the linearized system of equations takes the form

$$
\frac{\mathrm{d}}{\mathrm{d} \tau}\left(\begin{array}{l}
\delta x \\
\delta y
\end{array}\right)=-\left(1+\frac{3}{\mu}\right)\left(\begin{array}{ll}
1 & 0 \\
0 & 1
\end{array}\right)\left(\begin{array}{l}
\delta x \\
\delta y
\end{array}\right)
$$

The two eigenvalues are equal

$$
\zeta_{1}=\zeta_{2}=-\left(1+\frac{3}{\mu}\right)
$$

and follow by linear superposition of the corresponding eigenvalues of the normalized Ricci and Cotton flows discussed in the previous section.

The fixed point is absolutely stable when $\mu$ satisfies the bound $3 / \mu>-1$, i.e., $\mu>0$ or $\mu<-3$. In these cases, the flow line converge towards the fixed point from all directions. Otherwise, for $-3<\mu<0$, the isotropic point is absolutely unstable and the flow lines diverge away from it in all directions. Finally, when $\mu=-3$, the eigenvalues are zero and all points in the vicinity of the fixed point are at equilibrium. Thus,

- $\mu>0$ or $\mu<-3$ : absolutely stable fixed point,

- $-3<\mu<0$ : absolutely unstable fixed point.

These results are in exact agreement with the competition between the Ricci and Cotton components of the flow and can be understood by comparing the characteristic 
time scales $\tau_{\mathrm{R}}$ and $\tau_{\mathrm{C}}$ that govern metric perturbations at late times. ${ }^{8}$ For $\mu>0$ both components dissipate all metric perturbations exponentially fast, but for $\mu<0$ the Cotton flow contributes differently leading to exponential growth of the perturbations. For the critical value $\mu=-3$, the dissipation of the normalized Ricci flow is canceled by the exponential growth of the Cotton flow, making zero the characteristic matrix of metric perturbations.

Anisotropic fixed points. For negative $\mu$ there are additional fixed points that correspond to particular axisymmetric metrics. As such, they appear in three copies related by permutation of the axes of $S^{3}$ and they are located on the lines of axial symmetry in the $(x, y)$ plane. In particular, they arise

- on the diagonal $x=y$ with $x_{\star}=\sqrt{-3 / \mu}$,

- on the branch $x^{2} y=1$ with $x_{\star}=\sqrt{-3 / \mu}$,

- on the branch $x y^{2}=1$ with $y_{\star}=\sqrt{-3 / \mu}$.

Note that all these points coalesce with the fully isotropic point when $\mu$ assumes the critical value $\mu=-3$.

It suffices to perform stability analysis around one of these fixed points, say the one located on the diagonal line, since the results will be identical for all of them by the symmetry of the problem. Using small fluctuations around the fixed point

$$
x_{\star}=y_{\star}=\sqrt{-\frac{3}{\mu}}
$$

so that $x(t)=x_{\star}+\delta x(t)$ and $y(t)=y_{\star}+\delta y(t)$, the linearized system takes the form,

$$
\frac{\mathrm{d}}{\mathrm{d} \tau}\left(\begin{array}{c}
\delta x \\
\delta y
\end{array}\right)=\frac{1}{2}\left(\begin{array}{l}
\zeta_{1}+\zeta_{2} \zeta_{1}-\zeta_{2} \\
\zeta_{1}-\zeta_{2} \zeta_{1}+\zeta_{2}
\end{array}\right)\left(\begin{array}{c}
\delta x \\
\delta y
\end{array}\right),
$$

where

$$
\begin{aligned}
\zeta_{1} & =\frac{2}{3} \sqrt{-\frac{\mu}{3}}\left[\left(-\frac{\mu}{3}\right)^{3 / 2}-1\right] \\
\zeta_{2} & =\frac{2}{3} \sqrt{-\frac{\mu}{3}}\left[\left(-\frac{3}{\mu}\right)^{3 / 2}-1\right]\left[4\left(-\frac{3}{\mu}\right)^{3 / 2}-1\right] .
\end{aligned}
$$

${ }^{8}$ According to definitions we have the following relations

$$
\mu=\frac{w_{\mathrm{CS}} L}{\kappa_{W}^{2}}=3 \frac{\tau_{\mathrm{C}}}{\tau_{\mathrm{R}}} \quad \text { with } \quad \tau_{\mathrm{C}}=\frac{w_{\mathrm{CS}} L^{3}}{12 \kappa^{2}}, \quad \tau_{\mathrm{R}}=\frac{\kappa_{W}^{2} L^{2}}{4 \kappa^{2}} .
$$

The characteristic time scales $\tau_{\mathrm{R}}$ and $\tau_{\mathrm{C}}$ refer to the original time coordinate $t$ but their ratio is the same in the time coordinate $\tau$. 
The two eigenvalues are $\zeta_{1}$ and $\zeta_{2}$ and they are unequal offering various possibilities in general. They both vanish for $\mu=-3$. Otherwise, we have the following cases depending on the sign of the eigenvalues:

- $-3<\mu<0$ : saddle fixed point with $\zeta_{1}<0<\zeta_{2}$,

- $-6 \sqrt[3]{2}<\mu<-3: \quad$ saddle fixed point with $\zeta_{2}<0<\zeta_{1}$,

- $\mu<-6 \sqrt[3]{2}: \quad$ absolutely unstable fixed point with $\zeta_{i}>0$.

Thus, the axisymmetric fixed points are never absolutely stable. They are saddle or unstable depending on $\mu$.

Totally anisotropic fixed points. It is not obvious from the beginning whether there are any fixed points with $\gamma_{1} \neq \gamma_{2} \neq \gamma_{3}$. Close inspection of the equations, assisted by numerical scanning, reveals the presence of two totally anisotropic fixed points that coexist with the axially symmetric anisotropic fixed point ${ }^{9}$ when $\mu<-6 \sqrt[3]{2}$. In fact, by the $\mathbb{Z}_{3}$ symmetry of the problem, there are six such additional fixed points, but we only focus attention on two of them appearing symmetrically left and right of the diagonal line $x=y$ in the lower two (out of the six disconnected) regions shown in figure1; their presence should not be confused with the mirror images of the axially symmetric anisotropic fixed point discussed earlier.

The characteristic property of the totally anisotropic fixed points is that their Ricci scalar curvature vanishes and their location on the $(x, y)$ plane is given by

$$
x+\frac{1}{\sqrt{x}}=-\frac{\mu}{4}=y+\frac{1}{\sqrt{y}} \quad \text { with } \quad x \neq y .
$$

Of course, one can always find Bianchi IX metrics with zero scalar curvature by imposing the appropriate algebraic condition on the metric coefficients, but these are not fixed points of the flow lines for general values of $\mu$. Remarkably, they are real solutions of $\mathrm{d} x / \mathrm{d} \tau=$ $0=\mathrm{d} y / \mathrm{d} \tau$ with $x \neq y$, which coexist with the axially symmetric anisotropic fixed point when $\mu<-6 \sqrt[3]{2}$. They are not present when $\mu>-6 \sqrt[3]{2}$, since there are no real solutions to equation (4.14) in that case. We also note that when $\mu=-6 \sqrt[3]{2}$ the totally anisotropic fixed points coalesce with the axially symmetric anisotropic fixed point. Furthermore, there are no other fixed points in the problem.

The location of these fixed points is depicted in figure 2. Here, we plot the ratio $x / y$ of the anisotropic fixed points as function of $\mu$ (actually $-\mu$ ). The horizontal line represents the axially symmetric anisotropic fixed point that exists below and above the critical value of $\mu$.

At this point, it is instructive to consider the Ricci scalar curvature of the axially symmetric anisotropic fixed point $x_{\star}=y_{\star}=\sqrt{-3 / \mu}$, which turns out to be

$$
R=-\frac{2 \mu}{9 L^{2}}\left(\mu+12 \sqrt{-\frac{3}{\mu}}\right) .
$$

\footnotetext{
${ }^{9}$ We thank Christos Sourdis for pointing out the presence of these additional fixed points and thoroughly investigating their properties. This analysis was missed in a previous version of our paper and we are indebted to him for providing all the details. Similar results also appeared in [55].
} 


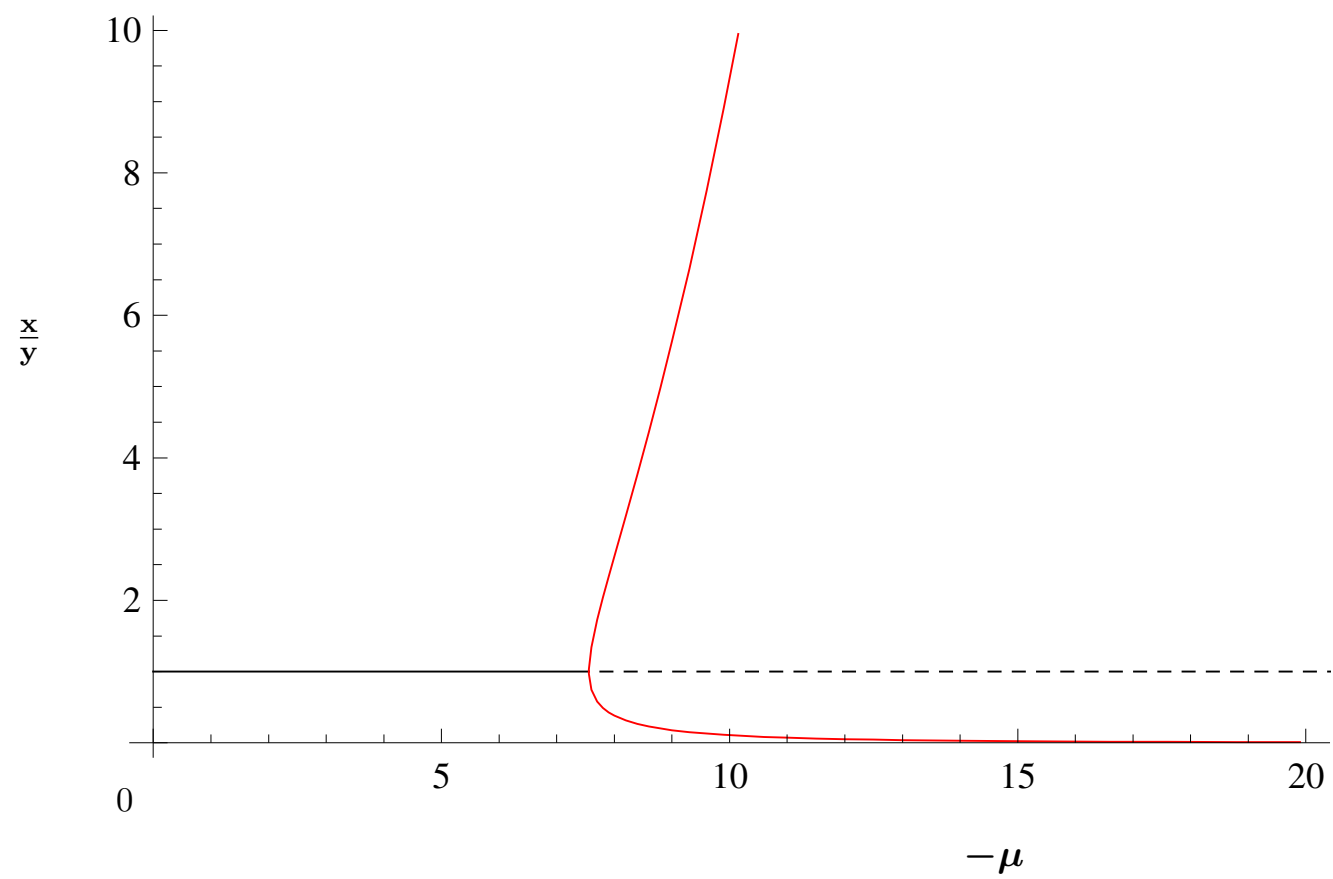

Figure 2. Relative location of all anisotropic fixed points for $\mu<0$.

When $\mu=0$ this point is at infinity and the curvature is zero. As $\mu$ varies from 0 to -3 the axially symmetric anisotropic fixed point is approaching the isotropic fixed point and the curvature increases monotonically. The curvature becomes maximal when these two points coincide at the critical value $\mu=-3$, and, then it decreases monotonically as $\mu$ varies from -3 to $-6 \sqrt[3]{2}$. It becomes zero at the other critical value $\mu=-6 \sqrt[3]{2}$, and, then, it turns negative for $\mu<-6 \sqrt[3]{2}$. The value $\mu=-6 \sqrt[3]{2}$ is also critical for the creation of the totally anisotropic fixed points, which pop out symmetrically from the diagonal and have zero curvature for all lower values of the parameter $\mu$.

These additional fixed points appear to be saddle points, as can be verified by numerical investigation for different values of $\mu$. It is not easy to obtain closed formulas for the eigenvalues of the characteristic matrix describing small perturbations around them. However, their stability properties are important for constructing instanton solutions of Hořava-Lifshitz gravity, as will be seen in detail later in section 6. Another characteristic property of these points that will also be used later is the universal value of the gravitational Chern-Simons functional. Explicit calculation shows that $W_{\mathrm{CS}}$ for the fully anisotropic fixed points is

$$
W_{\mathrm{CS}}=\frac{80 \pi^{2}}{w_{\mathrm{CS}}},
$$

which is independent of $\mu$ ! It actually coincides with the value of $W_{\mathrm{CS}}$ for the axially symmetric anisotropic fixed point when $\mu=-6 \sqrt[3]{2}$ and it is ten times larger than the value of $W_{\mathrm{CS}}$ evaluated at the totally isotropic fixed point.

An important remark is in order at this point. The equations that determine the fixed points of the normalized Ricci-Cotton flow provide only the traceless part of the classical 
equations of motion of topologically massive gravity, leaving the trace undetermined. Then, depending on the value of their Ricci scalar curvature, these points also satisfy the trace equation $R=6 \Lambda_{\mathrm{W}}$ for appropriately chosen effective value of $\Lambda_{\mathrm{W}}$. Fixed points with positive, negative or zero Ricci scalar curvature are vacua of topologically massive gravity with positive, negative or zero cosmological constant, respectively. As a result, the fixed points of the Ricci-Cotton flow with general couplings, which are the vacua of topologically massive gravity, are expected to be less than the fixed points of the normalized flow, and, in fact, they can be obtained from them in certain ways. ${ }^{10}$ This, however, does not make our analysis in section 5 redundant since their location, volume and stability properties also depend crucially on the flow equations we are considering in each case.

Summarizing the results for the normalized Ricci-Cotton flow, we note that the fully isotropic metric on $S^{3}$ is the unique fixed point for $\mu>0$ that attracts all trajectories starting from any point in the first quadrant of the $(x, y)$ plane. For $\mu<0$, there are various possibilities that result to attractive or repelling directions around the fixed points. Note that the isotropic point becomes absolutely unstable when $-3<\mu<0$, in which case the anisotropic fixed point is a saddle that attracts partially the flow lines. For $-6 \sqrt[3]{2} \leq \mu<0$ there are four in total fixed points, including their $\mathbb{Z}_{3}$ mirrors, whereas for $\mu<-6 \sqrt[3]{2}$ the total number of fixed points is ten.

\subsection{Phase portraits of the flow}

The qualitative behavior of the flow lines is illustrated by the phase portraits shown below for all possible values of $\mu$.

For positive $\mu$, which is qualitatively the same as for the normalized Ricci flow and the Cotton flow, the phase portrait is given in figure 3 .

For negative $\mu$ the stability properties of the fixed points is different in the intervals $-3<\mu<0,-6 \sqrt[3]{2}<\mu<-3$ and $\mu<-6 \sqrt[3]{2}$ and all these possibilities are represented in figures 4,5 and 6 , respectively. For later reference, it is important to realize the existence of trajectories interpolating between different fixed points. In figure 4 there is only one such flow line since one fixed point is unstable and the other a saddle. The same applies to figure 5 where the interpolating flow line connects a stable fixed point with a saddle. The picture changes drastically in figure 6 since there are infinitely many flow lines interpolating between an unstable and a stable fixed point. Also, in this case, there are additional fixed points away from the diagonal, which are saddle points. Figure 6 contains two such totally anisotropic fixed points located at $x \simeq 0.19, y \simeq 1.75$ (and $x \simeq 1.75, y \simeq 0.19$ ) for $\mu=-10<-6 \sqrt[3]{2} \simeq-7.56$. There are flow lines connecting these fixed points with the other two lying on the diagonal, but they are not easily seen on the phase portrait due to numerical deficiency.

\footnotetext{
${ }^{10}$ The mathematical problem one has to solve to determine the set of fixed points of massive gravity for given $\Lambda_{\mathrm{W}}$ is to fix $R$ instead of the volume of the normalized fixed points, as functions of $\mu$ and $L$, and deduce from it the allowed range of $\mu$ for the selected set of fewer points.
} 


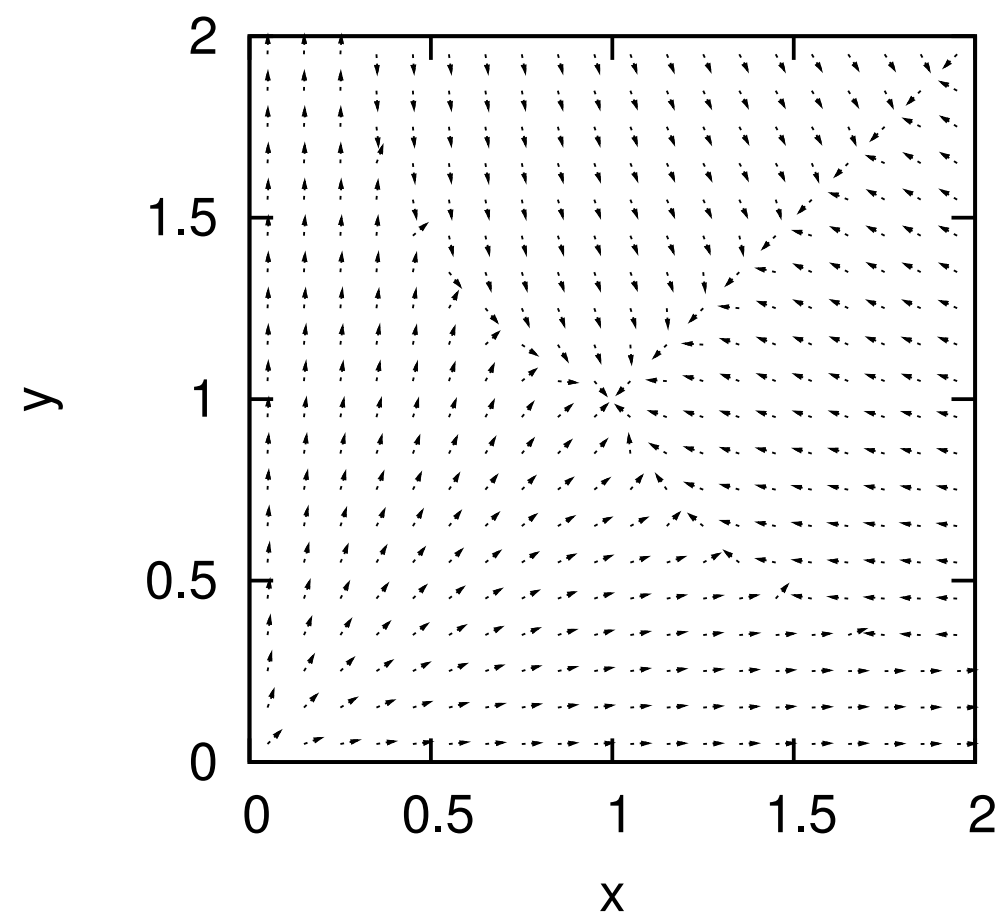

Figure 3. The flow lines for $\mu>0$; here, $\mu=2$.

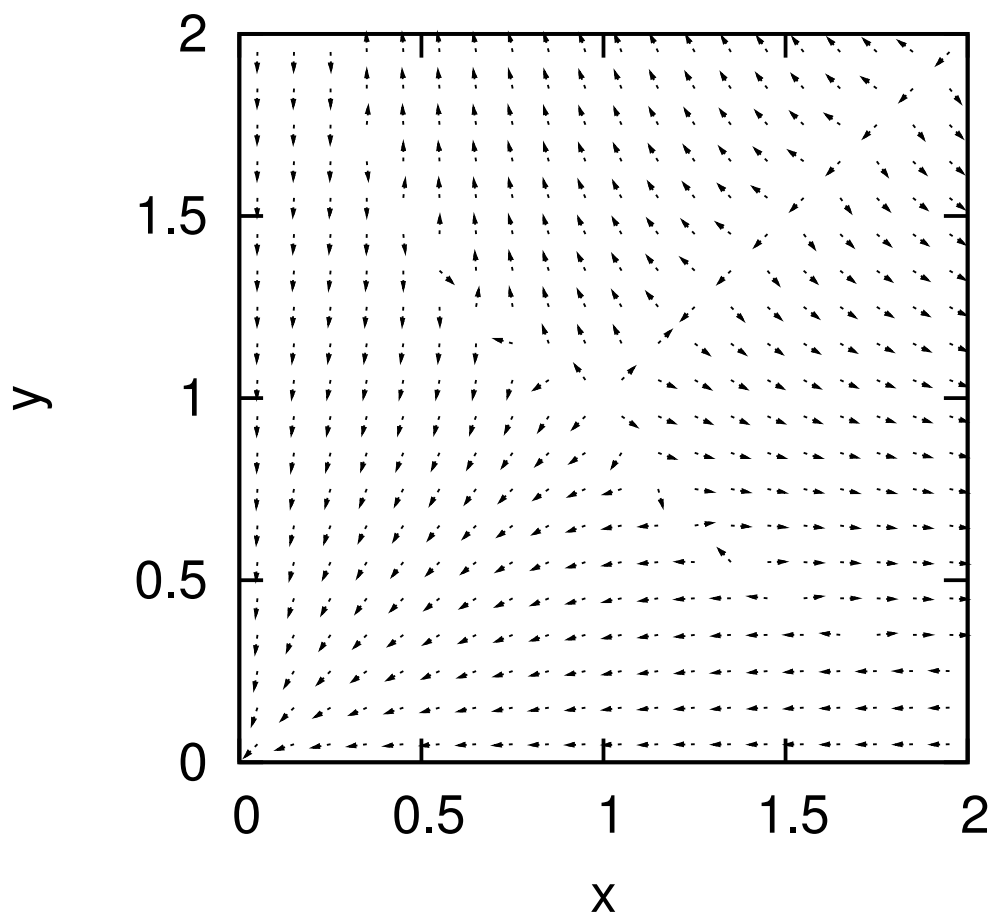

Figure 4. The flow lines for $-3<\mu<0$; here, $\mu=-2$. 


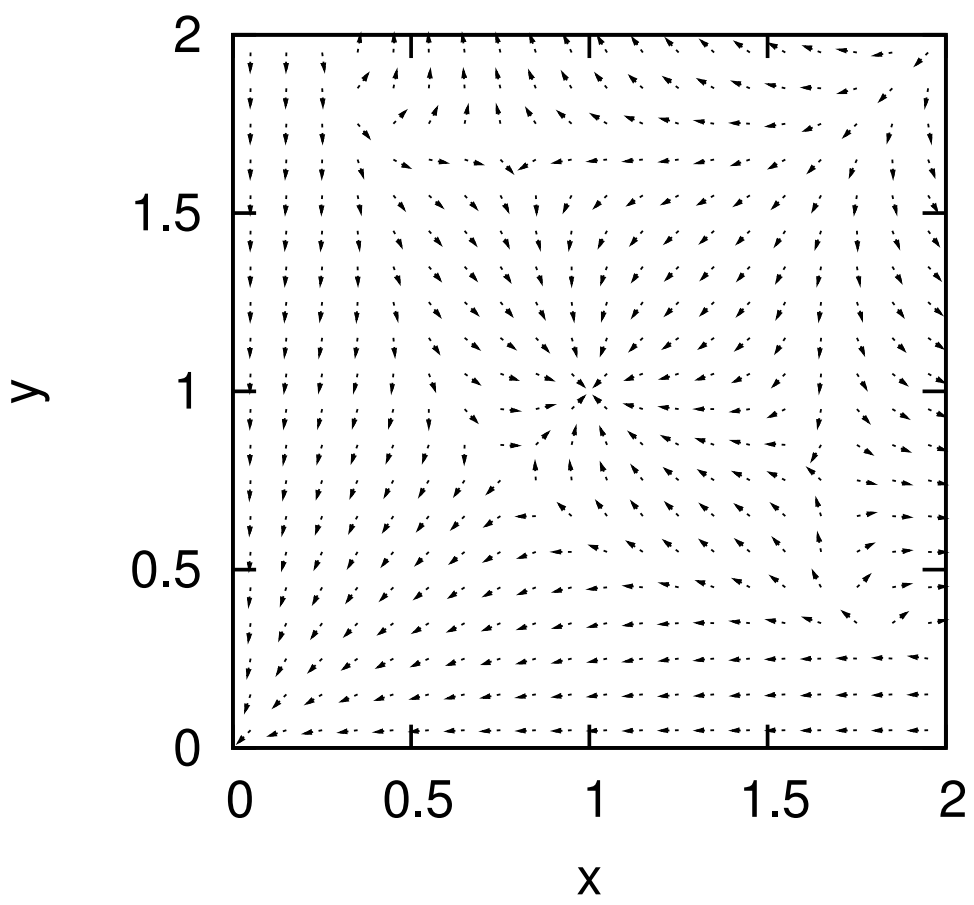

Figure 5. The flow lines for $-6 \sqrt[3]{2}<\mu<-3$; here, $\mu=-5$.

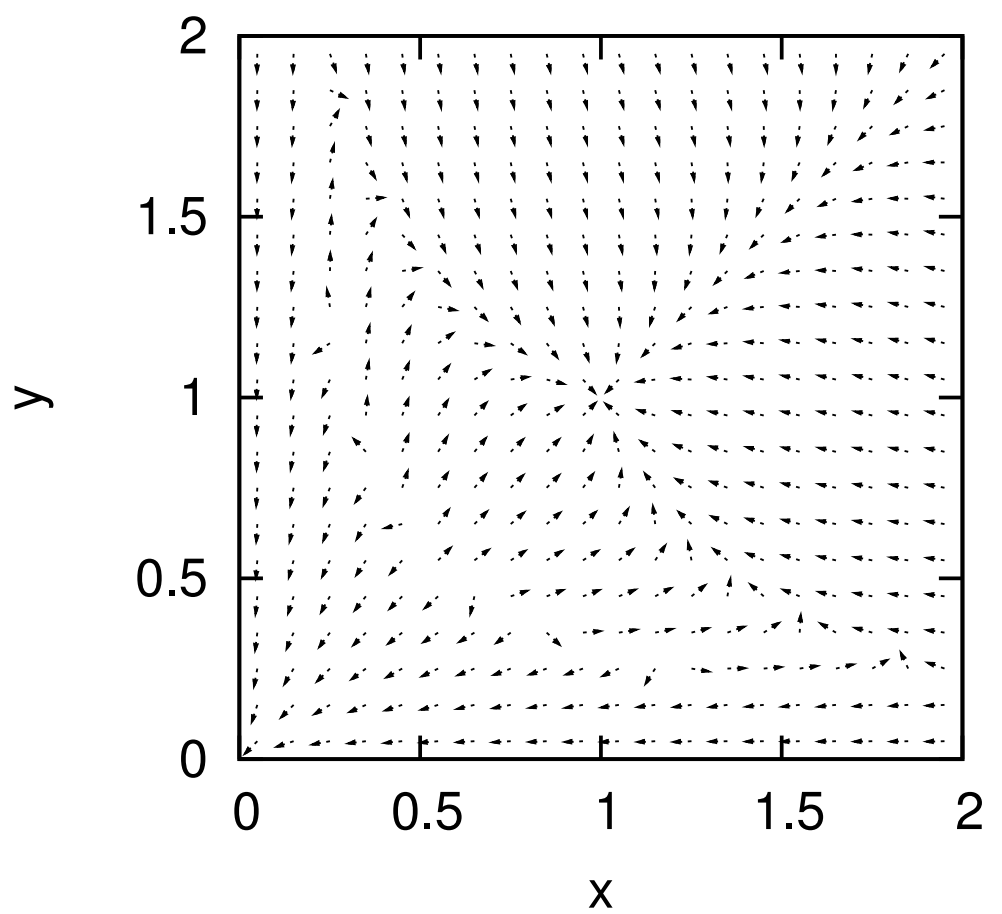

Figure 6. The flow lines for $\mu<-6 \sqrt[3]{2}$; here, $\mu=-10$. 


\subsection{Axisymmetric solutions}

Here, we present the exact axially symmetric solution of the normalized Ricci-Cotton flow, setting $x=y$. As such, it generalizes the axially symmetric solutions of the normalized Ricci and the Cotton flows discussed in the previous section. The system (4.4) and (4.5) reduces to a single equation

$$
\frac{\mathrm{d} x}{\mathrm{~d} \tau}=\frac{1}{\mu}\left(\frac{1}{x^{3}}-1\right)\left(\frac{1}{x^{2}}+\frac{\mu}{3}\right),
$$

and the flow takes place on the diagonal line of the $(x, y)$ plane. The flow connects either the origin or infinity with a fixed point or it can extend between two different fixed points. These regions do not communicate with each other and the time interval that supports the solutions depends on $\mu$ and the choice of trajectory.

The behavior around $x=0$ and $x \rightarrow \infty$ is universal and can be extracted directly from equation (4.17),

- $x \approx\left(\frac{6}{\mu}\left(\tau-\tau_{0}\right)\right)^{1 / 6}, \quad$ as $x \rightarrow 0$,

- $x \approx-\frac{1}{3} \tau, \quad$ as $x \rightarrow \infty$.

Around the fixed points the time dependence is exponential and determined by the eigenvalues $\zeta$ as $x-x_{\star} \approx \exp (\zeta \tau)$. The relevant eigenvalue for the isotropic point is given by (4.9) and for the anisotropic by (4.12).

Case I: $\boldsymbol{\mu}>\boldsymbol{0}$. The solution behaves similarly for all positive values of $\mu$, but it looks different on the two sides of the isotropic fixed point $x_{\star}=1$. We find that

$\underline{x>1}: \quad-\infty<\tau<+\infty$ as $x$ decreases from $+\infty$ to 1,

$\underline{x<1}: \quad \tau_{0}<\tau<+\infty$ as $x$ increases from $x\left(\tau_{0}\right)=0$ to 1.

The exact solution reads

$$
\begin{aligned}
\tau-\tau_{\star}= & -3 x+\frac{\mu(\mu-6)}{2\left(\mu^{2}-3 \mu+9\right)} \log \left(x^{2}+x+1\right)+\frac{\sqrt{3} \mu^{2}}{\mu^{2}-3 \mu+9} \arctan \frac{2 x+1}{\sqrt{3}} \\
& -\frac{\mu}{\mu+3} \log |x-1|+\frac{27 \mu}{2\left(\mu^{3}+27\right)} \log \left(\mu x^{2}+3\right) \\
& +\frac{81}{\mu^{3}+27} \sqrt{\frac{3}{\mu}} \arctan \left(x \sqrt{\frac{\mu}{3}}\right)
\end{aligned}
$$

and it is represented by figure 7 with appropriately chosen integration constant $\tau_{\star}$.

Case II: $\boldsymbol{\mu}<0$. In this case the solution depends on the particular value of $\mu$. First, we present the result for the critical value $\mu=-3$, which is simpler,

$$
\begin{aligned}
\tau-\tau_{\star}= & -3 x+\frac{1}{2(x-1)}+\frac{1}{\sqrt{3}} \arctan \frac{2 x+1}{\sqrt{3}}-\frac{7}{4} \log |x-1| \\
& +\frac{3}{4} \log (x+1)+\frac{1}{2} \log \left(x^{2}+x+1\right) .
\end{aligned}
$$




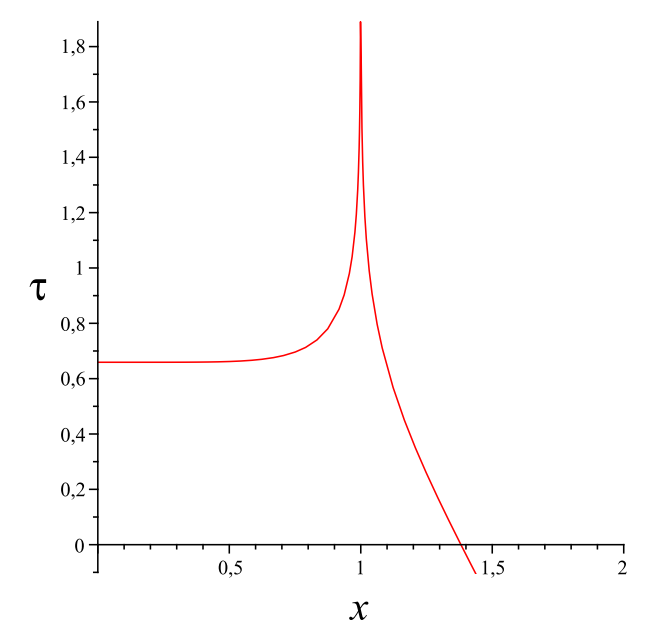

Figure 7. The dependence $\tau(x)$ for positive $\mu$; here, $\mu=1$.

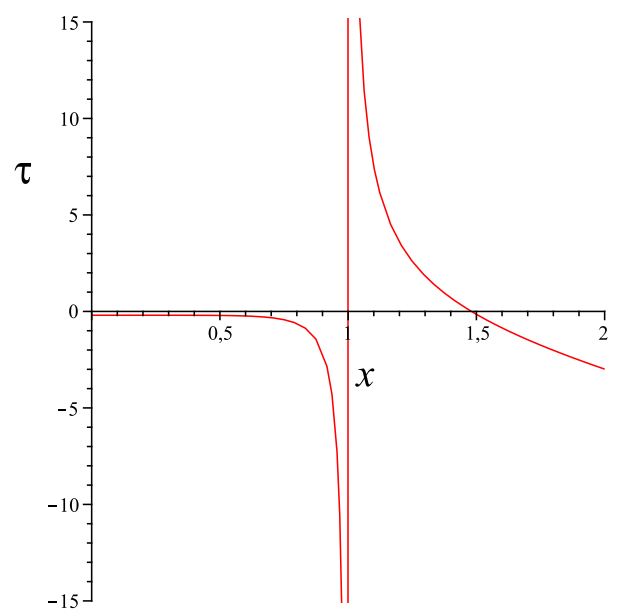

Figure 8. The dependence $\tau(x)$ for the critical value critical $\mu=-3$.

All fixed points coalesce to the isotropic and the eigenvalues vanish, so that there are no arrows infinitesimally close to this point. However, this behavior is lifted at second order in perturbation theory and there are arrows pointing from large to small values of $\tau$. This particular case is depicted in figure 8, which also shows the range of $\tau$ in the two branches.

For $\mu \neq-3$ the expression becomes much more involved and reads

$$
\begin{aligned}
\tau-\tau_{\star}= & -3 x+\frac{\mu(\mu-6)}{2\left(\mu^{2}-3 \mu+9\right)} \log \left(x^{2}+x+1\right)+\frac{\sqrt{3} \mu^{2}}{\mu^{2}-3 \mu+9} \arctan \frac{2 x+1}{\sqrt{3}} \\
& -\frac{\mu}{\mu+3} \log |x-1|+\frac{27 \mu}{2\left(\mu^{3}+27\right)} \log \left|\mu x^{2}+3\right| \\
& -\frac{81}{2\left(\mu^{3}+27\right)} \sqrt{-\frac{3}{\mu}}\left[\log \left|1-x \sqrt{-\frac{\mu}{3}}\right|-\log \left|1+x \sqrt{-\frac{\mu}{3}}\right|\right] .
\end{aligned}
$$

Then, depending on whether $-3<\mu<0$ or $\mu<-3$, the function $\tau(x)$ looks different. 


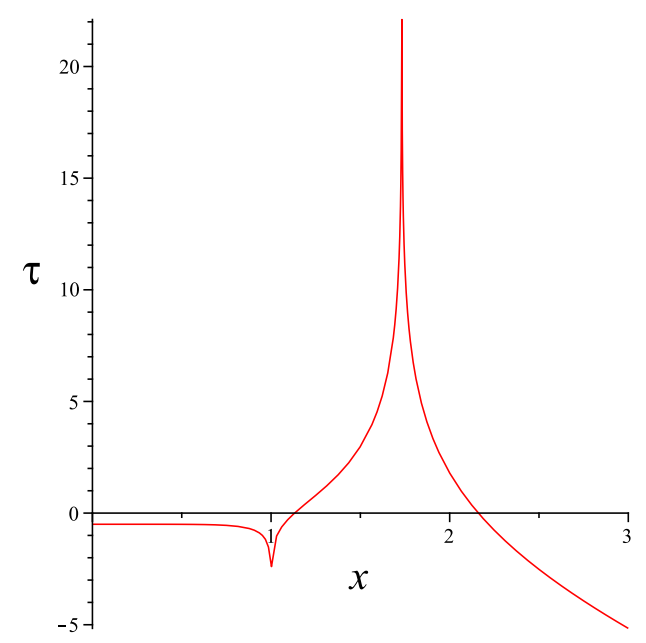

Figure 9. The dependence $\tau(x)$ for $-3<\mu<0$; here, $\mu=-1$.

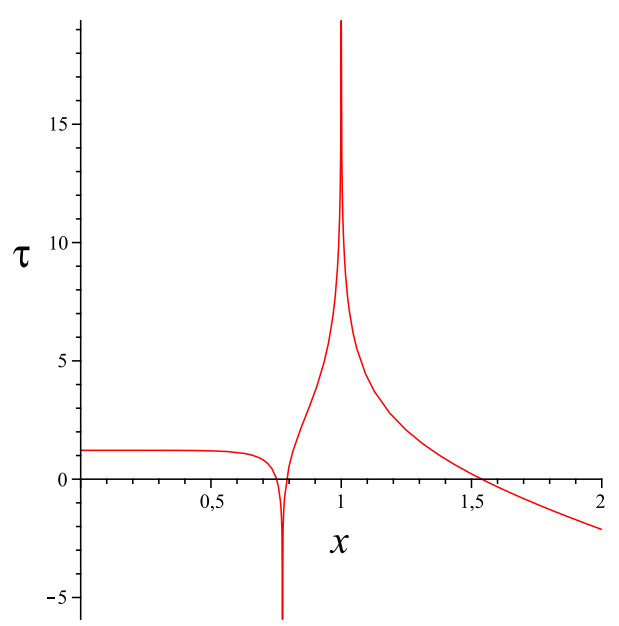

Figure 10. The dependence $\tau(x)$ for $\mu<-3$; here, $\mu=-5$.

In all these cases the solution has three branches but the range of time is not the same. We have, in particular, the following behavior depending on $\mu$ :

- For $-3<\mu<0$ the two fixed points are ordered as $x_{\star}^{\text {iso }}=1<x_{\star}^{\text {aniso }}=\sqrt{-3 / \mu}$ with the isotropic being repulsive and the anisotropic attractive. The exact solution is represented by figure 9 .

- For $\mu<-3$ the two fixed points are ordered differently, as $x_{\star}^{\text {aniso }}=\sqrt{-3 / \mu}<x_{\star}^{\text {iso }}=1$. The isotropic point is now attractive whereas the anisotropic is repulsive. Then, the exact solution along the diagonal is represented by figure 10 and there is no essential difference for $\mu$ below or above the value $-6 \sqrt[3]{2}$.

Note that the three branches shown in figures 9 and 10 range differently: the first has support on a semi-infinite time interval, whereas the second and third to the right 
are eternal solutions that exist for all time $-\infty<\tau<+\infty$. (Despite appearances, caused by numerical deficiency, the spike at $x=1$ extends to infinity in figure 9 ).

\section{Ricci-Cotton flow with general couplings}

Let us now examine the Ricci-Cotton flow equations for Bianchi IX model geometries with arbitrary couplings by letting $\lambda$ and $\Lambda_{W}$ take arbitrary values. The system of equations that needs to be studied is provided by $(3.8)$

$$
\frac{\mathrm{d} \gamma_{i}}{\mathrm{~d} \tau}=-R_{i i}+\frac{2 \lambda-1}{2(3 \lambda-1)} R \gamma_{i}+\frac{\Lambda_{W}}{3 \lambda-1} \gamma_{i}+\frac{1}{\mu} C_{i i}
$$

with

$$
\tau=\frac{\kappa^{2}}{\kappa_{W}^{2}} t, \quad \mu=\frac{w_{\mathrm{CS}}}{\kappa_{W}^{2}} .
$$

The definition of $\tau$ and $\mu$ resembles that for the normalized flow in section 4 , but it does not include the rescaling by the characteristic length of space.

Since $\lambda<1 / 3$, we will confine our discussion to the case of non-negative cosmological constant, $\Lambda_{W} \geq 0$, so that the effective speed of light is real, and investigate the structure of the fixed points and their stability properties. The choices $\Lambda_{W}>0$ and $\Lambda_{W}=0$ will be discussed separately, although the latter can be obtained as limiting case of the former. Mathematically it is also interesting to consider the more general situation, without imposing any restrictions on $\lambda$ and $\Lambda_{W}$, but these cases will not be included here. ${ }^{11}$

\subsection{The axisymmetric Bianchi IX model}

The flow of the metric coefficients $\gamma_{i}(t)$ does not preserve the volume of $S^{3}$ in this case, and, therefore, the three coupled equations are rather difficult to investigate in all generality with $\gamma_{1} \neq \gamma_{2} \neq \gamma_{3}$. Restricting attention to axially symmetric configurations simplifies matters without shadowing too much the rich structure of the system. Our analysis will be based on the qualitative theory of dynamical systems, as in previous sections, but because of the mathematical complexity of the equations it is not possible to obtain explicit solutions in closed form, apart from the isotropic solution. Also, the reader should be aware of the limitations: the fixed points can become unstable in other directions, when axial symmetry is relaxed, and the conclusions drawn here may be altered and not be as general. Of course, this is part of a more general criticism for mini-superspace models compared to metric deformations with an infinite number of moduli taking place in the entire superspace.

With these explanations in mind, we are going to study the system of two equations

$$
\begin{aligned}
\frac{1}{\gamma_{1}} \frac{\mathrm{d} \gamma_{1}}{\mathrm{~d} \tau} & =-\frac{\sqrt{\gamma_{1}}}{\mu \gamma_{2}^{3}}\left(\gamma_{1}-\gamma_{2}\right)-\frac{8 \lambda-3}{4(3 \lambda-1)} \frac{\gamma_{1}}{\gamma_{2}^{2}}+\frac{2 \lambda-1}{3 \lambda-1} \frac{1}{\gamma_{2}}+\frac{\Lambda_{W}}{3 \lambda-1}, \\
\frac{1}{\gamma_{2}} \frac{\mathrm{d} \gamma_{2}}{\mathrm{~d} \tau} & =\frac{\sqrt{\gamma_{1}}}{2 \mu \gamma_{2}^{3}}\left(\gamma_{1}-\gamma_{2}\right)+\frac{4 \lambda-1}{4(3 \lambda-1)} \frac{\gamma_{1}}{\gamma_{2}^{2}}-\frac{\lambda}{3 \lambda-1} \frac{1}{\gamma_{2}}+\frac{\Lambda_{W}}{3 \lambda-1},
\end{aligned}
$$

\footnotetext{
${ }^{11}$ The nature of the corresponding fixed points changes drastically compared to the cases that are discussed in this paper. However, when $\Lambda_{W}<0$ it is more appropriate to consider Bianchi type VIII model geometries rather than Bianchi IX.
} 
which is obtained from (5.1) by setting $\gamma_{2}=\gamma_{3}$. The existence and properties of the fixed points depends crucially on the values of $\mu$, as in other examples considered so far. In particular, for $\mu<0$, an anisotropic fixed point will coexist with the isotropic one.

There is an exact solution of these equations which is available for all $\Lambda_{W} \geq 0$ and describes the evolution of the isotropic metric on $S^{3}$. This possibility does not arise for the normalized Ricci-Cotton flow, since the isotropic metric is a fixed point. In particular, setting all $\gamma_{i} \equiv \gamma$, we have the following result:

$$
\gamma(t)=A \exp \left(\frac{\Lambda_{W}}{3 \lambda-1} \tau\right)+\frac{1}{4 \Lambda_{W}} \text { for } \Lambda_{W}>0
$$

and

$$
\gamma(t)=-\frac{1}{4(3 \lambda-1)}\left(\tau-\tau_{0}\right) \text { for } \Lambda_{W}=0
$$

with $A$ and $\tau_{0}$ being arbitrary integration constant. Since $\lambda<1 / 3$, the metric flows to the isotropic fixed point (to be discussed next in detail for more general trajectories) as $\tau \rightarrow+\infty$.

The difference between $\Lambda_{W}>0$ and $\Lambda_{W}=0$ is reflected in the life-time of the solutions. When $\Lambda_{W}>0$ the solution has two branches: on the first branch $A>0$ and the solution is eternal existing for all $-\infty<\tau<+\infty$; it describes a round sphere with infinite radius in the infinite past flowing towards a round sphere with radius set by $\Lambda_{W}$ in the infinite future. The second branch corresponds to $A<0$ in which case the solution exists for $\tau_{\star} \leq t<+\infty$, with appropriately chosen $\tau_{\star}$, so that the sphere starts from zero radius and reaches the fixed point as $t \rightarrow+\infty$. On the other hand, when $\Lambda_{W}=0$, there is only one branch as the solution exists for $\tau_{0} \leq \tau<+\infty$, interpolating between a fully collapsed configuration at $\tau=\tau_{0}$ to a sphere of infinite radius in the infinite future.

\subsection{Classification of the fixed points}

First, we consider the case of non-vanishing cosmological constant and reserve the last subsection to study $\Lambda_{W}=0$ separately.

The isotropic fixed point. For $\Lambda_{W}>0$, there is a natural length scale in the problem that gives rise to the isotropic fixed point of the flow, irrespective of the sign of $\mu$,

$$
\gamma_{1}=\gamma_{2}=\gamma_{3}=\frac{1}{4 \Lambda_{W}} .
$$

This follows easily from the system of equations without assuming any restrictions on $\gamma_{1}$ and $\gamma_{2}$; it can also be shown that it is a fixed point of the more general system of equations with $\gamma_{1} \neq \gamma_{2} \neq \gamma_{3}$.

Linearizing around it as

$$
\gamma_{1}(t)=\frac{1}{4 \Lambda_{W}}(1+\delta x(t)), \quad \gamma_{2}(t)=\frac{1}{4 \Lambda_{W}}(1+\delta y(t)),
$$


we find that the small perturbations satisfy the characteristic matrix equation

$$
\frac{\mathrm{d}}{\mathrm{d} \tau}\left(\begin{array}{c}
\delta x \\
\delta y
\end{array}\right)=\Lambda_{W}\left(\begin{array}{cc}
-\frac{8}{\mu} \sqrt{\Lambda_{W}}-\frac{8 \lambda-3}{3 \lambda-1} & \frac{8}{\mu} \sqrt{\Lambda_{W}}+2 \frac{4 \lambda-1}{3 \lambda-1} \\
\frac{4}{\mu} \sqrt{\Lambda_{W}}+\frac{4 \lambda-1}{3 \lambda-1} & -\frac{4}{\mu} \sqrt{\Lambda_{W}}-2 \frac{2 \lambda-1}{3 \lambda-1}
\end{array}\right)\left(\begin{array}{l}
\delta x \\
\delta y
\end{array}\right) .
$$

The eigenvalues are

$$
\zeta_{1}=\frac{\Lambda_{W}}{3 \lambda-1}, \quad \zeta_{2}=-4 \Lambda_{W}\left(1+\frac{3}{\mu} \sqrt{\Lambda_{W}}\right)
$$

and so $\zeta_{1}<0$ whereas $\zeta_{2}$ can take all values, positive or negative, depending on $\mu$.

Keeping $\Lambda_{W}$ fixed and varying $\mu$ we obtain the following characterization of the isotropic fixed point:

- $\mu>0$ or $\mu<-3 \sqrt{\Lambda_{W}}$ : absolutely stable,

- $-3 \sqrt{\Lambda_{W}}<\mu<0$ : saddle point.

Note the emergence of a critical value, $\mu=-3 \sqrt{\Lambda_{W}}$, where the two eigenvalues $\zeta_{1}$ and $\zeta_{2}$ vanish, separating stability from instability along the corresponding eigen-directions. ${ }^{12}$ Also note for completeness that if we were allowing $\lambda>1 / 3$, the isotropic point would never be absolutely stable (it would be absolutely unstable or a saddle point in the respective intervals of the $\mu$-line.).

Actually, one can go further and investigate whether the exact isotropic running solution $\gamma(t)$, given by (5.5), is stable against small fluctuations,

$$
\gamma_{i}(t)=\gamma(t)+\delta \gamma_{i}(t)
$$

acting as attractor of nearby trajectories. Thus, given a small tube around the trajectory $\gamma(t)$, one is interested to know if any other trajectory with initial conditions inside this tube will remain there after some time and what is the size of tube that guarantees this attractor property. The differential equations for $\delta \gamma_{i}(t)$ are most conveniently stated using $\gamma(t)$ rather that $t$ as flow time. Then, within the axially symmetric ansatz (5.3), the linearized equations take the following form,

$$
\begin{aligned}
& \frac{\mathrm{d}}{\mathrm{d} \gamma(t)} \delta \gamma_{1}(t)=-\frac{2\left(\delta \gamma_{1}-\delta \gamma_{2}\right)}{\gamma\left(4 \Lambda_{W} \gamma-1\right)}\left[\frac{2(3 \lambda-1)}{\mu \sqrt{\gamma}}+4 \lambda-1\right]+\frac{4 \Lambda_{W} \delta \gamma_{1}}{4 \Lambda_{W} \gamma-1}, \\
& \frac{\mathrm{d}}{\mathrm{d} \gamma(t)} \delta \gamma_{2}(t)=\frac{\delta \gamma_{1}-\delta \gamma_{2}}{\gamma\left(4 \Lambda_{W} \gamma-1\right)}\left[\frac{2(3 \lambda-1)}{\mu \sqrt{\gamma}}+4 \lambda-1\right]+\frac{4 \Lambda_{W} \delta \gamma_{2}}{4 \Lambda_{W} \gamma-1},
\end{aligned}
$$

generalizing the characteristic matrix equations of small perturbations around the isotropic fixed point. These equations apply for all $\Lambda_{W}$ including the special case $\Lambda_{W}=0$ that will be discussed separately.

\footnotetext{
${ }^{12}$ The critical value of $\mu$ is similar to that found for the normalized Ricci-Cotton flow; direct comparison can be made by replacing $\sqrt{\Lambda_{W}}$ with $1 / L$ and rescaling $\mu$ with the characteristic length of space. In both cases, the critical value of $\mu$ occurs when the competing effects of the Ricci and Cotton deformations are balanced exactly.
} 
Solutions of this system can be expressed as functions of $t$ through $\gamma(t)$. Since $\delta \gamma_{i}$ are required to be small, for validity of the linearized analysis, the attractor property of the isotropic trajectory appears to be very limited. This behavior can be seen schematically in the phase portraits of the flow that will appear in the next two subsections.

Anisotropic fixed point. When $\mu<0$, there is an additional fixed point associated with the axially symmetric metric with coefficients

$$
\gamma_{1}=\frac{36 \mu^{2}}{\left(\mu^{2}+27 \Lambda_{W}\right)^{2}}, \quad \gamma_{2}=\gamma_{3}=\frac{9}{\mu^{2}+27 \Lambda_{W}} .
$$

There are no other restrictions on the values of $\mu$ for the existence of this second fixed point.

Notice that this new fixed point and the isotropic one will coalesce if $\mu=-3 \sqrt{\Lambda_{W}}$. For $-3 \sqrt{\Lambda_{W}}<\mu<0$, the anisotropic fixed point has $\gamma_{1}<\gamma_{2}$, whereas for $\mu<-3 \sqrt{\Lambda_{W}}$ it has $\gamma_{1}>\gamma_{2}$. Thus, $-3 \sqrt{\Lambda_{W}}$ appears as a critical value of $\mu$.

By considering small perturbations around the anisotropic fixed point, as

$$
\gamma_{1}(t)=\frac{36 \mu^{2}}{\left(\mu^{2}+27 \Lambda_{W}\right)^{2}}(1+\delta x(t)), \quad \gamma_{2}(t)=\frac{9}{\mu^{2}+27 \Lambda_{W}}(1+\delta y(t)),
$$

we find the characteristic matrix of the linearized system with respect to $\tau$

$$
\frac{1}{27(3 \lambda-1)}\left(\begin{array}{cc}
(9 \lambda-2) \mu^{2}-27 \Lambda_{W}(3 \lambda-1) & -(18 \lambda-5) \mu^{2}+27 \Lambda_{W}(6 \lambda-1) \\
-\frac{1}{2}(9 \lambda-5) \mu^{2}+\frac{27}{2} \Lambda_{W}(3 \lambda-1) & (9 \lambda-4) \mu^{2}-27 \Lambda_{W}(3 \lambda-2)
\end{array}\right) .
$$

The corresponding eigenvalues are

$$
\zeta_{ \pm}=\frac{1}{18(3 \lambda-1)}\left[2(3 \lambda-1) \mu^{2}-27 \Lambda_{W}(2 \lambda-1) \pm \sqrt{\Delta}\right],
$$

where

$$
\Delta=6(3 \lambda-1)(2 \lambda-1) \mu^{4}-72 \Lambda_{W} \mu^{2}(3 \lambda-1)^{2}+243 \Lambda_{W}^{2}\left(12 \lambda^{2}-6 \lambda+1\right) .
$$

The eigenvalues $\zeta_{ \pm}$are real. ${ }^{13}$ Since their product is given by

$$
\zeta_{+} \zeta_{-}=\frac{\left(\mu^{2}-9 \Lambda_{W}\right)\left(\mu^{2}+27 \Lambda_{W}\right)}{162(3 \lambda-1)}
$$

we note the appearance of a critical value $\mu$, which is the same as for the isotropic fixed point, $\mu=-3 \sqrt{\Lambda_{W}}$. Then, for $\mu<-3 \sqrt{\Lambda_{W}}$ the anisotropic point is saddle. On the other

\footnotetext{
${ }^{13}$ It follows by noting that the two roots of $\Delta$ occur at

$$
\mu_{ \pm}^{2}=\frac{3 \Lambda_{W}}{2(3 \lambda-1)(2 \lambda-1)}\left[4(3 \lambda-1)^{2} \pm \sqrt{2(3 \lambda-1)}\right]
$$

and they are complex for $\lambda<1 / 3$. Thus, $\Delta$ has the same sign as the coefficient of its $\mu^{4}$-term, which is positive.
} 
hand, in order to examine the stability of this fixed point for $-3 \sqrt{\Lambda_{W}}<\mu<0$, we consider the sum of the two eigenvalues,

$$
\zeta_{+}+\zeta_{-}=\frac{1}{9(3 \lambda-1)}\left[2(3 \lambda-1) \mu^{2}-27 \Lambda(2 \lambda-1)\right]
$$

which is now negative. Therefore, for $-3 \sqrt{\Lambda_{W}}<\mu<0$, the anisotropic fixed point is absolutely stable. Summarizing all results obtained above, we have the following:

- An isotropic fixed point exists for all $\mu$ and it is absolute stable when $\mu>0$ or $\mu<-3 \sqrt{\Lambda_{W}}$. For $-3 \sqrt{\Lambda_{W}}<\mu<0$ it is a saddle point.

- An anisotropic fixed point exists for all $\mu<0$. It is absolutely stable for $-3 \sqrt{\Lambda_{W}}<$ $\mu<0$ and saddle for $\mu<-3 \sqrt{\Lambda_{W}}$, which is reverse to the behavior of the isotropic fixed point.

As can be seen there are similarities as well as some differences with the classification of fixed points of the normalized Ricci-Cotton flow.

\subsection{Phase portraits of the flow}

A qualitative picture of the flow lines is provided by three consecutive phase portraits for different values of the parameter $\mu$. In all drawings we choose $\Lambda_{W}=0.25$ and $\lambda=0.1$, and so the three regimes are $\mu>0$ or $-1.5<\mu<0$ or $\mu<-1.5$. The isotropic fixed point appears at $\gamma_{1}=\gamma_{2}=1 / 4 \Lambda_{W}=1$.

First, we consider the case $\mu>0$ that exhibits only one (isotropic) fixed point, as shown in figure 11.

Next, we consider the case $\mu<0$ that exhibits an additional (anisotropic) fixed point and make the following choices for the plots shown in figures 12 and 13:

- $\mu=-1$ : the anisotropic fixed point occurs at $\gamma_{1} \simeq 0.60, \gamma_{2}=\gamma_{3} \simeq 1.16$.

- $\mu=-2$ : the anisotropic fixed point occurs at $\gamma_{1} \simeq 1.24, \gamma_{2}=\gamma_{3} \simeq 0.84$.

\subsection{The special case $\Lambda_{W}=0$}

Setting $\Lambda_{W}=0$ corresponds to taking the effective speed of light equal to zero, while keeping $\lambda<1 / 3$ arbitrary. It should be contrasted with the normalized Ricci-Cotton flow, which is independent of $\Lambda_{W}$ and has also zero effective speed of light.

The isotropic fixed point is now pushed to infinity and corresponds to a round $S^{3}$ with infinite radius. This is also apparent from the exact isotropic solution (5.6) that converges to it after infinitely long time. However, it is not strictly speaking a fixed point of the flow lines, since $\mathrm{d} \gamma_{1} / \mathrm{d} t$ and $\mathrm{d} \gamma_{2} / \mathrm{d} t$ do not vanish there when $\Lambda_{W}=0$.

When $\mu<0$ there is an anisotropic fixed point of the axially symmetric flow for

$$
\gamma_{1}=\frac{36}{\mu^{2}}=4 \gamma_{2}=4 \gamma_{3} \text {. }
$$




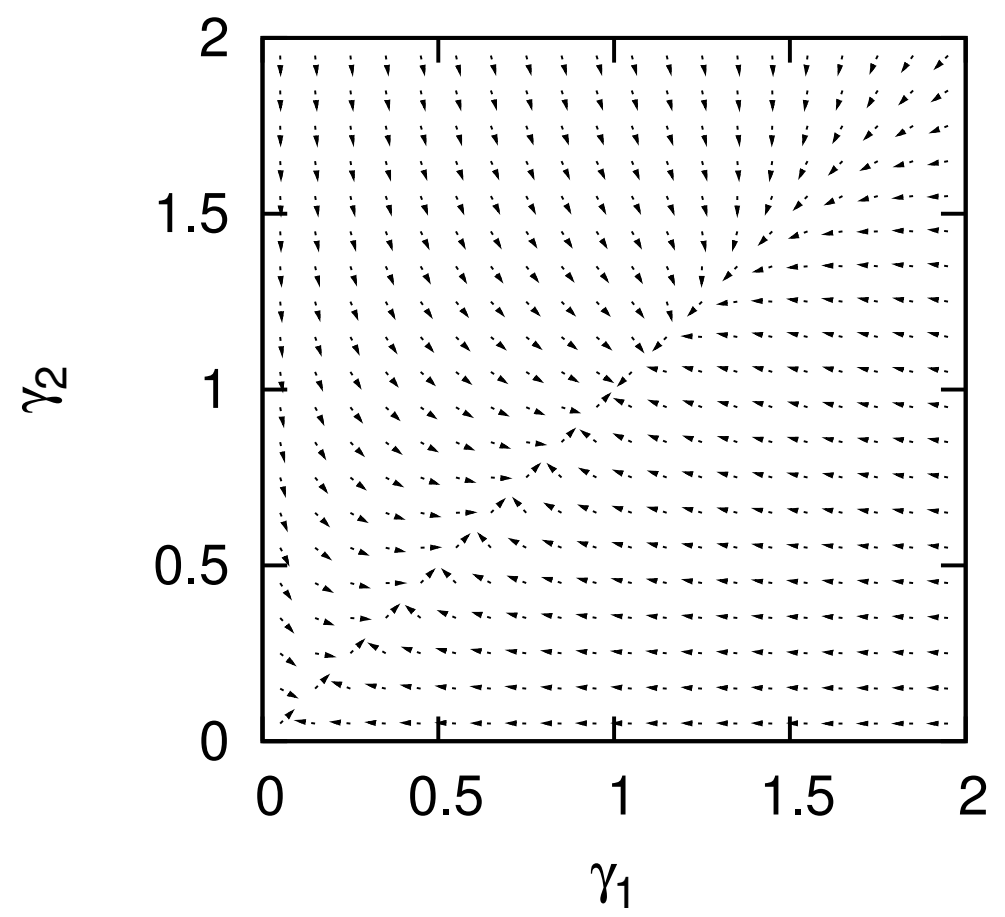

Figure 11. The flow lines for $\mu>0$; here, $\mu=1$.

This is always a saddle point because the corresponding eigenvalues of the characteristic matrix of small perturbations are real for $\lambda<1 / 3$ and their product is negative. The results follow setting $\Lambda_{W}=0$ in the expressions we had before (see e.g. (5.19)).

We include two phase portraits of the flow lines that are characteristic for $\mu>0$ and $\mu<0$, respectively, choosing $\lambda=0.1$. The case $\mu>0$ is shown first in figure 14 . Next, we consider the case $\mu<0$ that exhibits an additional (anisotropic) fixed point and make the choice $\mu=-5$. The anisotropic fixed point occurs at $\gamma_{1} \simeq 1.44, \gamma_{2}=\gamma_{3} \simeq 0.36$ as shown in figure 15.

Finally, concluding this section, we end up with an interesting observation that arose in our study of the flow lines. Along these lines, the volume of space changes, but the dependence on $t$ is not monotonic in general. It is therefore interesting to inquire in this context for the existence of bouncing solutions for which the volume reaches a minimum and then increases in time. Although this behavior is not generic, it seems to arise along particular flow lines that can be found by numerical scanning. An example of this kind is provided in figure 16 for an appropriate choice of initial data and couplings. Cases with full anisotropy, but with initial conditions close to axial symmetry, also seem to lead to bounces, including minima with very small volume. Similar conclusions hold when $\Lambda_{W}>0$, with or without axial symmetry, but we have not been able to obtain any quantitative characterization of the phenomenon so far. 


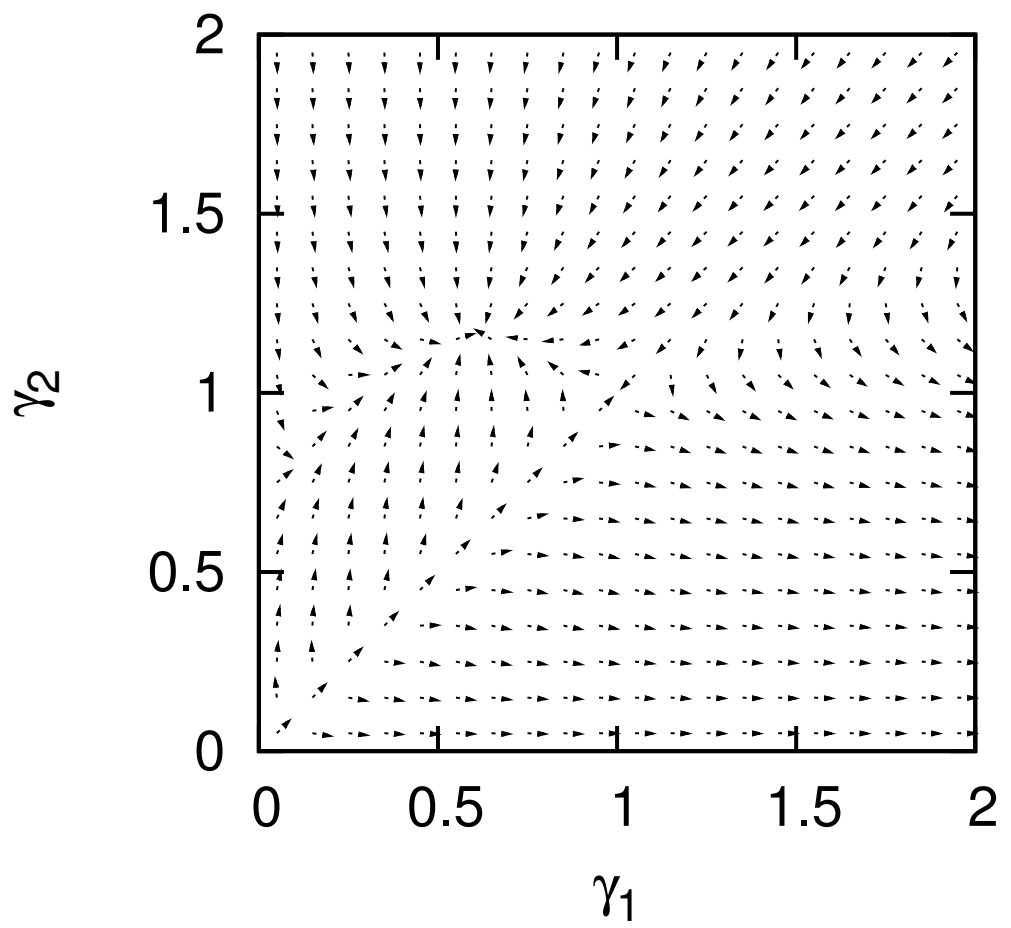

Figure 12. The flow lines for $-1.5=-3 \sqrt{\Lambda_{W}}<\mu<0$; here, $\mu=-1$.

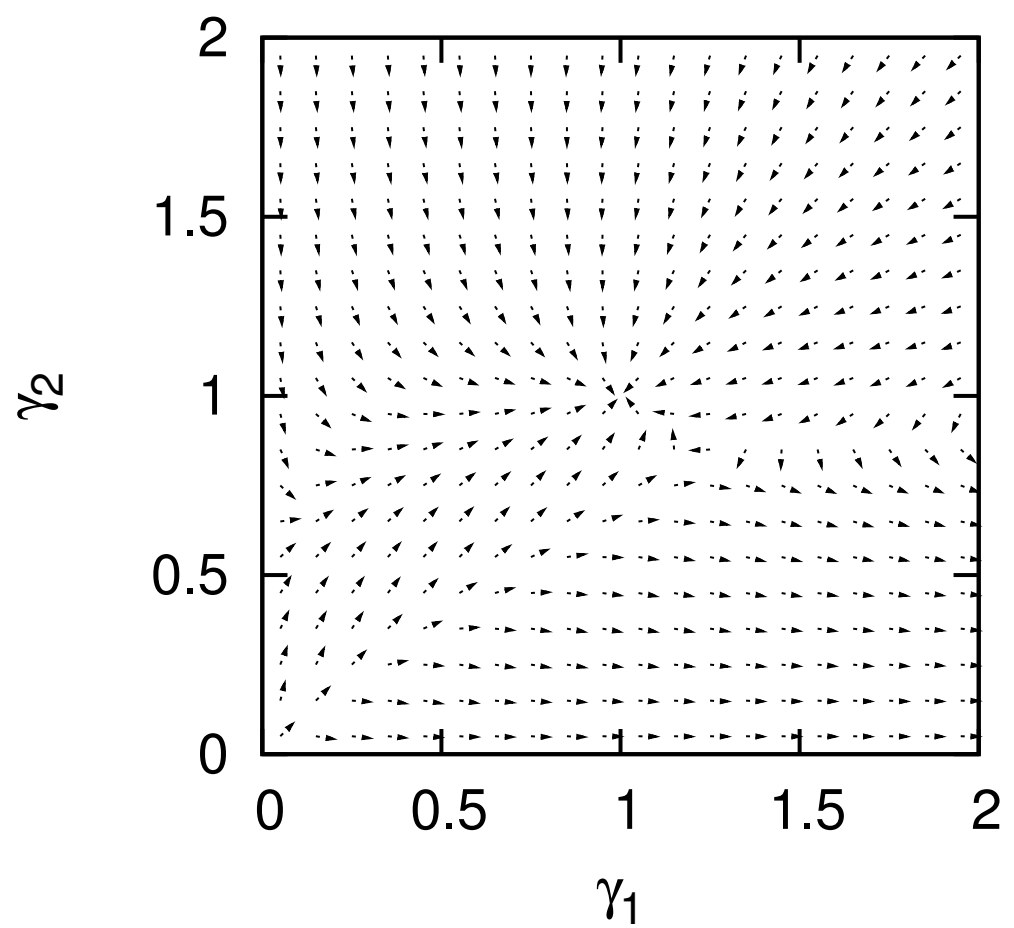

Figure 13. The flow lines for $\mu<-3 \sqrt{\Lambda_{W}}=-1.5$; here, $\mu=-2$. 


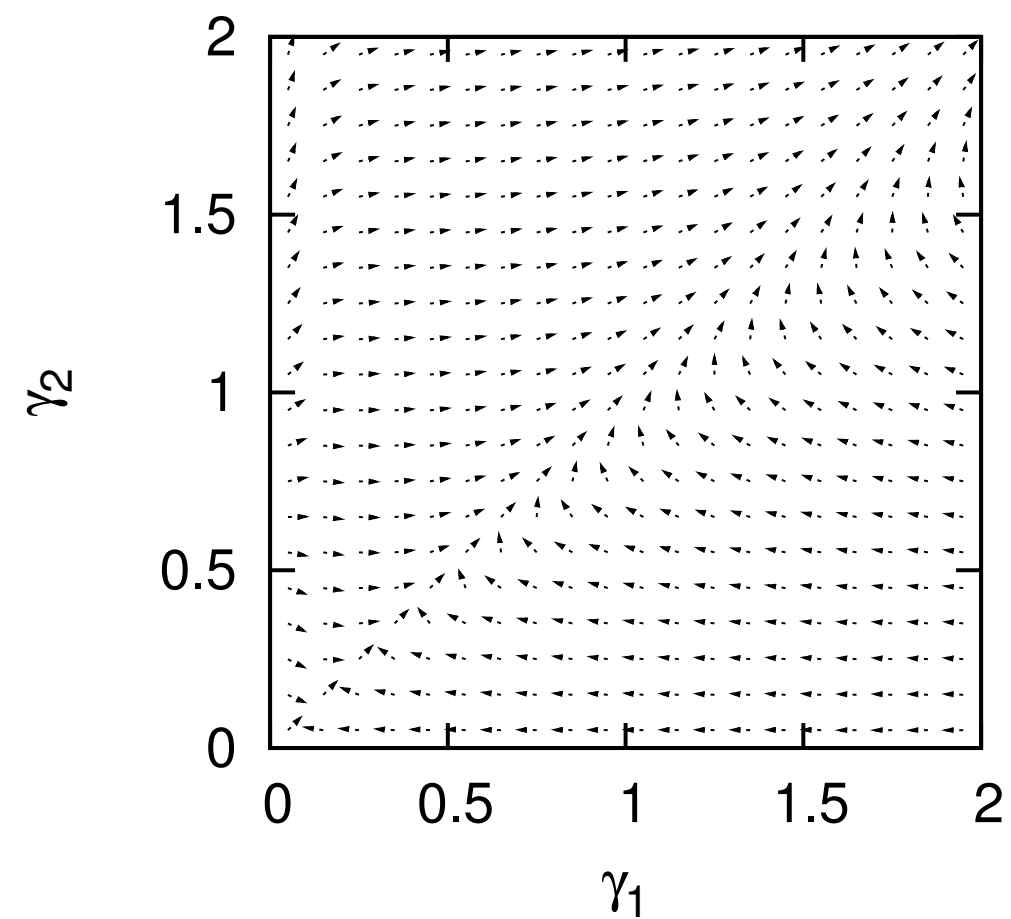

Figure 14. The flow lines for $\mu>0$; here, $\mu=1$.

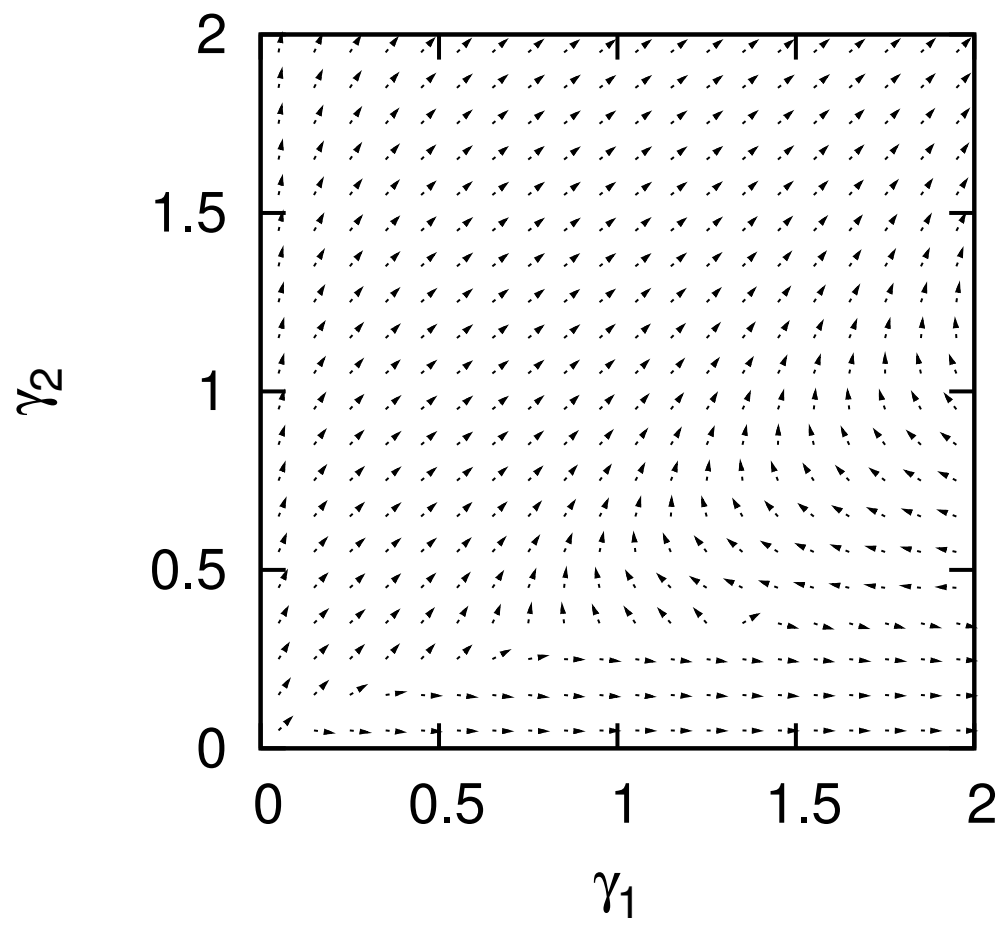

Figure 15. The flow lines for $\mu<0$; here, $\mu=-5$. 


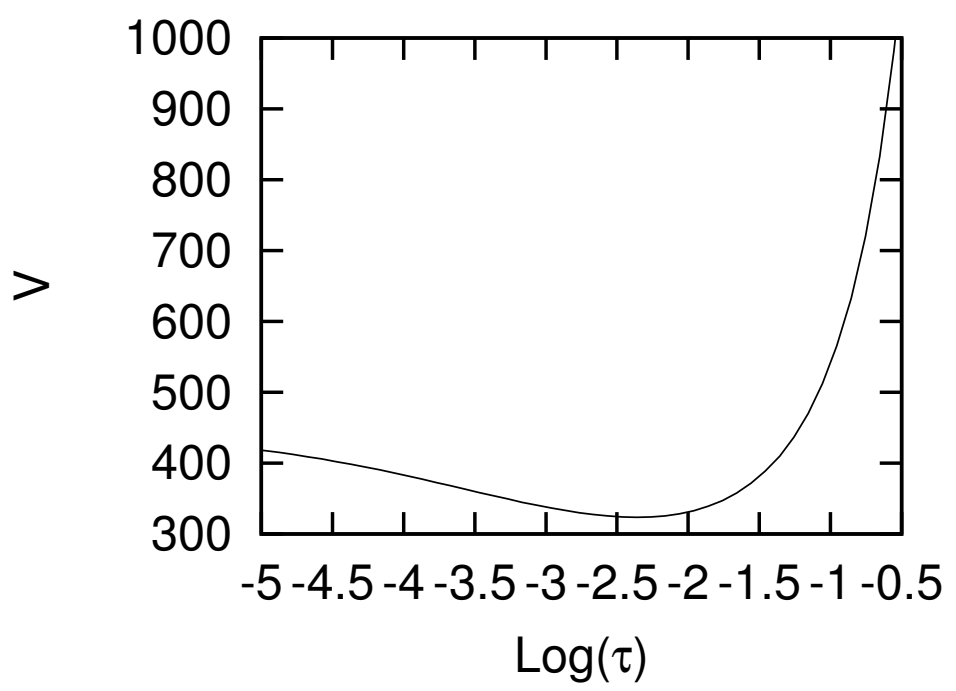

Figure 16. Bouncing solution with initial data $\gamma_{1}^{(0)}=28, \gamma_{2}^{(0)}=\gamma_{3}^{(0)}=4$ for $\lambda=0.26, \Lambda_{W}=0$ and $\mu=-5$.

The bouncing solutions can be regarded (in some sense) as the Euclidean space analogue of bouncing models in standard cosmology, which provide a viable alternative to inflation. Matter bounces have already appeared in studies of Hořava-Lifshitz cosmology (see, for instance, [56-58]), but they are also non-generic. It remains to be seen whether they have any special meaning and implications for the models we study here, although their occurrence does not require any matter couplings, as they are purely geometric, and, hence, different from those arising in cosmology.

\section{Space-time interpretation of the flow lines}

The solutions of geometric flows are shown as flow lines in the various phase portraits that have been drawn. Explicit solutions were obtained in special cases, whereas more general solutions can only be described pictorially. The problems that will be addressed in this section are the selection of flow lines that can qualify as regular gravitational-instanton solutions in four dimensions and the completeness of the corresponding space-time metrics. It will also be useful in this context to compare SU(2) instanton solutions of Hořava-Lifshitz theory with those of ordinary Einstein gravity.

According to the analysis of section 2.3, only the flow lines that interpolate between two fixed points (when more than one fixed point is present in our models) qualify as instantons. They are indeed finite-action solutions, and this property is sufficient to determine the global structure of space-time and its asymptotic behavior, and render the corresponding metrics complete. Our main result in a nut-shell is that all gravitational instantons with $\mathrm{SU}(2)$ isometry are globally $\mathbb{R} \times S^{3}$ describing a smooth deformation of $S^{3}$ as $t$ runs from $-\infty$ to $+\infty$, without ever encountering a singularity. The details are given below together with the complete classification of gravitational instantons with $\mathrm{SU}(2)$ isometry in Hořava- 
Lifshitz gravity with anisotropy parameter $z=3$. We will also compute their Euclidean action and determine their moduli spaces. In most cases we have explicit solutions. There are also a few other solutions that are shown to exist, but we have not (yet) been able to obtain expressions for their metric in closed form.

The variant of the theory with anisotropy parameter $z=2$ will not be especially discussed, since it is clear that it exhibits no instanton solutions (of the type we are considering here) with $\mathrm{SU}(2)$ isometry. Recall in this case that the relevant equations are provided by the Ricci flow on $S^{3}$, which takes the form (3.9) in proper time with arbitrary parameters $\lambda<1 / 3$ and $\Lambda_{W}>0$. Its fixed points are determined by the equations

$$
R_{i i}-\frac{2 \lambda-1}{2(3 \lambda-1)} R \gamma_{i}+\frac{\Lambda_{W}}{1-3 \lambda} \gamma_{i}=0, \quad i=1,2,3
$$

and clearly there is a unique solution given by the constant-curvature metric with $R=$ $6 \Lambda_{W}$. The absence of other fixed points, which is also implied on more general grounds by Poincaré's conjecture for $S^{3}$, shows that there can be no finite-action instanton solutions in this case.

Thus, in the following, we focus on instanton solutions of the $z=3$ theory and explain their properties, as outlined above.

\subsection{Global structure and completeness of the metrics}

First of all we examine the occurrence of singularities that can appear at finite proper time $t$ (or $\tau$, since the two are simply related by rescaling) and render the Euclidean spacetime manifold incomplete. Singularities arise when some or all of the metric coefficients of the Bianchi IX model geometry vanish and they are classified, in general, as nuts and bolts. Such singularities are intimately related to the fixed points of Killing vector fields by geometrical reasoning and they are independent of the gravitational equations. Following $[43,59]$ we recall that the structure of the fixed point set of a Killing vector field $\xi_{\mu}$ acting on any four-dimensional Riemannian manifold with metric $g_{\mu \nu}$ is determined by the rank of the $4 \times 4$ matrix $\nabla_{\mu} \xi_{\nu}$. This is an anti-symmetric matrix (since its symmetric part vanishes identically by definition of a Killing vector) which can have rank 4 or 2 ; rank 0 is excluded for, otherwise, the vector field vanishes everywhere. In the former case there are no directions left invariant at the tangent space of the fixed point, which, thus, appears to be isolated and it is called nut. In the latter case only a two-dimensional subspace of the tangent space at the fixed point remains invariant under the action of the Killing vector field, whereas the two-dimensional orthogonal complement rotates into itself. Then, the fixed point set is provided by this invariant two-dimensional subspace and it is naturally called bolt (it is typically a two-sphere, as in Bianchi IX geometries).

Nuts and bolts lead to incomplete manifolds, in general, but in certain cases the apparent singularities can be removed and provide regular and complete metrics with no curvature singularities. It all depends on the form of the metric as these singularities are approached. A removable nut singularity contributes one unit to the Euler number $\chi$ of the four-manifold and a removable $S^{2}$ bolt singularity contributes two units [59], following the theorems on fixed points. This counting applies to compact four-manifolds 
without boundary, but it also generalizes to non-compact spaces when the Killing vector field is either everywhere tangential to the boundary (as in space-times with homogeneous spatial sections that we are considering here) or is everywhere transverse. Thus, if $\chi \neq 0$, any Killing vector field will have at least one fixed point. No fixed points imply that $\chi=0$. Instanton solutions of Einstein and Hořava-Lifshitz gravity are quite different in this respect, as will be seen shortly, having important implications for their global topological structure.

Let us briefly review when such singularities can be removed from a Riemannian fourmanifold without referring to any specific theory or any solutions at the moment. Using locally the Bianchi IX form of the metric (3.16)

$$
\mathrm{d} s^{2}=\mathrm{d} t^{2}+a^{2}(t)\left(\sigma^{1}\right)^{2}+b^{2}(t)\left(\sigma^{2}\right)^{2}+c^{2}(t)\left(\sigma^{3}\right)^{2}
$$

we suppose that a singularity (nut or bolt) occurs at some finite proper distance, say $t=0$. It is well known that the metric has a removable nut singularity provided that near $t=0$ all metric coefficients vanish as

$$
a^{2}(t)=b^{2}(t)=c^{2}(t)=\frac{1}{4} t^{2} \quad \text { as } \quad t \rightarrow 0 .
$$

In this case we have a coordinate singularity of the polar coordinate system in $\mathbb{R}^{4}$ centered at $t=0$, which is simply removed by changing to a local Cartesian coordinate system near the point $t=0$ and adding it to the manifold. Also, it is well known that the metric has a removable bolt singularity provided that near $t=0$ two of the metric coefficients (say $a^{2}$ and $b^{2}$ ) become equal and the third vanishes as

$$
a^{2}(t)=b^{2}(t)=\text { finite }, \quad c^{2}(t)=\frac{1}{4} n^{2} t^{2} \quad \text { as } \quad t \rightarrow 0 \quad \text { with } \quad n \in \mathbb{Z} .
$$

Then, $a^{2}=b^{2}=R_{0}^{2}$ implies $a^{2}\left(\sigma^{1}\right)^{2}+b^{2}\left(\sigma^{2}\right)^{2}=R_{0}^{2}\left(\mathrm{~d} \vartheta^{2}+\sin ^{2} \vartheta \mathrm{d} \varphi^{2}\right)$, which is the canonical $S^{2}$ metric, while the $\mathrm{d} t^{2}+c^{2}\left(\sigma^{3}\right)^{2}$ part of the four-dimensional metric becomes $\mathrm{d} t^{2}+$ $\left(n^{2} t^{2} / 4\right) \mathrm{d} \psi^{2}$ near $t=0$, keeping $\vartheta$ and $\varphi$ constant. In this case, the topology of the manifold is locally $\mathbb{R}^{2} \times S^{2}$ and the $\mathbb{R}^{2}$ factor shrinks to a point on $S^{2}$ as $t \rightarrow 0$. By adjusting the range of $\psi$ so that $n \psi / 2$ runs from 0 to $2 \pi$, the apparent singularity at $t=0$ becomes a coordinate singularity of the polar system in $\mathbb{R}^{2}$ and can be removed as before. In all other cases the singularities cannot be removed and the manifold is incomplete.

The above reasoning is purely geometrical without reference to any field equations. Thus, different gravitational theories for Euclidean space-times of the form (6.2) may or may not lead to removable singularities at the fixed points of a Killing vector field. This depends on the way that the metric coefficients approach zero in the vicinity of a singularity and it is sensitive to the dynamics. The space-time singularities of Euclidean Einstein and Hořava-Lifshitz gravity (if they are present) will follow different power-law behavior, which, in turn, will affect the completeness of the corresponding metrics. Thus, the absence of nonremovable singularities provides a natural selection for the physically admissible solutions in those theories.

In Euclidean Hořava-Lifshitz gravity, a singularity can only arise if an eligible flow line reaches the boundaries — including the origin — of the physical parameter space, 
namely the two wedges of the first quadrant in the $(x, y)$ (or $\left.\left(\gamma_{1}, \gamma_{2}\right)\right)$ plane used in the phase portraits. Then, the flow becomes extinct as it cannot be continued beyond that point. Such singularities, if they are present, will arise at finite proper time (say $t=t_{0}$, but we can always set $t_{0}=0$ without loss of generality). This is obviously so because such singular points can also act as initial data for the time-reversed flow at a given finite instance of (proper) time. ${ }^{14}$ Such possibilities should be ruled out by the theory, unless the singularities are removable nuts or bolts, for, otherwise, the space-time metric will be singular. Using the Bianchi IX form of the metric (6.2) with $a^{2}=\gamma_{1}, b^{2}=\gamma_{2}$ and $c^{2}=\gamma_{3}$ (like in (3.1)), which is the appropriate choice in this case, we may set (as in nuts)

$$
\gamma_{1}=\beta_{1} t^{p_{1}}, \quad \gamma_{2}=\beta_{2} t^{p_{2}}, \quad \gamma_{3}=\beta_{3} t^{p_{3}}
$$

or alternatively (as in bolts)

$$
\gamma_{1}=\beta_{1}, \quad \gamma_{2}=\beta_{2}, \quad \gamma_{3}=\beta_{3} t^{p_{3}}
$$

and determine the allowed values of the coefficients $\beta_{i}$ and the exponents $p_{i}$ as $t \rightarrow 0$. In all cases we find that the first-order system of Bianchi IX equations for Hořava-Lifshitz gravity with general couplings does not lead to removable nuts or bolts. Only non-removable singularities can arise along the flow lines and they seem troublesome. ${ }^{15}$ Recall, however, that instanton solutions are rather restrictive, since they are described only by those trajectories that interpolate between two fixed points. Therefore, their metrics would be singular if any one of the fixed points were singular. But this is a contradiction of terms and cannot happen, since a fixed point, unlike a singularity, is only reached at infinite proper time, $t \rightarrow \pm \infty$ (with sign that depends on the direction of the flow), and not at at finite time. ${ }^{16}$ Thus, the instanton solutions protect themselves from the singularities that may otherwise arise by moving along generic flow lines. Whenever instanton solutions exist, their spaces will be always complete without any singularities.

In Euclidean Einstein gravity nuts and bolts are important elements in the theory of gravitational instantons, since all known solutions exist thanks to their presence. In this case, there is a certain class of gravitational instantons (6.2) that follows from the Ricci flow equations (3.11) in proper time $t$ with $\gamma_{1}=a, \gamma_{2}=b$ and $\gamma_{3}=c$, as explained in section 3.2. They include the trivial flat-space metric associated with the isotropic solution (3.12), having $a^{2}=b^{2}=c^{2}=t^{2} / 4$ everywhere (with $t_{0}=0$ ), as well as the Taub-NUT metric as the next non-trivial example with an additional axial symmetry $a(t)=b(t)$ and a removable nut singularity at the origin. ${ }^{17}$ The Atiyah-Hitchin metric provides an even

\footnotetext{
${ }^{14}$ Proving finiteness of extinction time for the solutions of geometric flow on certain three-manifolds is an intricate mathematical problem that will not be addressed in all generality, since we are only considering homogeneous geometries on $S^{3}$. It is a key point in Perelman's proof of the Poincaré conjecture based on Ricci flow [17-19] and it is not yet clear how it may generalize to the Ricci-Cotton flow.

${ }^{15}$ The power-law behavior of solutions as the flow lines approach the origin can also be read off from the exact solutions we have presented in various cases.

${ }^{16}$ This is also implied by the stability analysis around the fixed points, which shows that the time dependence of small fluctuations varies exponentially as $t$ goes to infinity.

${ }^{17}$ The gravitational field equations determine the Taub-NUT metric in the form shown in section 3.2,

$$
\frac{a(t)}{m}+\operatorname{arcsinh} \frac{a(t)}{m}=\frac{-t+t_{0}}{m}=\log \frac{2 m+c(t)}{2 m-c(t)}-2 m\left(\frac{1}{2 m+c(t)}-\frac{1}{2 m-c(t)}\right) .
$$
}


more complicated solution, which is fully anisotropic and complete [52-54]. It exhibits a removable bolt singularity at the origin, where $b=-c$ and it comes asymptotically close to Taub-NUT metric with $a=b$ as $t \rightarrow \infty$; we skip the details as they are not important for the present work. It turns out that these are the only complete gravitational instantons with $\mathrm{SU}(2)$ isometry that satisfy the Ricci flow equations (3.11); there is another complete metric with $\mathrm{SU}(2)$ isometry, the Eguchi-Hanson instanton, which has $a=b$ everywhere and a removable bolt singularity at the origin, but its coefficients satisfy a different system of first-order equations. Finally, we note for completeness, that exactly the same reasoning applies to gravitational instantons of Einstein gravity with cosmological constant [43, 59] that have removable nut and bolt singularities (e.g., $\mathbb{C} P^{2}$ as a gravitational instanton).

Thus, on the one hand, in Euclidean Einstein gravity the instanton spaces have nonvanishing Euler number and, in many cases, they also have non-vanishing signature (given roughly, but without any further explanation here, by the number of nuts minus the number of anti-nuts [59] that may be present). On the other hand, the instanton solutions of Hořava-Lifshitz gravity are globally $\mathbb{R} \times S^{3}$ having zero Euler number and signature. They simply describe the evolution of a three-sphere from $t=-\infty$ to $t=+\infty$ which deforms geometrically by the flow without ever becoming singular along the way; as such they resemble closer the behavior of ordinary instantons in particle theories rather than the instantons of Einstein gravity. This is not surprising in retrospect, since consistency of the Hořava-Lifshitz gravity is not questionable in the projectable case for space-times with global cross-product foliation structure. In either case, the corresponding metrics are regular everywhere and complete and their Euclidean gravitational action is finite though the reasoning is different for each theory. The finiteness of the action, which will be discussed more extensively shortly, makes these solutions mostly relevant in the quantum theory using, for instance, the path integral approach.

Let us also discuss the asymptotic structure of the solutions and compare them to those of ordinary gravity, since there are also important differences between the two theories. In Einstein gravity, the physical boundary conditions are largely determined by the positiveaction conjecture that requires that the action of any asymptotically Euclidean four-metric be positive, vanishing if and only if the space is flat $[43,44,60]$. Then, using the Bianchi IX form of the metric (6.2), the following possibilities arise at infinite proper distance $t$ : either there is a Euclidean infinity

$$
a^{2}(t)=b^{2}(t)=c^{2}(t)=\frac{1}{4} t^{2} \quad \text { as } \quad t \rightarrow \pm \infty
$$

when $0 \leq \psi \leq 4 \pi$ (it is a conical infinity when $0 \leq \psi \leq 2 \pi$ ) or a Taubian infinity

$$
a^{2}(t)=b^{2}(t)=t^{2}, \quad c^{2}=\text { finite } \quad \text { as } \quad t \rightarrow \pm \infty
$$

that encompasses the Taub-NUT metric. Combining all distinct boundary conditions that are available at $t=0$ and $t= \pm \infty$, one ends up with a few viable solutions that provide the list of all complete gravitational instanton metrics with $\mathrm{SU}(2)$ isometry. Similar considerations may apply to solutions with cosmological constant.

Setting $t_{0}=0$ for convenience, the power-law behavior of the coefficients close to the origin $t=0$ turns out to be $a^{2}(t)=b^{2}(t)=c^{2}(t)=t^{2} / 4$ and describes a removable nut singularity. 
In Horava-Lifshitz theory the situation is different. The Euclidean action is always positive-definite (at least for $\lambda<1 / 3$ that we are considering here) and vanishes when the three-dimensional metrics are vacua of topologically massive gravity without any time dependence. Thus, there are no a priori conditions on the asymptotic structure of instantons other than the mere existence of multiple vacua in three dimensions that serve as fixed points of the flow. Then, the asymptotic structure of space-time as $t \rightarrow \pm \infty$ is simply determined by the specific form of the metric coefficients $\gamma_{1}, \gamma_{2}$ and $\gamma_{3}$ at the initial and final fixed points, respectively. Their time dependence is exponential and it is completely determined by the eigenvalues of the characteristic matrix of small fluctuations around these fixed points. The departure from usual asymptotics (with zero or positive cosmological constant) is inherited to the solutions from the detailed balance condition and seems to be rather universal. It inflicts other classes of solutions, such as the construction of black-hole solutions whose right asymptotic structure requires departure from detailed balance using more general couplings [61-63] (otherwise there is no match with observations at large distances). This is also closely related to the problem of obtaining ordinary gravity by arguing (naively) that all higher-order curvature terms are suppressed in the infrared regime of the theory. ${ }^{18}$ However, it is not necessarily a big problem in the ultra-violet regime relevant to early time cosmology, where our discussion is applicable keeping $\lambda<1 / 3$. Abandoning detailed balance will ruin our general construction of instanton solutions.

\subsection{The action and moduli of SU(2) instanton metrics}

Let us now give some examples of instantons, based on the results described in previous sections, and compute their action $S_{\text {instanton }}=|\Delta W| / 2$ in each case separately (see (2.35)). In general, the superpotential consists of two terms $W=W_{\mathrm{CS}}+W_{\mathrm{EH}}$, which are given by the following expressions for Bianchi IX model geometries,

$$
W_{\mathrm{CS}}=\frac{16 \pi^{2}}{w_{\mathrm{CS}}}\left[1+\frac{1}{2 \gamma_{1} \gamma_{2} \gamma_{3}}\left(\gamma_{1}+\gamma_{2}-\gamma_{3}\right)\left(\gamma_{1}-\gamma_{2}+\gamma_{3}\right)\left(\gamma_{1}-\gamma_{2}-\gamma_{3}\right)\right]
$$

and

$$
W_{\mathrm{EH}}=-\frac{16 \pi^{2}}{\kappa_{W}^{2}}\left[\frac{1}{\sqrt{\gamma_{1} \gamma_{2} \gamma_{3}}}\left(\gamma_{1}^{2}+\gamma_{2}^{2}+\gamma_{3}^{2}-2 \gamma_{1} \gamma_{2}-2 \gamma_{2} \gamma_{3}-2 \gamma_{3} \gamma_{1}\right)+4 \Lambda_{W} \sqrt{\gamma_{1} \gamma_{2} \gamma_{3}}\right]
$$

and will be used next to evaluate the instanton action.

The cases below refer to instantons constructed from interpolating trajectories of the Ricci-Cotton flow for different couplings, changing as the complexity of the equations increases.

\footnotetext{
${ }^{18}$ For the same reason we cannot obtain the instantons of Einstein gravity from those of Hořava-Lifshitz theory. The first arise by dropping all higher-curvature terms and setting $\lambda=1$, whereas the latter exist in the full theory only for $\lambda<1 / 3$. Bianchi type IX models may offer a glimpse at this problem since the gravitational potential is derived from a superpotential in both cases (see, for instance, [44] for the derivation of the superpotential that governs $\mathrm{SU}(2)$ instantons in ordinary gravity; this reference also provides a neat qualitative picture for the completeness of their metrics using Hamiltonian methods). It should be easier to explore the renormalization of the coefficients of the superpotential and the parameter $\lambda$ for this class of mini-superspace models, as the theory is taken from the ultra-violet to the infrared domain.
} 
Cotton flow. For the pure Cotton flow treated in section 3.3 there are two fixed points: the isotropic point with $\gamma_{1}=\gamma_{2}=\gamma_{3}=L^{2} / 4$ and the anisotropic fixed point with $\gamma_{1}=$ $\gamma_{2}=x L^{2} / 4$, and $\gamma_{3}=L^{2} / 4 x^{2}$ that arises for $x=\infty$; two more anisotropic fixed points are obtained from it by permuting the three principal axes of $S^{3}$. The corresponding instanton, which is given in closed form by (3.28), describes the evolution of a fully squashed (flattened) sphere towards the round sphere as $t$ varies from $-\infty$ to $+\infty$; the anti-instanton follows by reversing the time direction.

Note that a natural entropy function associated with the volume-preserving deformation of $S^{3}$ (other than $W$ ) can be defined in this case. ${ }^{19}$ It is important for the mathematics of the Cotton flow, but, unlike $W$ that determines the action of the instanton, this entropy has no deeper meaning in space-time (as far as we can tell now). Also, there is no (yet) known analogue of it for the combined Ricci-Cotton flow.

The instanton solution has enhanced isometry $\mathrm{SU}(2) \times \mathrm{U}(1)$, since the deformation line possesses axial symmetry. Also, since the interpolating trajectory is unique, the instanton has no moduli other than the radius of the sphere at the fixed point. In this case, $W=W_{\mathrm{CS}}$ and one finds that the superpotential takes the following values at the two fixed points,

$$
W^{\text {iso }}=\frac{8 \pi^{2}}{w_{\mathrm{CS}}}, \quad W^{\text {aniso }}=\frac{16 \pi^{2}}{w_{\mathrm{CS}}} .
$$

Therefore, the action is

$$
S_{\text {instanton }}=\frac{4 \pi^{2}}{\left|w_{\mathrm{CS}}\right|}
$$

and it is independent of the modulus L. Obviously, there are no other instantons derived from the Cotton flow equations.

Normalized Ricci-Cotton flow. The normalized Ricci-Cotton flow has more than one fixed points when $\mu<0$, in which case there are instantons with $\mathrm{SU}(2)$ isometry interpolating between them as $t$ varies for $-\infty$ to $+\infty$.

Let us first consider the instantons connecting the two axially symmetric fixed points. Recall that the isotropic fixed point appears at $\gamma_{1}=\gamma_{2}=\gamma_{3}=L^{2} / 4$ and the anisotropic point at $\gamma_{1}=\gamma_{2}=L^{2} / 4 a$ and $\gamma_{3}=a^{2} L^{2} / 4$ (up to permutations of the axes of $S^{3}$ ), setting

\footnotetext{
${ }^{19}$ For Bianchi IX model geometries one can define in general an additional function (other than $W$ ) that changes monotonically under the Cotton flow. We consider

$$
F(t)=\frac{1}{\gamma_{1}^{2}}+\frac{1}{\gamma_{2}^{2}}+\frac{1}{\gamma_{3}^{2}}
$$

which is bounded from below by $3 /\left(\gamma_{1} \gamma_{2} \gamma_{3}\right)^{2 / 3}=3\left(16 \pi^{2} / V\right)^{4 / 3}$ for a three-sphere with volume $V$. The lower bound is attained in the fully isotropic case $\gamma_{1}=\gamma_{2}=\gamma_{3} . F(t)$ becomes infinite when the sphere is completely squashed in one or more directions; as such, it is a measure of the "shape entropy" of $S^{3}$. Using the Cotton flow (3.18), we obtain

$$
\frac{\mathrm{d} F}{\mathrm{~d} t}=-\frac{\kappa^{2}}{w_{\mathrm{CS}}\left(\gamma_{1} \gamma_{2} \gamma_{3}\right)^{3 / 2}}\left[\frac{\left(\gamma_{2}+\gamma_{3}\right)\left(\gamma_{2}-\gamma_{3}\right)^{2}}{\gamma_{1}^{2}}+\frac{\left(\gamma_{3}+\gamma_{1}\right)\left(\gamma_{3}-\gamma_{1}\right)^{2}}{\gamma_{2}^{2}}+\frac{\left(\gamma_{1}+\gamma_{2}\right)\left(\gamma_{1}-\gamma_{2}\right)^{2}}{\gamma_{3}^{2}}\right]
$$

and, therefore, $F(t)$ changes monotonically. For $w_{\mathrm{CS}}>0$, these properties of $F(t)$ suffice to prove the convergence of the flow lines to the fully isotropic fixed point regardless of initial conditions [46].
} 
for notational convenience

$$
a=\sqrt{-\frac{\mu}{3}}
$$

Then, explicit calculation shows that $W=W_{\mathrm{CS}}+W_{\mathrm{EH}}\left(\right.$ with $\left.\mu=w_{\mathrm{CS}} L / \kappa_{W}^{2}\right)$ takes the following form at the two fixed points,

$$
W^{\text {iso }}=\frac{8 \pi^{2}}{w_{\mathrm{CS}}}\left(1-9 a^{2}+3 a^{2} \Lambda_{W} L^{2}\right)
$$

and

$$
W^{\text {aniso }}=\frac{8 \pi^{2}}{w_{\mathrm{CS}}}\left(2+4 a^{6}-14 a^{3}+3 a^{2} \Lambda_{W} L^{2}\right) .
$$

Although $\Lambda_{W}$ does not appear in the normalized Ricci-Cotton flow equations, it enters into $W$ by contributing the same at all points (recall that the volume $V$ is preserved in this case). Consequently, the instanton action takes the value

$$
S_{\text {instanton }}=\frac{4 \pi^{2}}{\left|w_{\mathrm{CS}}\right|}(a-1)^{2}\left(4 a^{4}+8 a^{3}+12 a^{2}+2 a+1\right)
$$

and it is independent of $L$, as expected. Notice that it vanishes when $a=1(\mu=-3)$, as required, since the two fixed point coalesce and there is no instanton in this case. The action is non-zero and positive for all other values $\mu<0$.

The axisymmetric solutions of the normalized Ricci-Cotton flow have been constructed explicitly in section 4.4, but one should only use those branches that interpolate between the two fixed points. The stability analysis performed in section 4.2 shows that for $-3<$ $\mu<0$ the isotropic fixed point is absolutely unstable and the anisotropic is a saddle point. Therefore, there can be only one flow line interpolating between the two fixed points (corresponding to the axisymmetric solution we obtained) and the instanton has no moduli other than $L$. Exactly the same conclusion holds for $-6 \sqrt[3]{2}<\mu<-3$, since the isotropic fixed point is now absolutely stable and the anisotropic is a saddle point. The absence of moduli in these cases can also be seen schematically in figures 4 and 5 , respectively. Thus, for all $-6 \sqrt[3]{2}<\mu<0$ the instantons have enhanced $\mathrm{SU}(2) \times \mathrm{U}(1)$ isometry. The situation changes drastically when $\mu<-6 \sqrt[3]{2}$, since the isotropic fixed point is absolutely stable and the anisotropic is absolutely unstable. In this case, we have several flow lines interpolating between the two fixed points, as can also be seen schematically in figure 6 , and the instantons have an additional (real) modulus that labels these trajectories. The physical interpretation of this modulus is nothing else but the geometric shape of $S^{3}$ (there is only one shape modulus, since the volume of space is held fixed by specifying $L$ ). Of course, the instanton action is independent of all moduli.

The axisymmetric solution we have obtained in this case has enhanced $\mathrm{SU}(2) \times \mathrm{U}(1)$ isometry, whereas the other ones should only have an $\mathrm{SU}(2)$ isometry group. They all correspond to regular and complete metrics on $\mathbb{R} \times S^{3}$, but we have not been able to find them in closed form. They should be the analogue of the Atiyah-Hitchin metric for Hořava-Lifshitz gravity when $\lambda=-\infty$. Their explicit construction is an interesting open mathematical problem. 
Finally, we turn to instantons that owe their existence to the presence of totally anisotropic fixed points in the problem when $\mu<-6 \sqrt[3]{2}$. They have no moduli (other than their volume) since they connect a saddle point with a stable or an unstable fixed point. These instantons also have $\mathrm{SU}(2)$ isometry but no higher symmetry.

The value of the superpotential for the totally anisotropic fixed points (see also section 4.2) turns out to be

$$
W^{\text {total aniso }}=\frac{8 \pi^{2}}{w_{\mathrm{CS}}}\left(10+3 a^{2} \Lambda_{W} L^{2}\right) .
$$

Therefore, the instanton that interpolates between these points and the totally isotropic fixed point has action

$$
S_{\text {instanton }}=\frac{72 \pi^{2}}{\left|w_{\mathrm{CS}}\right|}\left(a^{2}+1\right) \text {. }
$$

It never becomes zero because these points cease to exist before they have the chance to meet with the isotropic point. Similarly, the instanton that interpolates between the totally anisotropic and the axially symmetric anisotropic fixed points has action

$$
S_{\text {instanton }}=\frac{16 \pi^{2}}{\left|w_{\mathrm{CS}}\right|}\left(a^{3}-4\right)\left(2 a^{3}+1\right) .
$$

The latter vanishes when $a^{3}=4(\mu=-6 \sqrt[3]{2})$, as the end-points coalesce in this case, and it is positive definite otherwise.

General Ricci-Cotton flow. The Ricci-Cotton flow with general couplings (provided that $\lambda<1 / 3$ and $\Lambda_{W}$ is non-negative) was found to exhibit two fixed points when $\mu<0$, in which case there are instanton solutions in Hořava-Lifshitz gravity. Recall that the isotropic point appears at $\gamma_{1}=\gamma_{2}=\gamma_{3}=1 / 4 \Lambda_{W}$ and the anisotropic point at $\gamma_{1}=$ $36 \mu^{2} /\left(\mu^{2}+27 \Lambda_{W}\right)^{2}$ and $\gamma_{2}=\gamma_{3}=9 /\left(\mu^{2}+27 \Lambda_{W}\right)$, assuming the presence of an axial symmetry $\gamma_{2}=\gamma_{3}$ for all time.

Taking $\mu<0$, we define, for notational convenience, the non-negative number

$$
a=-\frac{\mu}{3 \sqrt{\Lambda_{W}}}
$$

and evaluate the superpotential $W=W_{\mathrm{CS}}+W_{\mathrm{EH}}$ at the two fixed points. Using $\mu=$ $w_{\mathrm{CS}} / \kappa_{W}^{2}$, as defined in section 5 , we obtain the following results

$$
W^{\text {iso }}=\frac{8 \pi^{2}}{w_{\mathrm{CS}}}(1-6 a), \quad W^{\text {aniso }}=\frac{16 \pi^{2}\left(5 a^{4}-54 a^{2}+9\right)}{w_{\mathrm{CS}}\left(a^{2}+3\right)^{2}} .
$$

Therefore, the instanton action turns out to be

$$
S_{\text {instanton }}=\frac{12 \pi^{2}}{\left|w_{\mathrm{CS}}\right|} \frac{(a-1)^{2}}{\left(a^{2}+3\right)^{2}}\left(2 a^{3}+7 a^{2}+24 a+3\right) .
$$

Note that the action is manifestly positive-definite, as required, and vanishes when $a=1$ $\left(\mu=-3 \sqrt{\Lambda_{W}}\right)$, in which case the two fixed points coalesce and there is no instanton. 
Even in the presence of axial symmetry, which was used to simplify the analysis of the general Ricci-Cotton flow equations, we have not been able to obtain the interpolating solutions in closed form. Nevertheless, it is clear that a unique solution exists in this case, for all $\mu<0$, which interpolates between the two fixed points. For $0<a<1\left(-3 \sqrt{\Lambda_{W}}<\right.$ $\mu<0)$ the isotropic fixed point is saddle and the anisotropic is absolutely stable, and, therefore, there is a single flow line that connects the two. For $a>1\left(\mu<-3 \sqrt{\Lambda_{W}}\right)$ the isotropic fixed point is now absolutely stable and the anisotropic is a saddle point and, therefore, the interpolating flow line is again unique. This can also be seen by inspecting figures 12 and 13.

The solutions at hand have no moduli at all. ${ }^{20}$ They correspond to instantons with $\mathrm{SU}(2) \times \mathrm{U}(1)$ isometry. It will be very interesting to construct them explicitly. Also, other more general solutions with strict $\mathrm{SU}(2)$ isometry are expected to exist in the general case, with $\gamma_{1} \neq \gamma_{2} \neq \gamma_{3}$, but their investigation will not be pursued in the present work. We only note here that all anisotropic fixed points of the Ricci-Cotton flow equations with general couplings seem to be axially symmetric even when $\gamma_{1} \neq \gamma_{2} \neq \gamma_{3}$ at generic points. Thus, we expect to have instanton solutions without axial symmetry that interpolate between these fixed points, serving as the Hořava-Lifshitz analogue of the Atiyah-Hitchin metric. They should depend only on one free parameter.

The special case $\Lambda_{W}=\mathbf{0}$. Finally, note that as $\Lambda_{W}$ is taken to zero, while keeping $\mu$ fixed in the general system of Ricci-Cotton flow equations, $W^{\text {iso }}$ blows up to infinity, whereas $W^{\text {aniso }}$ remains finite, tending to the value $80 \pi^{2} / w_{\mathrm{CS}}$. Consequently, $S_{\text {instanton }}$ becomes infinite and one may consider it a problem, since instantons must have finite action. However, in this case, there is no contradiction, since the isotropic configuration ceases to be (strictly speaking) a fixed point when it is pushed away to infinity by setting $\Lambda_{W}=0$ and, therefore, the flow line that interpolates between the two points (see figure 15) does not qualify as instanton solution of the theory. In conclusion, there are no instanton solutions when $\Lambda_{W}=0$.

This completes our analysis of SU(2) gravitational instantons of Hořava-Lifshitz theory with anisotropy scaling parameter $z=3$. We have obtained complete classification of all explicit and implicit solutions that exist for all different couplings of the theory satisfying the detailed balance condition, provided that $\lambda<1 / 3$ and $\Lambda_{W}>0$. By the same token, the variant of the theory with scaling parameter $z=2$ does not exhibit any such instanton solutions. The results are on par with the classification of instantons with $\mathrm{SU}(2)$ isometry in Einstein gravity. The only missing technical part is the explicit construction of some of these instanton metrics.

We end this section with some general remarks concerning the existence and description of instanton metrics in Hořava-Lifshitz gravity without relying on isometry groups, such as $\mathrm{SU}(2)$. According to definition, they should be trajectories of the Ricci-Cotton flow equations interpolating between any two solutions of three-dimensional topologically

\footnotetext{
${ }^{20}$ Even the size of $S^{3}$ at the isotropic fixed point is not free, as $L$ was free to vary in the normalized flow, but it is determined by the parameters of the differential equations. Thus, it is not surprising that the instanton action depends on $\Lambda_{W}(\operatorname{through} a)$ in this case.
} 
massive gravity that provide the fixed points. The landscape of vacua of topologically massive gravity is not known completely ${ }^{21}$ and, therefore, it is difficult to make explicit general constructions. Also, it is rather difficult to investigate the general behavior of the flow equations by standard mathematical techniques, since they are third-order in space derivatives and even the short-time existence of solutions is difficult to establish in all generality. The formation and characterization of singularities is another related general open problem for these flow equations. Addressing these issues successfully will lead to further advances.

\section{Generalization to higher dimensions}

In this section we make a few remarks concerning higher-dimensional generalizations of Hořava-Lifshitz theory and the correspondence of its instanton solutions to the theory of higher-order geometric flows.

\subsection{Hořava-Lifshitz gravity in $4+1$ dimensions}

The general aspects have been reviewed in section 2 for all space-time dimensions. The theory is power-counting renormalizable when $z=D$ using the appropriate superpotential $W$. Let us concentrate on $D=4$ for definiteness, so that $W$ is the action of four-dimensional gravity with higher-order corrections of the general form [7]

$$
W[g]=\int \mathrm{d}^{4} x \sqrt{g}\left(\alpha C_{i j k \ell} C^{i j k \ell}+\beta R^{2}+\gamma\left(R-2 \Lambda_{W}\right)\right) .
$$

Here, $C_{i j k \ell}$ is the Weyl tensor and $R$ is the Ricci scalar curvature of a four-dimensional Riemannian metric $g$ that describes the geometry of spatial slices in a five-dimensional space-time with topology $\mathcal{M}_{5}=\mathbb{R} \times \mathcal{M}_{4}$. Here, there is no need to include the term $R_{i j} R^{i j}$ because it can be removed by a Gauss-Bonnet topological term, adjusting the coefficients $\alpha$ and $\beta$.

Thus, Hořava-Lifshitz gravity in $4+1$ dimensions with anisotropic scaling $z=4$ is defined by the action

$$
S=\frac{2}{\kappa^{2}} \int \mathrm{d} t \mathrm{~d}^{4} x \sqrt{g} N K_{i j} G^{i j k \ell} K_{k \ell}-\frac{\kappa^{2}}{8} \int \mathrm{d} t \mathrm{~d}^{4} x \sqrt{g} N\left(\frac{1}{\sqrt{g}} \frac{\delta W}{\delta g_{i j}}\right) \mathcal{G}_{i j k \ell}\left(\frac{1}{\sqrt{g}} \frac{\delta W}{\delta g_{k \ell}}\right)
$$

using the extrinsic curvature $K_{i j}$ of $\mathcal{M}_{4}$ and the metric $G^{i j k \ell}$ of superspace with parameter $\lambda$. Also, following the general discussion of section 2 , we will also take $\lambda<1 / 4$ so that the Euclidean counterpart of this action is manifestly bounded from below.

The theory with detailed balance is completely specified by the choice of $W[g]$. It is given by the general expression (7.1) in $D=4$; other appropriate choices of $W$ should be made in higher dimensions to render the theory power-counting renormalizable. We also

\footnotetext{
${ }^{21}$ Note, however, the recent work [64] that develops techniques to solve the field equations of topologically massive gravity (and other massive-gravity models) for three-dimensional geometries admitting a Killing vector field. Older results in this direction are neatly summarized in [65] although most of them focus on vacua with negative cosmological constant.
} 
note for completeness that if higher-order curvature functionals are chosen in $D$ spatial dimensions so that $z>D$, the resulting gravitational theory will be power-counting superenormalizable [7]. Such generalizations will not be considered at all in the present work.

Next, we illustrate the structure of the resulting equations by considering the simplest higher-dimensional case with $z=D=4$.

\subsection{Bach flow and its variants}

Solutions of the Euclidean five-dimensional Hořava-Lifshitz gravity can be obtained from the geometric-flow equation

$$
\frac{1}{N(t)} \partial_{t} g_{i j}= \pm \frac{\kappa^{2}}{2 \sqrt{g}} \mathcal{G}_{i j k \ell} \frac{\delta W[g]}{\delta g_{k \ell}}+\nabla_{i} \xi_{j}+\nabla_{j} \xi_{i}
$$

that describes deformations of the four-dimensional Riemannian metric $g_{i j}$. The lapse function $N(t)$ can be set equal to 1 by time redefinition.

The details can be worked out using the following identity, which is well known among people working in conformal Weyl gravity,

$$
B^{i j}=-\frac{1}{\sqrt{g}} \frac{\delta W_{\mathrm{Weyl}}}{\delta g_{i j}}
$$

where

$$
W_{\text {Weyl }}=\int \mathrm{d}^{4} x \sqrt{g} C_{i j k \ell} C^{i j k \ell}
$$

is the quadratic Weyl tensor action functional and

$$
B^{i j}=\nabla_{k} \nabla_{\ell} C^{i k j \ell}+\frac{1}{2} R_{k \ell} C^{i k j \ell}
$$

is the so called Bach tensor [66]. It is a fourth-order symmetric and traceless tensor that clearly vanishes when the four-dimensional metric is conformally flat. The Bach tensor provides the analogue of the Einstein tensor in the field equations of conformal Weyl gravity, and, as such, it is also covariantly conserved.

Thus, for this particular choice of superpotential $W$, the corresponding geometric flow takes the form

$$
\partial_{t} g_{i j}=\mp \frac{\kappa^{2}}{2} B_{i j}+\nabla_{i} \xi_{j}+\nabla_{j} \xi_{i}
$$

and it can be naturally called Bach flow. Its fixed points (modulo reparametrizations) are the vacuum solutions of conformal Weyl gravity and include the isotropic (constant curvature) metric on $S^{4}$. It is mathematically more interesting to pick the sign that drives the evolution towards the fixed points rather that away from them. Although this is a higher-order flow, it is better behaved mathematically than the third-order Cotton flow. Thus, one should investigate it in detail and attempt to construct solutions. It is a new geometric flow that has not appeared in the mathematics literature before, to the best of our knowledge. By restricting it to Kähler manifolds, it might be also interesting to compare it (and the variants which are discussed below) with other well known geometric flows of fourth-order, such as the Calabi flow [67]. 
If there is an additional contribution to $W$ given by the quadratic Ricci scalar curvature action,

$$
W_{R^{2}}=\int \mathrm{d}^{4} x \sqrt{g} R^{2}
$$

it will account for the gradient term

$$
H^{i j}=-\frac{1}{\sqrt{g}} \frac{\delta W_{R^{2}}}{\delta g_{i j}}
$$

with

$$
H^{i j}=2 g^{i j} \nabla_{k} \nabla^{k} R-2 \nabla^{i} \nabla^{j} R-2 R R^{i j}+\frac{1}{2} g^{i j} R^{2} .
$$

This tensor is symmetric but not traceless. Then, the complete flow equation will be a variant of the Bach flow receiving contributions from $B^{i j}$ and $H^{i j}$, which are both fourth-order. Of course, in the general case, there will also be subleading curvature terms associated with the Einstein tensor $G^{i j}$ by adding the four-dimensional Einstein-Hilbert action (possibly with a cosmological constant) to the superpotential $W$.

Instanton solutions will correspond to flow lines interpolating between different vacua of four-dimensional conformal Weyl gravity (and its deformations thereof), but again it seems rather difficult to derive explicit general results. Using four-dimensional model geometries may provide some simple and tractable mini-superspace models that are worth studying in the future.

Similar considerations apply to all higher-dimensional generalizations of Hor̆avaLifshitz gravity. In $D+1$ dimensions, the non-relativistic gravitational theory becomes power-counting renormalizable when the anisotropic scaling parameter is $z=D$. Then, for the appropriate choice of $W$, we obtain geometric flows of order $D$ that describe instantonlike configurations of the Euclidean $(D+1)$-dimensional theory when $\lambda<1 / D$. This framework hosts very naturally a whole hierarchy of geometric flows and provides a reason to study them.

\section{Conclusions and discussion}

We examined the Euclidean version of Hořava-Lifshitz gravity satisfying the detailed balance condition and described its instanton solutions as flow lines interpolating between different fixed points of a new class of geometric evolution equations, which are first-order in time. Although the specific couplings implied by detailed balance are rather restrictive (and sometimes appear to be problematic), the general connection between instanton solutions and geometric flows is rather interesting in many respects. Focusing to $3+1$ dimensions, where the potential term is derived from a superpotential $W$ given by the action functional of three-dimensional topologically massive gravity and the anisotropy scaling parameter of the theory is $z=3$, the driving curvature terms are provided by a certain combination of the Cotton and Ricci tensors as well as the cosmological constant term. The geometric-flow equations, called Ricci-Cotton flow, were shown to exhibit an entropy functional that is given by $W$ and can be used to put a lower bound on the Euclidean Hořava-Lifshitz gravitational action. 
Our construction requires $\lambda<1 / 3$ and $\Lambda_{W}>0$, but otherwise the parameters of the theory can be arbitrary within the class of detailed balance couplings. Fixed points of the flow are provided by classical solutions of the three-dimensional topologically massive gravity and they correspond to static solutions of the $(3+1)$-dimensional theory. As such, they include constant-curvature isotropic metrics in three dimensions as well as anisotropic configurations obtained by balancing the deformation effect of the Cotton and Ricci tensors. Since there is no general classification of these metrics, the landscape of fixed points remains largely unexplored to the best of our knowledge. Running solutions represent genuine time-dependent configurations, but they are even more difficult to investigate in exact terms. Thus, the Ricci-Cotton flow appears to be a rather complex system of equations that deserves proper mathematical study on general grounds. Addressing these problems in all generality remains out of reach at the moment, but some simple mini-superspace truncations of the equations help to obtain concrete results in simple cases that are interesting both physically and mathematically.

We found that the homogeneous model geometries on three-manifolds provide consistent truncation of the Ricci-Cotton flow equations. In particular, focusing on the Bianchi IX model geometries on $S^{3}$, so that the corresponding gravitational instantons exhibit $\mathrm{SU}(2)$ group of isometries, we were able to classify the fixed points of the flow (isotropic as well as anisotropic) and study their stability properties for a variety of different couplings. Some special solutions with axial symmetry (associated with $\mathrm{SU}(2) \times \mathrm{U}(1)$ isometry group) were constructed explicitly and their space-time interpretation was discussed in analogy with the gravitational instanton solutions of ordinary gravity. In particular, we have arrived at complete classification of the instanton solutions with $\mathrm{SU}(2)$ isometry. It remains to be seen whether more general running solutions can be constructed explicitly beyond their qualitative description based on the phase portraits of the flow. Also, it will be interesting to find other consistent reductions of the flow equations beyond the class of homogeneous geometries, but we have not yet been able to obtain any concrete results in this direction.

Another possibility that has not been discussed at all in this paper is to consider superrenormalizable versions of Hořava-Lifshitz gravity in $3+1$ dimensions with anisotropic scaling $z=4$. These are generated by a superpotential $W$ - other than the action functional of topologically massive gravity - which contains higher-order Ricci curvature terms such as $R_{i j} R^{i j}$ and $R^{2}$ on top of the cosmological Einstein-Hilbert action in three dimensions [7]. In this case, the instanton solutions will be described by geometric flows in three dimensions with fourth-order derivatives in their driving curvature terms. The resulting equations appear to have some nice mathematical properties (compared to the third-order Ricci-Cotton flow) and they also seem to admit consistent reduction to an autonomous system of ordinary differential equations for homogeneous model geometries. A particular choice of such $W$ is provided by the action of the so called "new massive gravity" in three dimensions that contains both terms $R_{i j} R^{i j}$ and $R^{2}$ with relative coefficient $-3 / 8$ [68]. We intend to investigate elsewhere the corresponding fourth-order flows [69], together with the associated instanton solutions, and examine the privileged role (if any) of new massive gravity in this context.

Higher-dimensional generalizations were also briefly discussed. It was pointed out that instanton solutions exist in all dimensions and their defining equations provide new classes 
of geometric-flow equations, such as the Bach flow in four dimensions. In general, the driving curvature terms of such flows contain spatial derivatives of order $z$ (equal to the anisotropy scale parameter that renders the higher-dimensional Hořava-Lifshitz gravity power-counting renormalizable) and they describe metric deformations on spatial slices of dimension $D=z$. The hierarchy of such flows has not been considered in the literature before and they certainly pose several interesting questions that are worth studying in the details. They should also be of interest to the mathematics community working on geometric analysis. In all cases, the non-relativistic theory of gravity provides a general framework to embed geometric evolution equations. The situation should be compared to general relativity and string theory, where such embedding is only possible in some very special cases, such as the Ricci flow on homogeneous three-geometries that can be interpreted as self-dual gravitational instantons in four dimensions or using some appropriately chosen higher-dimensional plane-wave gravitational backgrounds.

The off-shell formulation of string theory based on the world-sheet renormalization group equations provides a natural framework for the appearance of the Ricci flow (and other closely related geometric-flow equations) in gravitational physics. In this context, closed string tachyon condensation is described by transitions from one fixed point to another more stable fixed point. Thus, the lines of the Ricci flow resemble instanton transitions among different vacua of the string landscape. In Hořava-Lifshitz gravity, on the other hand, the Ricci flow (and its variants) describe the instantons of the theory when the anisotropic scaling is $z=2$. Therefore, it seems interesting to investigate further this aspect while searching for possible embedding of the non-relativistic theory of gravity into a more fundamental theory. Likewise, non-relativistic theories of gravity with higher anisotropic scaling, in particular $z=D$, and their instanton solutions may admit a similar description and interpretation in terms of a more fundamental theory. It is not yet known, however, whether the geometric evolution equations we are considering here can also arise as renormalization-group equations in a class of quantum field theories.

Finally, another interesting problem is the use of instantons for the quantization of Hořava-Lifshitz gravity. One possible line of work in this direction is the path integral approach over Euclidean space-times with applications to quantum cosmology in the spirit of Hartle-Hawking proposal. The quantization of mini-superspace models appears to be tractable, at least for homogeneous (but generally non-isotropic) geometries, and requires special attention. They can also provide some non-perturbative information about the quantum theory and a testing bed for comparison with the quantization of ordinary gravity.

It remains to be seen whether the non-relativistic theory of gravity is a viable alternative to Einstein gravity at very short distances. However, the simplified version of the theory with detailed balance can also play another role in physics, serving as landscape explorer of the vacuum structure of relativistic field theories determined by $W$ (with topologically massive gravity being just an example). It provides an effective particle model to describe transitions among different vacua through instantons. It also offers a dynamical principle for vacuum selection that is worth exploring further in all generality using the powerful tools of geometric flows and associated entropy functions. Advocating this point of view introduces a new twist to the subject and departs from the idea (and the problems 
that seem to accompany it) that Hořava-Lifshitz gravity is the ultra-violet completion of a fundamental theory. It could have also been used from the very beginning as an alternative motivation for the present work.

We hope to return to these topics elsewhere in the near future.

\section{Acknowledgments}

The authors would like to thank G. Huisken, A. Petkou, C. Sourdis and M. Taylor for stimulating discussions. Many of the ideas developed in the present work were triggered during the 2009 GGI workshop New Perspectives in String Theory. I. Bakas, F. Bourliot and M. Petropoulos would like to thank the LMU for kind hospitality. This research was supported by the Cluster of Excellence Origin and the Structure of the Universe in Munich, Germany, the French Agence Nationale pour la Recherche, contract 05-BLANNT09-573739 String Cosmology, the ERC Advanced Grant 226371 Mass Hierarchy and Particle Physics at the TeV Scale, the ITN programme PITN-GA-2009-237920 Unification in the LHC Era and the GRC APIC PICS-Grèce 3747.

Open Access. This article is distributed under the terms of the Creative Commons Attribution Noncommercial License which permits any noncommercial use, distribution, and reproduction in any medium, provided the original author(s) and source are credited.

\section{References}

[1] E.M. Lifshitz, On the theory of second order phase transitions I \& II, Zh. Eksp. Teor. Fiz. 11 (1941) $255 \& 269$.

[2] S. Chadha and H.B. Nielsen, Lorentz invariance as a low-energy phenomenon, Nucl. Phys. B 217 (1983) 125 [SPIRES].

[3] J. Iliopoulos, D.V. Nanopoulos and T.N. Tomaras, Infrared stability or anti grand unification, Phys. Lett. B 94 (1980) 141 [SPIRES].

[4] I. Antoniadis, J. Iliopoulos and T. Tomaras, On the infrared stability of gauge theories, Nucl. Phys. B 227 (1983) 447 [SPIRES].

[5] M. Petrini, Infrared stability of $N=4$ super Yang-Mills theory, Phys. Lett. B 404 (1997) 66 [hep-th/9704004] [SPIRES].

[6] P. Hořava, Membranes at Quantum Criticality, JHEP 03 (2009) 020 [arXiv:0812.4287] [SPIRES].

[7] P. Hořava, Quantum Gravity at a Lifshitz Point, Phys. Rev. D 79 (2009) 084008 [arXiv:0901.3775] [SPIRES].

[8] D. Orlando and S. Reffert, On the Renormalizability of Hor̆ava-Lifshitz-type Gravities, Class. Quant. Grav. 26 (2009) 155021 [arXiv:0905.0301] [SPIRES].

[9] F.-W. Shu and Y.-S. Wu, Stochastic Quantization of the Hor̆ava Gravity, arXiv:0906.1645 [SPIRES].

[10] C. Charmousis, G. Niz, A. Padilla and P.M. Saffin, Strong coupling in Hořava gravity, JHEP 08 (2009) 070 [arXiv:0905.2579] [SPIRES]. 
[11] M. Li and Y. Pang, A Trouble with Hořava-Lifshitz Gravity, JHEP 08 (2009) 015 [arXiv:0905.2751] [SPIRES].

[12] D. Blas, O. Pujolàs and S. Sibiryakov, On the Extra Mode and Inconsistency of Hor̆ava Gravity, JHEP 10 (2009) 029 [arXiv:0906.3046] [SPIRES].

[13] D. Blas, O. Pujolàs and S. Sibiryakov, A healthy extension of Hořava gravity, arXiv:0909.3525 [SPIRES].

[14] D. Blas, O. Pujolàs and S. Sibiryakov, Comment on 'Strong coupling in extended Hor̆ava-Lifshitz gravity', arXiv:0912.0550 [SPIRES].

[15] K. Koyama and F. Arroja, Pathological behaviour of the scalar graviton in Horaava-Lifshitz gravity, JHEP 03 (2010) 061 [arXiv:0910.1998] [SPIRES].

[16] R. Hamilton, Three-manifolds with positive Ricci curvature, J. Diff. Geom. 17 (1982) 255.

[17] G. Perelman, The entropy formula for the Ricci flow and its geometric applications, math/0211159 [SPIRES].

[18] G. Perelman, Ricci flow with surgery on three-manifolds, math/0303109 [SPIRES].

[19] G. Perelman, Finite extinction time for the solutions to the Ricci flow on certain three-manifolds, math/0307245 [SPIRES].

[20] H.-D. Cao, B. Chow, S.-C. Chu and S.-T. Yau eds., Collected Papers on Ricci Flow, Series in Geometry and Topology, 37, International Press, Somerville U.S.A. (2003).

[21] J.W. Morgan and G. Tian, Ricci Flow and the Poincaré Conjecture, Clay Mathematics Monographs, Amer. Math. Soc., Cambridge U.S.A. (2007) [math.DG/0607607].

[22] D. Friedan, Nonlinear Models in Two Epsilon Dimensions, Phys. Rev. Lett. 45 (1980) 1057 [SPIRES].

[23] D.H. Friedan, Nonlinear Models in Two + Epsilon Dimensions, Ann. Phys. 163 (1985) 318 [SPIRES].

[24] C. Schmidhuber and A.A. Tseytlin, On string cosmology and the $R G$ flow in $2-D$ field theory, Nucl. Phys. B 426 (1994) 187 [hep-th/9404180] [SPIRES].

[25] I. Bakas, D. Orlando and P.M. Petropoulos, Ricci flows and expansion in axion-dilaton cosmology, JHEP 01 (2007) 040 [hep-th/0610281] [SPIRES].

[26] M. Cvetič, G.W. Gibbons, H. Lü and C.N. Pope, Cohomogeneity one manifolds of Spin(7) and $G_{2}$ holonomy, Phys. Rev. D 65 (2002) 106004 [hep-th/0108245] [SPIRES].

[27] I. Bakas and K. Sfetsos, unpublished work (2004).

[28] G.W. Gibbons, private communication (2005).

[29] F. Bourliot, J. Estes, P.M. Petropoulos and P. Spindel, Gravitational instantons, self-duality and geometric flows, arXiv:0906.4558 [SPIRES].

[30] J.B. Hartle and S.W. Hawking, Wave function of the universe, Phys. Rev. D28 (1983) 2960 [SPIRES].

[31] C.W. Misner, Mixmaster universe, Phys. Rev. Lett. 22 (1969) 1071 [SPIRES].

[32] C.W. Misner, Quantum cosmology. 1, Phys. Rev. 186 (1969) 1319 [SPIRES].

[33] V.A. Belinsky, I.M. Khalatnikov and E.M. Lifshitz, Oscillatory approach to a singular point in the relativistic cosmology, Adv. Phys. 19 (1970) 525 [SPIRES]. 
[34] V.A. Belinsky, I.M. Khalatnikov and E.M. Lifshitz, A General Solution of the Einstein Equations with a Time Singularity, Adv. Phys. 31 (1982) 639 [SPIRES].

[35] J.D. Barrow, Chaotic behavior in general relativity, Phys. Rept. 85 (1982) 1 [SPIRES].

[36] I. Bakas, F. Bourliot, D. Lüst and M. Petropoulos, Mixmaster universe in Hořava-Lifshitz gravity, Class. Quant. Grav. 27 (2010) 045013 [arXiv:0911.2665] [SPIRES].

[37] Y.S. Myung, Y.-W. Kim, W.-S. Son and Y.-J. Park, Chaotic universe in the $z=2$ Hovava-Lifshitz gravity, arXiv:0911.2525 [SPIRES].

[38] L. Carroll, Alice's Adventures in Wonderland, MacMillan, London U.K. (1865).

[39] C.W. Misner, K.S. Thorne and J.A. Wheeler, Gravitation, Freeman, San Francisco U.S.A. (1973).

[40] M. Henneaux, A. Kleinschmidt and G.L. Gomez, A dynamical inconsistency of Hor̆ava gravity, Phys. Rev. D 81 (2010) 064002 [arXiv:0912.0399] [SPIRES].

[41] S. Deser, R. Jackiw and S. Templeton, Topologically massive gauge theories, Ann. Phys. 140 (1982) 372 [Erratum ibid. 185 (1988) 406] [SPIRES].

[42] S. Deser, R. Jackiw and S. Templeton, Three-Dimensional Massive Gauge Theories, Phys. Rev. Lett. 48 (1982) 975 [SPIRES].

[43] T. Eguchi, P.B. Gilkey and A.J. Hanson, Gravitation, Gauge Theories and Differential Geometry, Phys. Rept. 66 (1980) 213 [SPIRES].

[44] G.W. Gibbons and C.N. Pope, The Positive Action Conjecture and Asymptotically Euclidean Metrics in Quantum Gravity, Commun. Math. Phys. 66 (1979) 267 [SPIRES].

[45] R.S. Hamilton, The Ricci flow on surfaces, in Mathematics and General Relativity, Contemp. Math. 71, Amer. Math. Soc., Providence U.S.A. (1988), pg. 237-262.

[46] A.U.O. Kisisel, O. Sarioglu and B. Tekin, Cotton flow, Class. Quant. Grav. 25 (2008) 165019 [arXiv: 0803.1603] [SPIRES].

[47] J. Isenberg and M. Jackson, Ricci flow of locally homogeneous geometries on closed manifolds, J. Diff. Geom. 35 (1992) 723.

[48] G. Darboux, Mémoire sur la théorie des coordonnées curvilignes et des systèmes orthogonaux, Ann. Ec. Normale Supér. 7 (1878) 101.

[49] G.-H. Halphen, Sur un système d'équations différentielles, C.R. Acad. Sc. Paris 92 (1881) 1001.

[50] G.-H. Halphen, Sur certains systèmes d'équations différentielles, C.R. Acad. Sc. Paris 92 (1881) 1004.

[51] L.A. Takhtajan, A Simple example of modular forms as tau functions for integrable equations, Theor. Math. Phys. 93 (1992) 1308 [SPIRES].

[52] M.F. Atiyah and N.J. Hitchin, Low-Energy Scattering of Nonabelian Monopoles, Phys. Lett. A 107 (1985) 21 [SPIRES].

[53] M.F. Atiyah and N.J. Hitchin, The Geometry and Dynamics of Magnetic Monopoles, Porter Lectures, Princeton University Press, Princeton U.S.A. (1988).

[54] G.W. Gibbons and N.S. Manton, Classical and Quantum Dynamics of BPS Monopoles, Nucl. Phys. B 274 (1986) 183 [SPIRES]. 
[55] Y. Nutku and P. Bakler, Homogeneous, anisotropic three manifolds of topologically massive gravity, Annals Phys. 195 (1989) 16 [SPIRES].

[56] G. Calcagni, Cosmology of the Lifshitz universe, JHEP 09 (2009) 112 [arXiv:0904.0829] [SPIRES].

[57] E. Kiritsis and G. Kofinas, Hořava-Lifshitz Cosmology, Nucl. Phys. B 821 (2009) 467 [arXiv: 0904.1334] [SPIRES].

[58] R. Brandenberger, Matter Bounce in Hor̆ava-Lifshitz Cosmology, Phys. Rev. D 80 (2009) 043516 [arXiv: 0904.2835] [SPIRES].

[59] G.W. Gibbons and S.W. Hawking, Classification of Gravitational Instanton Symmetries, Commun. Math. Phys. 66 (1979) 291 [SPIRES].

[60] G.W. Gibbons, S.W. Hawking and M.J. Perry, Path Integrals and the Indefiniteness of the Gravitational Action, Nucl. Phys. B 138 (1978) 141 [SPIRES].

[61] A. Kehagias and K. Sfetsos, The black hole and FRW geometries of non-relativistic gravity, Phys. Lett. B 678 (2009) 123 [arXiv: 0905.0477] [SPIRES].

[62] H. Lü, J. Mei and C.N. Pope, Solutions to Hořava Gravity, Phys. Rev. Lett. 103 (2009) 091301 [arXiv: 0904.1595] [SPIRES].

[63] E. Kiritsis and G. Kofinas, On Hořava-Lifshitz 'Black Holes', JHEP 01 (2010) 122 [arXiv:0910.5487] [SPIRES].

[64] M. Gurses, Killing Vector Fields in Three Dimensions: A Method to Solve Massive Gravity Field Equations, arXiv:1001.1039 [SPIRES].

[65] D.D.K. Chow, C.N. Pope and E. Sezgin, Classification of solutions in topologically massive gravity, arXiv:0906.3559 [SPIRES].

[66] R. Bach, Zur Weylschen Relativitätstheorie und der Weylschen Erweiterung des Krümmungsbegriffs, Math. Zeitschr. 9 (1921) 110.

[67] E. Calabi, Extremal Kähler metric, in Seminar on Differential Geometry, S.-T. Yau ed., Annals of Mathematical Studies 102, Princeton University Press, Princeton U.S.A (1982).

[68] E.A. Bergshoeff, O. Hohm and P.K. Townsend, Massive Gravity in Three Dimensions, Phys. Rev. Lett. 102 (2009) 201301 [arXiv:0901.1766] [SPIRES].

[69] I. Bakas and C. Sourdis, work in progress. 